\title{
WestVirginiaUniversity
}

THE RESEARCH REPOSITORY @ WVU

Graduate Theses, Dissertations, and Problem Reports

2013

\section{Reproducibility of Retention Indices Examining Column Type}

Amanda M. Cadau

West Virginia University

Follow this and additional works at: https://researchrepository.wvu.edu/etd

\section{Recommended Citation}

Cadau, Amanda M., "Reproducibility of Retention Indices Examining Column Type" (2013). Graduate Theses, Dissertations, and Problem Reports. 440.

https://researchrepository.wvu.edu/etd/440

This Thesis is protected by copyright and/or related rights. It has been brought to you by the The Research Repository @ WVU with permission from the rights-holder(s). You are free to use this Thesis in any way that is permitted by the copyright and related rights legislation that applies to your use. For other uses you must obtain permission from the rights-holder(s) directly, unless additional rights are indicated by a Creative Commons license in the record and/ or on the work itself. This Thesis has been accepted for inclusion in WVU Graduate Theses, Dissertations, and Problem Reports collection by an authorized administrator of The Research Repository @ WVU. For more information, please contact researchrepository@mail.wvu.edu. 


\title{
Reproducibility of Retention Indices Examining Column Type
}

\author{
Amanda M. Cadau \\ Thesis submitted to the \\ Eberly College of Arts and Sciences \\ at West Virginia University \\ in partial fulfillment of the requirements \\ for the degree of \\ Master of Science \\ in \\ Forensic and Investigative Science \\ Suzanne Bell, Ph.D., Chair \\ Glen Jackson, Ph.D. \\ Keith Morris, Ph.D.
}

Department of Forensic and Investigative Science

Morgantown, West Virginia

2013

Keywords: Retention Index, Kovats Retention Index, GC/MS, Drugs of Abuse 


\title{
ABSTRACT \\ Reproducibility of Retention Indices Examining Column Type
}

\begin{abstract}
Amanda M. Cadau
Requests for drug analysis accounted for nearly half of all requests (DNA, toxicology, latent prints, etc.) submitted to forensic laboratories in the United States (Storm et al., 2010). A study by RTI International found that laboratories are only capable of processing about $80 \%$ of their controlled substances requests. Based upon this statistic, an estimated 220,000 cases per year would be back-logged. Therefore, tools that can improve the process of seized drug analysis would be of use. This project addresses one such concept, the use of retention indices as a means to improve drug screening using gas chromatography/mass spectrometry (GC/MS).

When drugs that co-elute (such as methamphetamine and phentermine) are analyzed, a mass spectrum would not be useful as both substances will give similar spectra. This presents problems especially with designer drugs that are similar in structure and molecular weight. Retention index, when coupled with mass spectrometry, can differentiate drugs that co-elute, thus reducing false positives and improving screening processes and the reliability of identification of compounds with similar mass spectra.

To date, retention indices have been listed as single whole numbers by providers such as the DEA and NIST. A survey of data obtained from different sources show that is often in disagreement between sources for the same compound on the same column. This is not unexpected, but does indicate the need for determination of a realistic range (i.e., uncertainty) that should accompany any reported retention index. In the case of similar compounds, this is not just desirable, it is essential. The goal of this project was to examine factors that could contribute to uncertainty (different instruments, different days, etc.), evaluate the relative importance of these factors, and to suggest defensible methods for incorporating uncertainty into retention index values.

This study used a DB-5 equivalent column (5\% phenyl 95\% methylpolysiloxane stationary phase) to find the uncertainty range between two different manufactures of gas chromatographs and manufacturers of columns. It was determined that a thinner film afforded the best repeatability of RI within the same instrument when concentrations were in the parts-per-million range. It was also determined that with the same experimental parameters and stationary phase but varying film thickness, the RI values could not be combined between instruments. The RI values from the thicker film produced a slightly larger uncertainty range compared to the thinner film. However, RI could be used to identify the components of a mixture that co-elute with poor resolution of peaks. RI has the potential to reduce false positives and decrease the backlog of drug analysis requests in forensic laboratories. However, for a universal library, the experimental parameters would have to be standardized on the most common columns used in drug analysis (DB-1 and DB-5 equivalents).
\end{abstract}




\section{Acknowledgements}

I would like to acknowledge:

Dr. Suzanne Bell, for her mentor ship, support, and advice for this project and throughout my college career at West Virginia University.

My committee members: Dr. Keith Morris, and Dr. Glen Jackson for the review and assistance with this work.

Mr. Scott Vajdos of Harris County Institute of Forensic Sciences, for his assistance in the beginning phases of this study and for the use of data compiled for SWGDRUG (complied by Mr. Vajdos and Ms. Angeline Yap Tiong Whei of SWGDRUG).

The Oklahoma State Bureau of Investigation (OSBI) and Kevin Kramer for the use of the macro used for the calculation of Retention Indices.

I would like to thank Holly McCall and Corey Nida for their assistance and support throughout this study. It was truly invaluable and much appreciated. 


\section{Table of Contents}

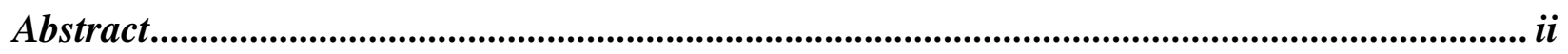

Acknowledgements........................................................................................................

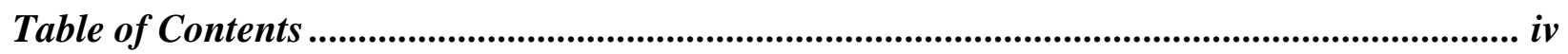

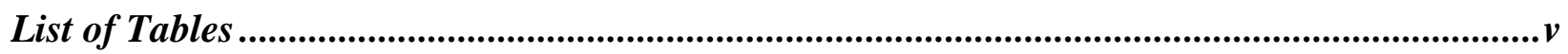

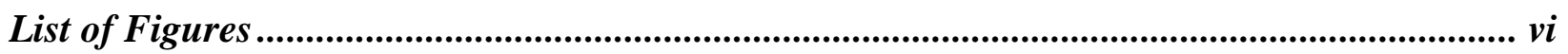

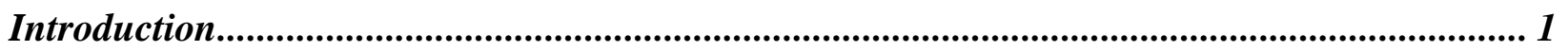

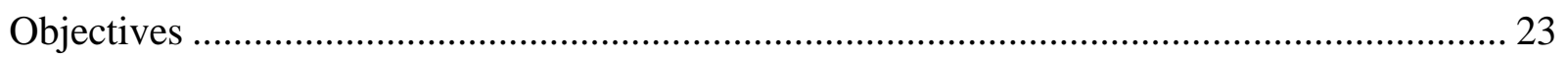

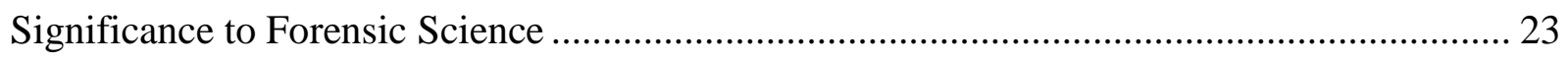

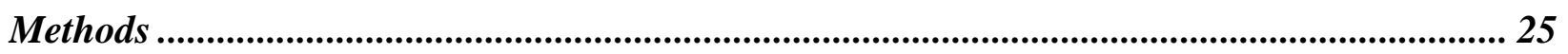

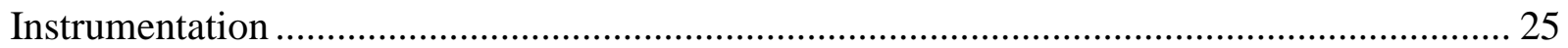

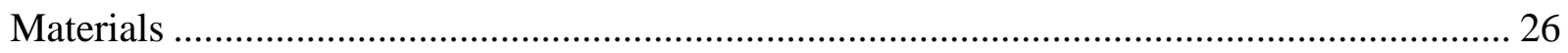

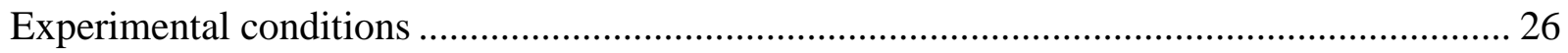

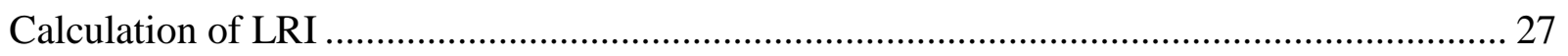

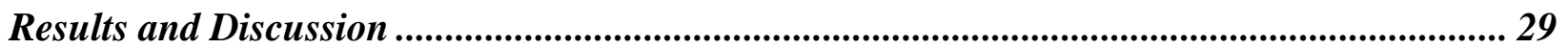

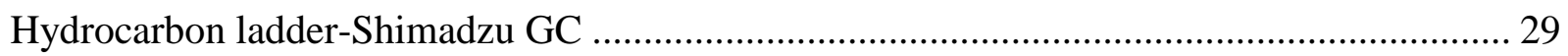

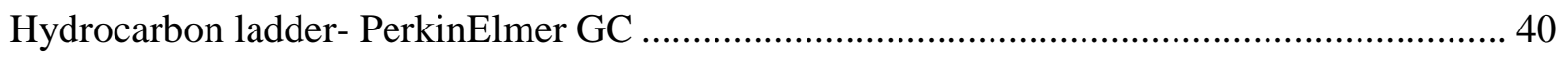

Hydrocarbon ladder-PerkinElmer GC (modified temperature program from Method 3) ........ 49

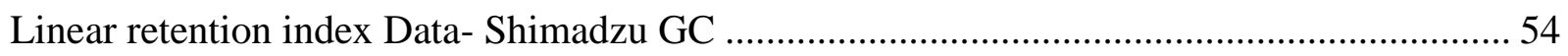

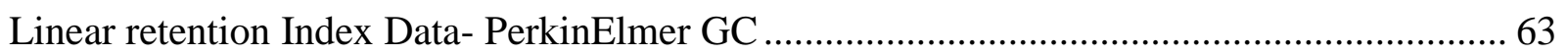

Combined LRI data from the Shimadzu GC and the PerkinElmer GC .................................. 71

Resolution study for methamphetamine and phentermine .................................................... 72

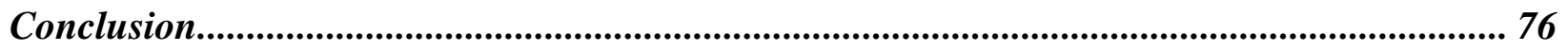

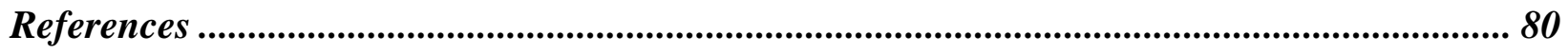

Appendix A: Shimadzu GC Data............................................................................................. 82

Appendix B: PerkinElmer GC Data............................................................................................... 91 


\section{List of Tables}

Table 1. RI values of 9 illicit drugs over four stationary phases from SWGDRUG. ................. 15

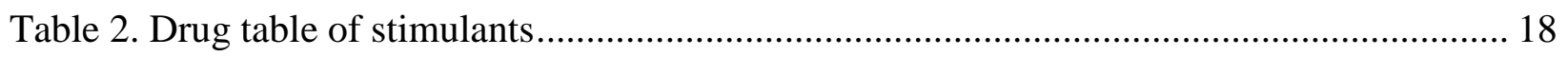

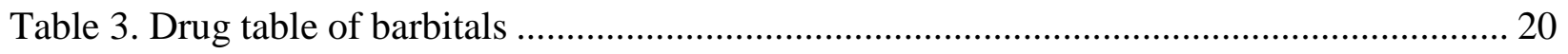

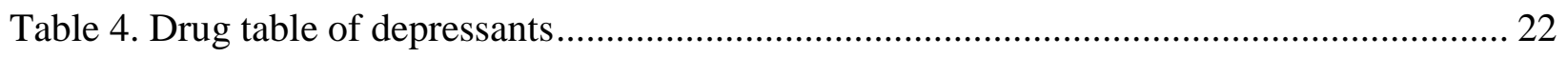

Table 5. Parameters of instruments..................................................................................... 25

Table 6.Intra-day variability of retention times of 5 replicates of hydrocarbon ladder ............... 32

Table 7. Intra-day variability of retention times of 10 replicates of the hydrocarbon ladder ...... 36

Table 8. Inter-day variability of hydrocarbon ladder compared to day 1 and day 5 of sampling. 38

Table 9. One-way ANOVA table for the inter-day variability of the hydrocarbon ladders on the

Shimadzu GC ................................................................................................................. 39

Table 10. Intra-day variability of retention times of 24 replicates of hydrocarbon ladder using

Method 2 parameters.................................................................................................... 45

Table 11. Inter-day variability of hydrocarbon ladder compared to day 1 and day 5 of sampling

on the PerkinElmer GC ............................................................................................. 47

Table 12. One-way ANOVA table for the inter-day variability of the hydrocarbon ladders on the

PerkinElmer GC ............................................................................................................. 48

Table 13. Intra-day variability of retention times of 3 replicates of the hydrocarbon ladder using

Method 3 parameters.................................................................................................. 52

Table 14. Student t-test at 95\% confidence interval for hydrocarbon ladder comparing Method 2

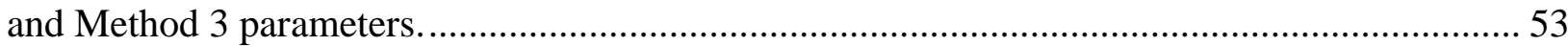

Table 15. Paired t-test at 95\% confidence interval for hydrocarbon ladder comparing Method 2

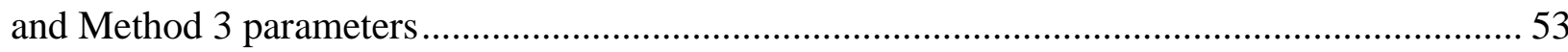

Table 16. Combined linear retention index data on the Shimadzu GC. .................................. 54

Table 17. Uncertainty range for stimulant on the Shimadzu GC.............................................. 58

Table 18. Modified combined data for methamphetamine and phentermine on the Shimadzu GC

Table 19. Modified uncertainty range of LRI for methamphetamine and phentermine on the

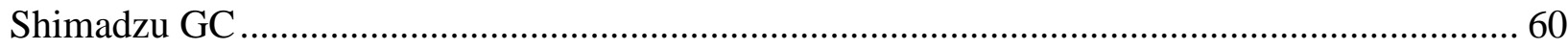

Table 20. Uncertainty range for barbitals on the Shimadzu GC........................................... 61

Table 21. Uncertainty range for depressants on the Shimadzu GC ........................................ 62

Table 22. Combined linear retention index data on the PerkinElmer GC ............................... 63

Table 23.Uncertainty range for stimulants on the PerkinElmer GC ....................................... 64

Table 24. Uncertainty range for barbitals on the PerkinElmer GC........................................... 65

Table 25. Uncertainty range for depressants on the PerkinElmer GC ..................................... 66

Table 26. LRI data for stimulants on the PerkinElmer GC using Method 3 parameters ............. 68

Table 27. LRI data for barbitals on the PerkinElmer GC using Method 3 parameters................ 69

Table 28. LRI data for depressants on the PerkinElmer using Method 3 parameters ................. 70

Table 29. One-way ANOVA table for pentobarbital from both instruments ............................ 71

Table 30. LRI of 10 ppm mixture of methamphetamine and phentermine on the Shimadzu GC 72 


\section{List of Figures}

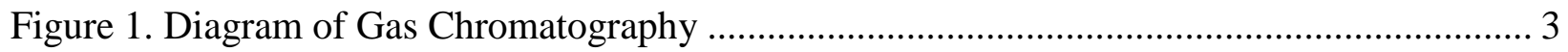

Figure 2. Diagram of ion source and mass analyzer in Mass Spectrometry ………....................... 6

Figure 3. Diagram of hydrocarbon ladder and target analyte ................................................. 9

Figure 4. Hydrocarbon ladder under isothermal conditions ....................................................... 10

Figure 5. Plot of Carbon number plotted against retention time at $150^{\circ} \mathrm{C}$ for the hydrocarbon

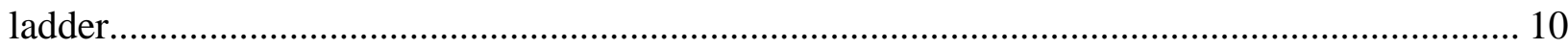

Figure 6. Plot of Carbon number against average retention time of 24 replicates of hydrocarbon

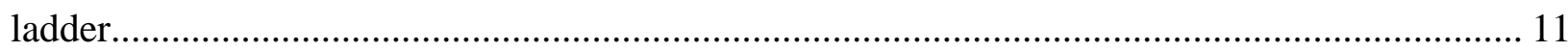

Figure 7. Hydrocarbon ladder C11-C40 on the Shimadzu GC .................................................... 29

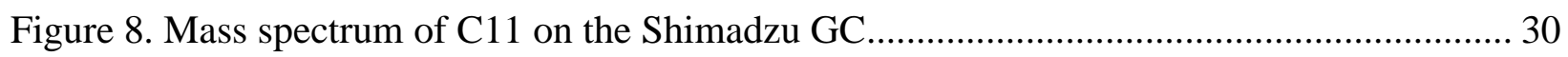

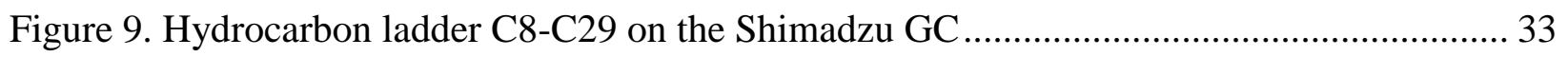

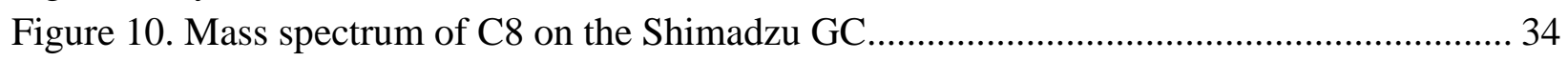

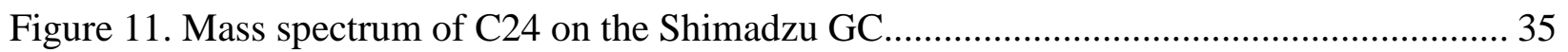

Figure 12. Hydrocarbon ladder C9-C33 on the PerkinElmer GC.............................................. 40

Figure 13. Mass spectrum of C7 on the PerkinElmer GC ……............................................... 41

Figure 14. Mass spectrum of $\mathrm{C} 24$ on the PerkinElmer GC ...................................................... 41

Figure 15. Hydrocarbon ladder C10-C33 on the PerkinElmer GC............................................. 43

Figure 16. Mass spectrum of $\mathrm{C} 10$ on the PerkinElmer GC ..................................................... 44

Figure 17. Mass spectrum of C24 on the PerkinElmer GC.................................................... 44

Figure 18. Hydrocarbon ladder C10-C33 on the PerkinElmer GC............................................. 49

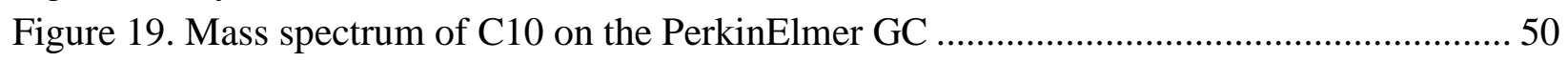

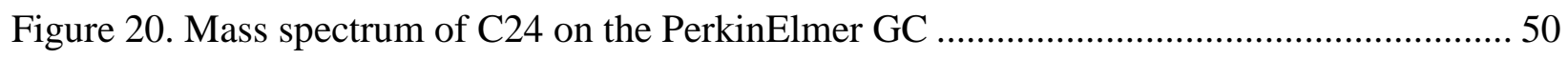

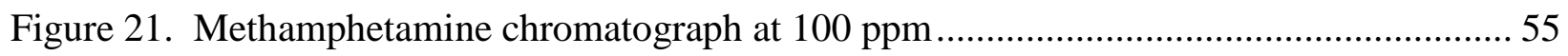

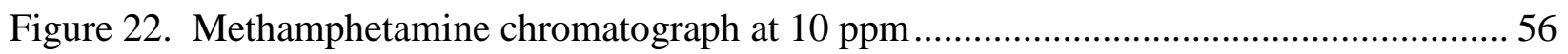

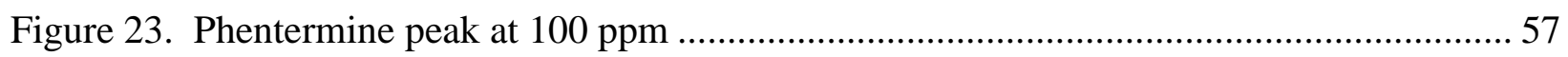

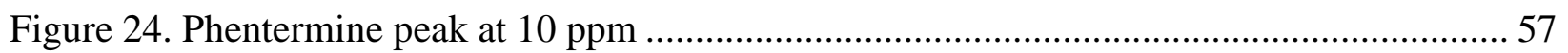

Figure 25. Methamphetamine and phentermine at $10 \mathrm{ppm}$ on the Shimadzu GC ........................ 73

Figure 26. Methamphetamine and phentermine at $100 \mathrm{ppm}$ on the Shimadzu GC ...................... 73

Figure 27. Methamphetamine and phentermine at $10 \mathrm{ppm}$ on the PerkinElmer GC.................... 74

Figure 28. Methamphetamine and phentermine at $100 \mathrm{ppm}$ on the PerkinElmer GC................... 75 


\section{Introduction}

In 2011, over 1.5 million people were arrested for drug abuse (FBI, 2011). The highest arrest rate was for drug abuse violations which involved manufacturing, selling, and possession of controlled substances. Requests for drug analysis accounted for nearly half of all requests (DNA, toxicology, latent prints, etc.) submitted to forensic laboratories in the United States. According the Drug Enforcement Agency (DEA) (2011), marijuana/THC was the most frequently identified controlled substance followed by cocaine, methamphetamine, and heroin. The detection of amphetamines has nearly doubled and oxycodone has quadrupled in the past ten years. According the a study funded by the National Institute of Justice (NIJ) found that laboratories are able to process about $80 \%$ of their controlled substances requests (Storm et al., 2010). However, there is still an estimated backlog of around 220,000 cases per year. This backlog accounts for nearly half of the total backlog of forensic laboratories. If a technique could be used to shorten drug analysis turnaround, then the backlog of cases could be decreased.

The use of gas chromatography/mass spectrometry (GC/MS) in forensic drug analysis can present problems in possible misidentification of new designer drugs or false positive in analytes with nearly identical mass spectra. GC/MS analysis combines two techniques to identify an unknown mixture or sample. The gas chromatograph (GC) serves to separate components in the sample via analyte retention to the column retention of the column prior to introduction into the mass spectrometer. GC analysis alone cannot provide definitive identification of an analyte, but the retention time still provides useful information. The Scientific Working Group for the Analysis of Seized Drugs (SWGDRUG) defines the GC method as a category B analytical technique (SWGDRUG, 2013). This means that the discriminating power of the GC retention time alone is less than that of a category A analytical 
technique. SWGDRUG defines the MS as a category A technique, which means that the discriminating power of the technique is high. In contrast, a category $\mathrm{C}$ technique, such as color tests, has a low discriminatory power because they can only give an idea of what the drug structure may contain as it only tests for the presence of particular functional groups. Therefore, GC retention time could provide useful information even if not a definitive identification.

Mass spectrometry can provide identification through the use of spectral matching from a library or database such as from the National Institute of Standards and Technology (NIST). SWGDRUG also recommended that when a category A analytical technique is used, an analytical technique from the other three categories (A, B, or C) should also be used. The combination of the GC and MS meets this requirement and thus provides identification of a given compound as long as the appropriate reference materials are used.

Gas chromatography combines a carrier gas, typically helium or hydrogen, with a stationary phase or silica-based column (See Figure 1). The most common injector used in forensic application for the gas chromatograph is a split/splitless injector. The components of the sample are vaporized in the gas chromatograph injector port and pushed through the column by the carrier gas. The carrier gas is inert and does not play a role in the partitioning process. The compound volatizes into the carrier gas in the heated injector port and is then forced through the column. The components of a sample are separated based on the relative affinity of the analyte for the stationary phase. The interactions between the analyte and stationary phase cause the components of the sample to elute from the column at different times, called the retention times. The retention times should be reproducible under the same experimental conditions and stationary phase but the specificity depends on the detector. No structural information is revealed by a retention time alone. 


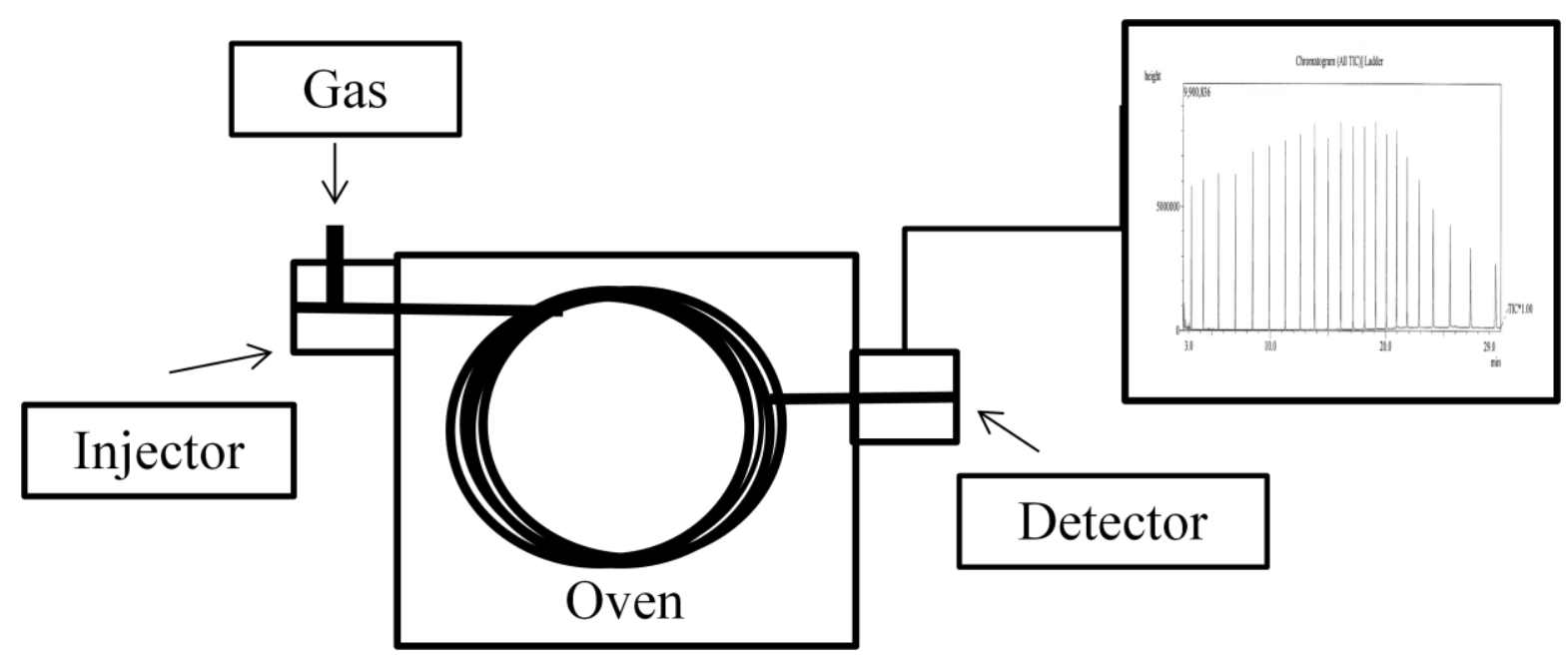

Figure 1. Diagram of Gas Chromatography

The most common columns used in forensic science are DB-1 (100\% dimethylpolysiloxane) and DB-5 (5\% phenyl 95\% methylpolysiloxane) with different vendors using different labeling schemes. DB-1 and DB-5 columns are both considered to be non-polar columns. DB-5 columns are slightly more polar compared to DB-1 columns because of the 5\% phenyl addition to the column. Analytes in the mixture separate in non-polar columns based on their affinity for the phase. DB-1 columns have a low selectivity which means that analytes are separated primarily by their boiling point. This is advantageous to drug analysis, because it will separate a variety of drugs. Amine columns can also used for the analysis of basic drugs. These columns allow for the analysis of basic drugs without having to derivatize them first. These columns are not used as often in forensic laboratories compared to DB-1 and DB-5.

Capillary columns are used in drug analysis. The liquid stationary phase is coated on the wall of the column. The column diameter, film thickness, and column length should be considered when choosing the most efficient column for drug analysis. The diameter of the column affects carrier gas flow rate. Generally, columns with a smaller inner diameter will 
provide a more efficient separation. An increased carrier gas pressure is needed to accommodate smaller diameter columns. The length of the column affects the retention time, column efficiency, and flow resistance. The film thickness effects the interactions with the analyte(s) in the sample. A thicker film provides a higher capacity for the analyte(s) to partition into and thus increases the overall retention that is possible. A thicker film would be used for analytes that are highly volatile while a thinner film can be more suitable for analytes with a high boiling point and molecular weight. The temperature of the column in the oven should also be considered.

In chromatography, the ideal is a narrow symmetrical peak that is Gaussian in shape. The Plate theory is often used to describe column efficiency and the model is derived from the distillation theory. The theoretical plates are measured from the width of the peak in the chromatograph. The more plates a column has, the narrower the peaks are, and therefore the more efficient the column is. The van Deemter equation (Eq. 1.1) describes the factors that contribute to peak broadening and thus influence the theoretical plate height ( $\mathrm{H}$ or HETP, height equivalent of a theoretical plate).

$$
\mathrm{H}=\mathrm{A}+\mathrm{B} / \mathrm{u}+\mathrm{Cu}
$$

The A term is the Eddy diffusion term. This is defined as the multiple paths an analyte can take through the column. This term is negligible in capillary columns and is ignored, but was important in packed columns that dominated early GC instrumentation. Although packed columns are still used in some applications, they are rarely used in forensic analyses. The B term is the longitudinal diffusion term. Analytes have a tendency to diffuse from higher concentrations to low dilute concentrations. The longer the analyte is on the column, the more time it has to diffuse and cause the peak to broaden. At high flow velocities (term u), this term is 
negligible. However, when the flow velocity is raised, the $\mathrm{C}$ term (resistance to mass transfer) is affected. The $\mathrm{C}$ term describes the interactions with between the analyte and the stationary phase. For example, a column with a thicker film thickness will have a higher $\mathrm{C}$ term due to more binding sites being available. Often, these terms are plotted to find the maximum column efficiency which is met when $\mathrm{H}$ (plate height) is small.

After the sample elutes from the GC column, it enters the detector where an electrical signal is created. The means of creating this signal depends on the detector. There are several detectors that can be used in combination with the GC. Two that are commonly used in drug analysis are a mass spectrometer (MS or MSD) and a flame ionization detector (FID). This detector involves the use of a flame supplied from hydrogen and air. The analyte is ionized in the flame and the resulting ion current is measured by a collection electrode to produce a chromatograph. The detector is mass sensitive, and is only responsive to combustible carbon compounds. If a small amount of carbons are present, a small peak will be generated. The technique is highly sensitive and rugged. This detector is limited in that it will not detect most compounds that are not hydrocarbons. Therefore, it does not detect water. A disadvantage to the technique is the sample is destroyed in the process of detection. Due to its robustness, the GC/FID is often used as a screening tool in drug analysis. However, since this project focuses on mass spectrometry, no further discussion of FID will be presented.

The main components of a mass spectrometer are displayed in Figure 2. An electron ionization source is most commonly used in GC. In these designs, a filament produces electrons that are attracted to an electrode held at 70 electron volts $(\mathrm{eV})$ with the entire detector kept under vacuum (on the order of $10^{-5}$ Torr or $10^{-3} \mathrm{~Pa}$ ). As the compounds exit the capillary column, they travel past the electrons produced by the filament. The electrons cause ionization and 
fragmentation. As long as the ionization energy is the same and the MS is performing nominally, the fragmentation pattern should be reproducible. The charged ions that exit the ion source, are focused into a beam via lenses, and accelerated into the mass analyzer. The ionized components are attracted toward the mass analyzer, typically a quadrupole design.

A quadrupole mass analyzer is comprised of four rods that produce an electromagnetic field that allow ions with stable trajectories to pass through. DC currents and radiofrequencies are applied to the rods which allow for selected ions to pass through. The unstable ions are not able to pass the length of the rods and will not reach the detector. The detector converts ions to electrons and amplifies the electron signal. When quadrupole voltages are scanned, the detector response can be plotted as a function of voltage to produce a mass spectrum. Since the fragments are distinctive of the compound, the fragments allow for identification of the analyte. This technique is considered a hard ionization technique because of the extent of fragmentation.

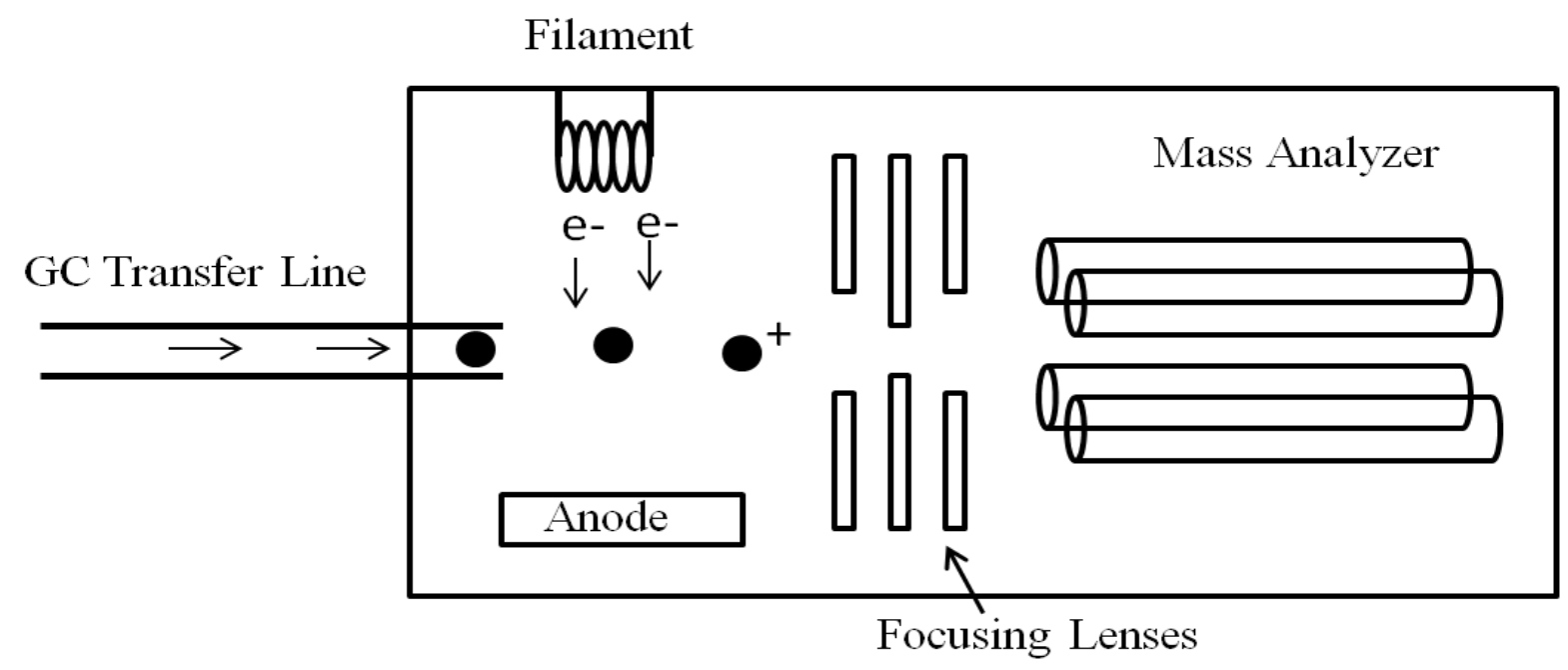

Figure 2. Diagram of ion source and mass analyzer in Mass Spectrometry

In general, the first step toward identification of a compound that elutes from the GC is an evaluation of the mass spectrum searched against an extensive library of references. The 
library will then generate a list of potential matches along with a measure of similarity between the sample spectrum and the library entry. Forensic laboratories typically utilize a mass spectral library published by the National Institute of Standards and Technology (NIST). Difficulties arise when the authentic spectrum is not in the database such as the case with "designer drugs" such as the synthetic cannabinoids, cathionones, and so-called "bath salt" compounds. Designer drugs are often derivatives of illegal drugs that are created to obtain similar effects of the illicit substance without the legal implications. Bath salts are said to have similar effects to the use of amphetamine and cocaine (DEA 2012). However, they have caused hallucinogenic effects, paranoia, a heart attack, and liver failure. Because so many of the compounds in these groups share similar structures, the mass spectra are often similar. This problem, combined with the lack of standards available for designer drugs, significantly decreases the value of the mass spectrum alone for making an identification.

For example, cathinones or "bath salts" are often amphetamine derivatives in which the molecular ion is barely or not present using MS. A study was done on bath salt derivatives and found two common derivatives (cathinone, methcathinone) had very similar retention times using GC/MS (3.69 and 3.86 minutes respectively) (Locos and Reynolds, 2012). The base peak or peak with the greatest intensity, of cathinone is $\mathrm{m} / \mathrm{z} 44$ which is the same base peak of amphetamine. A derivative, methcathinone has a base peak of $\mathrm{m} / \mathrm{z} 58$ which is the same base peak of methamphetamine (NIST Chemistry WebBook, 2013). If these compounds are searched in the NIST database, a false positive identification to amphetamine or methamphetamine could be produced. RI could differentiate these cathinone derivatives which could reduce false positive identifications especially since reference materials may not be available for all the derivatives of cathinone in the NIST database. 
While GC/MS is used in most laboratories in the United States, the technique does have some limitations in drug analysis. One main limitation of only using mass spectra libraries is that the spectra cannot differentiate between all analytes (Babushok et al. 2007). This can lead to a possible false identification or a possible false positive of a drug sample. For example, phentermine and methamphetamine produce a nearly identical spectra. While both are controlled substances, phentermine is a Schedule IV drug while methamphetamine is a Schedule III to II drug. Schedule III to II drugs have a higher dependency while schedule IV drugs have a lower potential for abuse. The difference in penalty can range from only getting a fine for having a schedule IV drug to spending years in prison for having a Schedule III to II drug. Thus, false positives can have enormous consequences. This is one reason why retention indices still play an important role in seized drug analysis. As the overflow of designer drugs continues and more and more compounds with similar or identical spectra enter the forensic system, retention indices could become vital for screening purposes.

Retention index (RI) was developed in 1958 by Kovats (Zellner et al., 2008). Relative retention times are used in place of absolute retention times for the calculation of retention index. A relative retention time describes the relative position of the unknown retention time between two hydrocarbon's retention times. The unknown is described from its location between two hydrocarbons, making it less subject to experimental changes compared to the raw retention time (See Figure 3). 


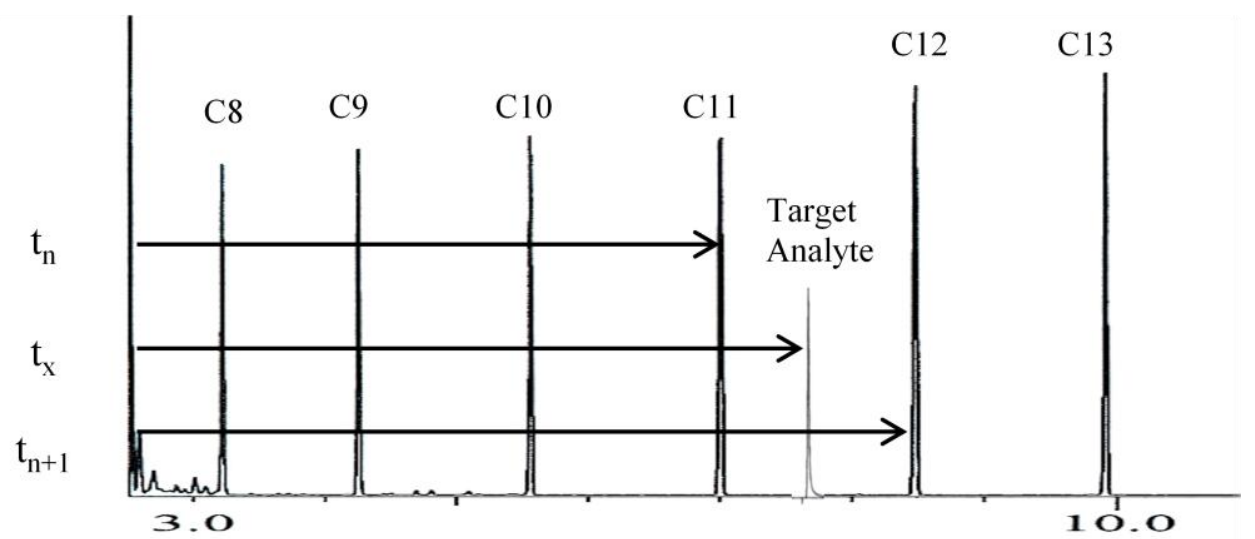

Figure 3. Diagram of hydrocarbon ladder and target analyte

Kovats developed the method to improve peak identification. However, he learned that the larger the span of retention time between the reference and sample peaks, the less accurate the identification was based on retention index alone. Kovats used a homologous series of alkanes now known as a hydrocarbon ladder. Under isothermal conditions, the retention times of the alkanes increased exponentially (See Figure 4). Kovats determined that that a semilogarithmic relationship between the adjusted retention times of the ladder and their carbon numbers existed (See Figure 5). It was defined that the carbon number of the alkane multiplied by 100 was that alkane's retention index. 


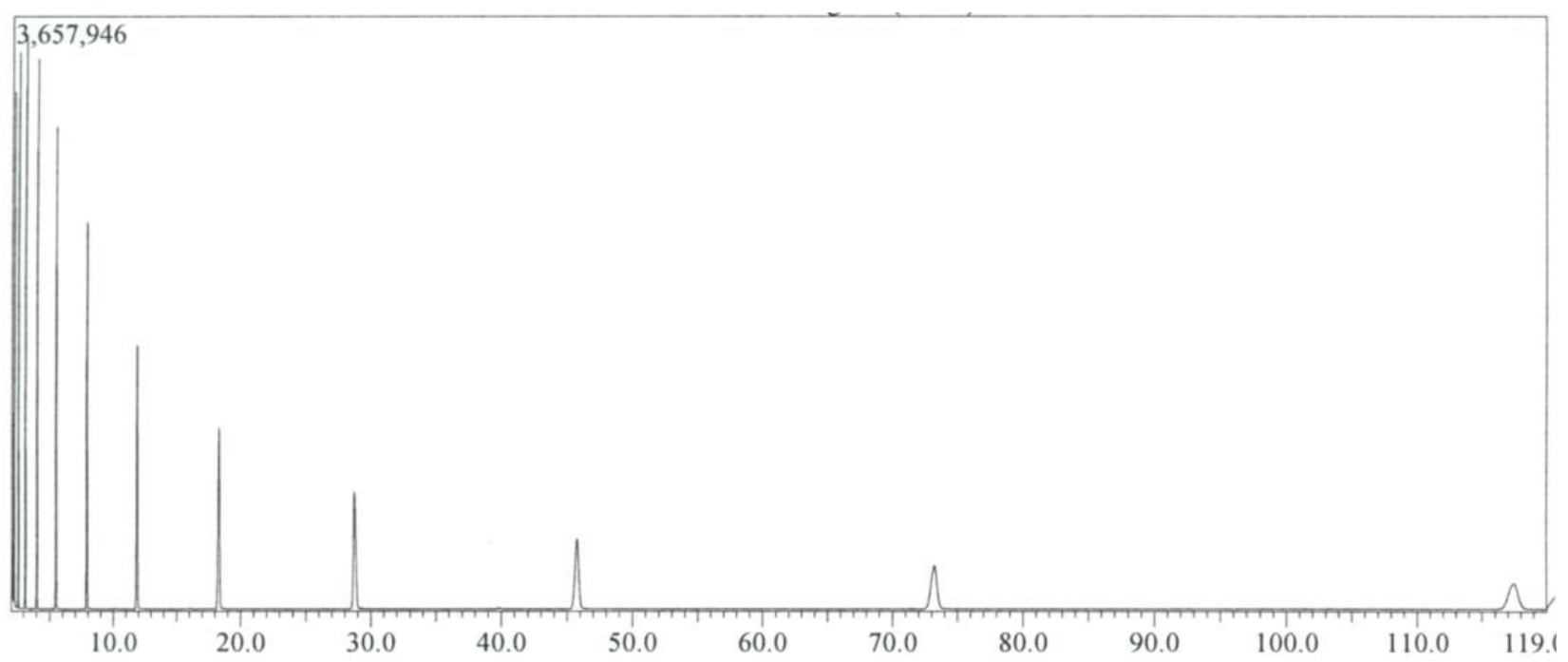

Figure 4. Hydrocarbon ladder under isothermal conditions. A two minute solvent delay was used.

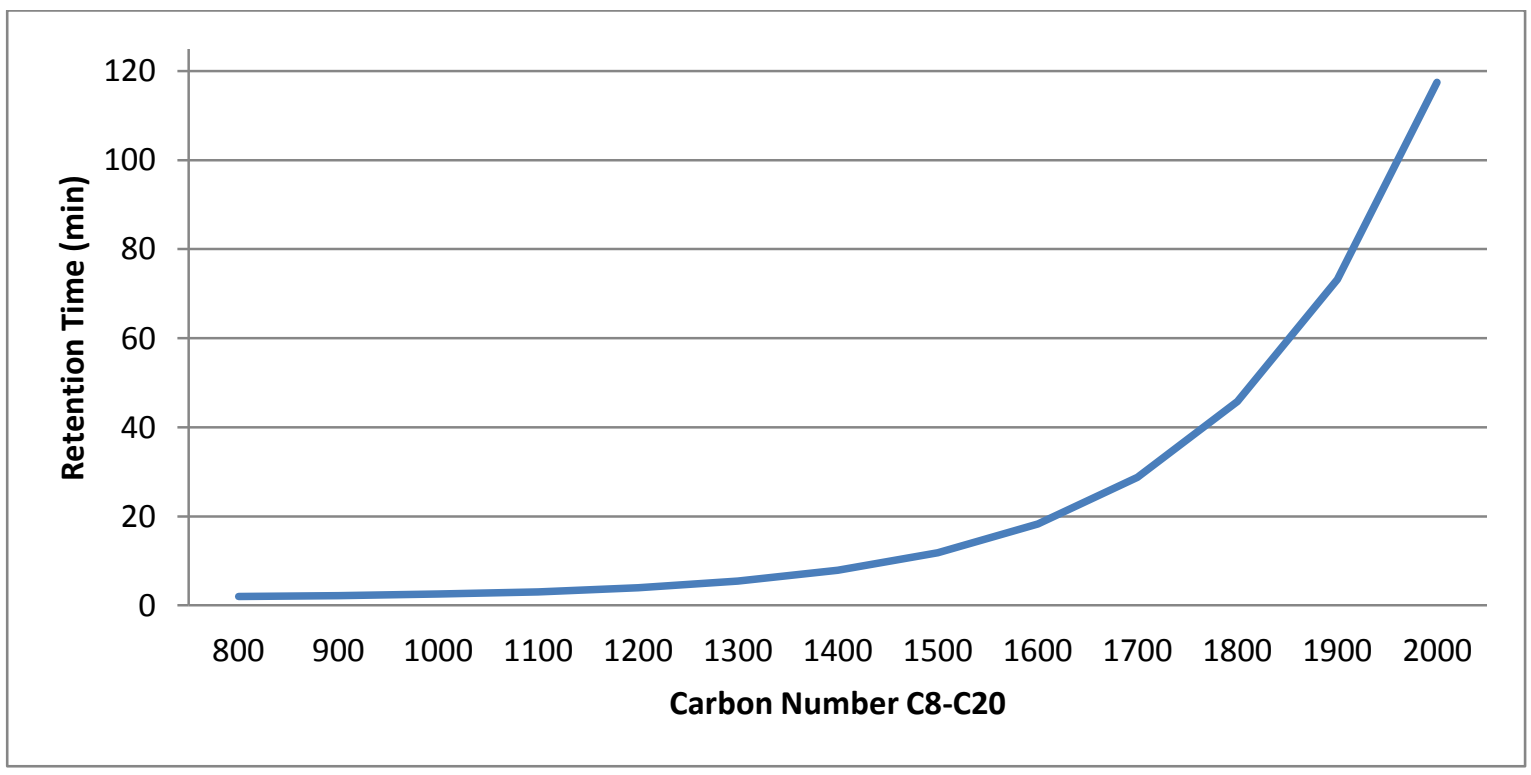

Figure 5. Plot of Carbon number plotted against retention time at $150^{\circ} \mathrm{C}$ for the hydrocarbon ladder

The calculation is shown below for Kovats retention index (I) calculated from isothermal conditions (Eq. 1.2).

$$
I_{x}=100 \mathrm{n}+100\left[\log \left(\mathrm{t}_{\underline{\mathrm{x}}}{ }^{\prime}\right)-\log \left(\mathrm{t}_{\mathrm{n}}{ }^{\prime}\right)\right]
$$


Where $n$ equals the number of $\mathrm{C}$ atoms of the preceding hydrocarbon, $\mathrm{t}_{\mathrm{n}}$ is the preceding hydrocarbon adjusted retention time, $\mathrm{t}_{\mathrm{n}+1}$ is the following hydrocarbon adjusted retention time and $t_{x}$ is the adjusted retention time of the analyte. The advantage to Kovats RI method is that $I_{x}$ is independent of most experimental conditions with the exception of the stationary phase of the column and the isothermal temperature of the column.

With the development and improvement of temperature programming to the GC, a modified version of the equation developed by Kovats was required, known as linear retention indices (LRI). It was shown that when a temperature program was applied, the ladder eluted in a linear mode (See Figure 6).

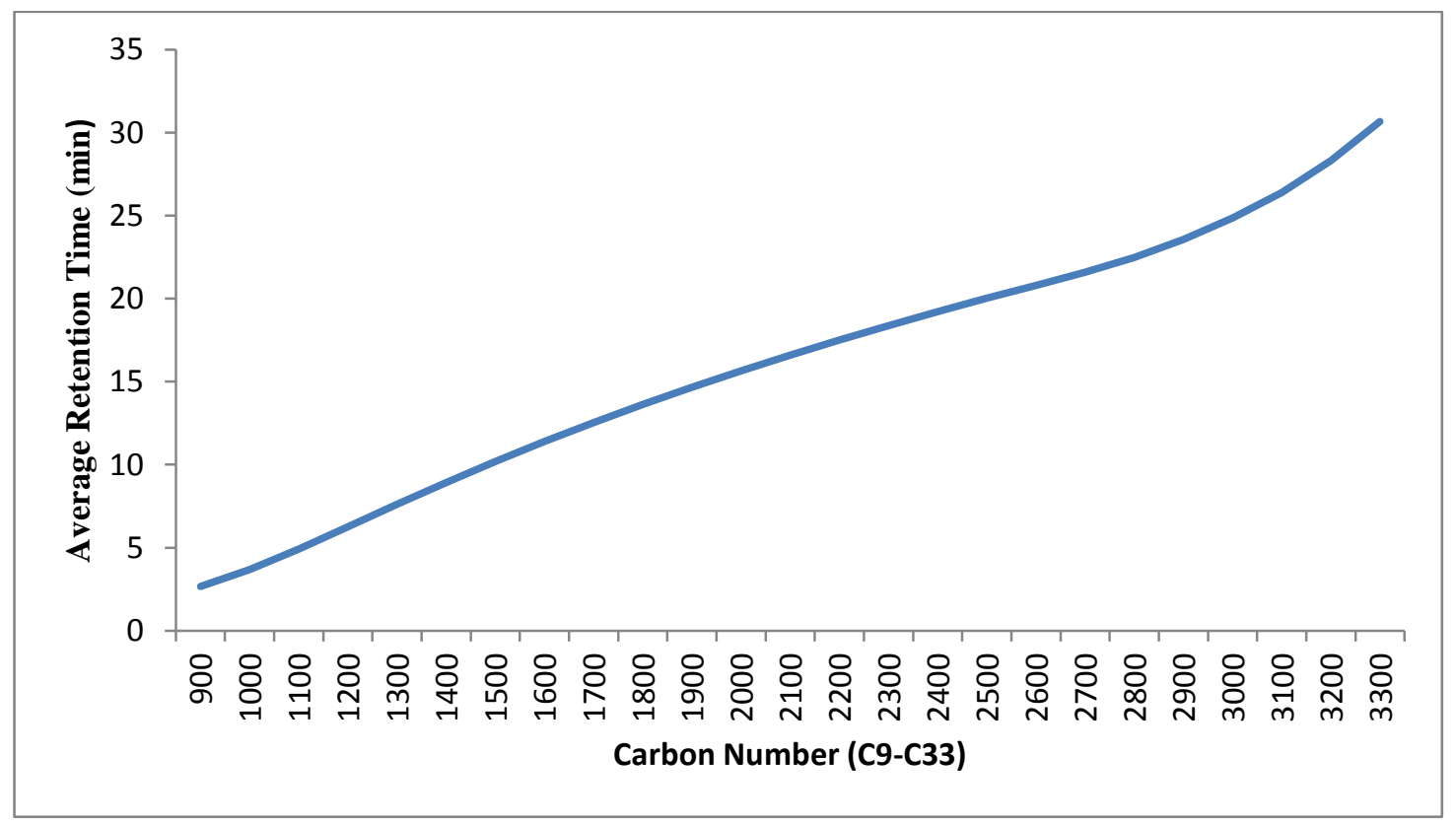

Figure 6. Plot of Carbon number against average retention time of 24 replicates of hydrocarbon ladder. This example was obtained from a Perkin Elmer GC, described in detail the "Methods" section to follow. 
Since the retention times did not increase exponentially, the logarithmic scale could not be used in this equation. The modification was developed by Van den Dool and Kratz (Babushok et al. 2007) and shown below (Eq. 1.3).

$$
\begin{aligned}
& I_{x}=100 \mathrm{n}+100\left(\underline{\mathrm{t}_{\mathrm{x}}} \underline{-\mathrm{t}_{\mathrm{n}}}\right) \\
& \left(t_{n+1}-t_{n}\right)
\end{aligned}
$$

where $t_{n}$ is the preceding hydrocarbon retention time, $t_{n+1}$ is the following hydrocarbon retention time and $t_{x}$ is the retention time of the analyte.

The most commonly used indices are Kovats and Linear RI. Other retention indices exist such as Lee indices, which use polyaromatic hydrocarbon standards. The Lee indices also have different calculations for isothermal conditions and temperature programming conditions. Since most unknown drug samples require the use of temperature programming and it is now more common, linear retention indices were the focus of this work. Linear retention indices are dependent on the stationary phase of the column and the temperature program but when used under the same experimental conditions are reproducible

Babushok et al. (2007) provided an in depth discussion of the development of a database of retention indices from NIST. The authors examined how the database was constructed as well as the type of information that is provided. The database was constructed mostly through peer reviewed publications from 1956-2005. The indices that are reported in the database are Kovats, Linear, and Lee. Most of the data present in the database is calculated from Linear indices followed by Kovats indices. Only a small portion of the data is calculated using the isothermal or non-isothermal Lee indices. The columns described in the database are from non-polar or polar columns. A large portion of the data on non-polar columns was performed on a $100 \%$ dimethylpolysiloxane stationary phase which is seen in DB-1 columns, HP-1, and SE-30 
columns. The second largest portion of data on non-polar columns were performed on methyl silicone, 5\% phenyl groups stationary phase which are seen on DB-5 and HP-5 columns.

With the large time span of the data gathered, quality control measures were developed to try to eliminate erroneous and suspicious data. Some of the problems with assigning the quality control measures were the lack of replicates. About $53 \%$ of the compounds only had one value reported so the uncertainty associated with that value is unknown. A small number (2.4\%) of compounds had 48 or more replicates. The values were only compared if the same experimental conditions were present. The data with sufficient replicates were compared to an estimated value of RI. A value was ruled suspicious if the experimental procedure was not clearly defined, if the source stated problems during the experimental procedure, or if the results of RI contained a large variability with other sources for the same compound. The estimation of RI involved a group additivity scheme used from Stein et al. (2007). If a large variability was present, the conditions in which RI was calculated were examined further and eventually characterized into the quality control definitions of acceptable data, suspicious data, or data very likely in error. Some possible reasons for the large variations for some of the values that were reported could be explained by improperly reporting RI, applying the wrong calculation of RI which could lead to a misidentification of the compound that has been assigned the RI value, and possible different temperature programs used. Only values deemed acceptable are seen in the NIST database for RI. The database is available from NIST Chemistry WebBook and was released in June 2005.

Another study by Toth and Praszna (1998) examined reproducibility of RI on DB-1 columns. The RI studied was calculated using Linear RI. The authors found better repeatability when they applied a correction factor on the hydrocarbon ladder to normalize the ladder. Although, for the correction factor to be applied, the unknown sample has to contain one alkane 
from the hydrocarbon ladder. The unknown sample and the ladder have to have been injected separately as well.

A study by Lebron-Aquilar et al.(2002) showed that Kovats index can generate a repeatability of RI to one-tenth of a retention index unit (riu). This calculation involved a nonlinear equation and can only be used under isothermal conditions. Specific experimental conditions were required to achieve accuracy to one-tenth of a riu. At least five runs of the sample, retention times measured at 0.1 second or $0.001 \mathrm{~min}$, and the non-linear regression formula cited in the paper had to be applied. Given that the consensus values for RI are cited to whole numbers, the ability to report to one decimal place is a significant improvement; the tradeoff lies in the experimental constraints.

Zhang et al. (2011) studied the experimental effects of RI to use a software program called iMatch that uses spectral matching while also using separation information. The authors used information presented in the NIST 2008 RI database to look at several experimental parameters that could influence RI and grouped them based on their impact. The authors were able to group some of the experimental parameters together to ultimately create distribution functions from the grouped RI data. The authors applied the distribution functions into a software program called iMatch to try and eliminate false positives in spectral matching. The most significant findings of their study were that column class, type, and data type do have an impact on RI.

Research currently on RI has been in developing an accurate method for the prediction or estimation of RI values. There is also much interest to having a tool that could increase repeatability for RI. This would allow for a larger amount of analytes to have an estimated retention index. This could ultimately lead to a database with more analytes which could lead to 
identifying unknown samples such as drugs. Currently these values are reported in the database, and often it is seen that the estimated values vary from the experimental values of RI. More research needs to be done in this area before assigning retention indices to all compounds without using experimental values.

The Scientific Working Group for Seized Drugs and Analysis (SWGDRUG) proposed the use of RI to aid in identification on newer designer drugs for which reference materials are not yet available in July 2011 (Placke, July 2011). The goal was to add RI information to the current SWGDRUG MS library and drug monographs (http://www.swgdrug.org/monographs, last accessed April 2013). Data was complied on thirty drug samples to measure the variability of the data between laboratories (Mead, January 2012). Under the same GC and MS conditions, using a HP-1 column, each RI data value was within $2 \%$ of the same data value at each of the three laboratories showing a good inter-laboratory reproducibility (See Table 1). As of this writing, these RI values were being added to the SWGDRUG MS library as of 2013.

Table 1. RI values of 9 illicit drugs over four stationary phases from SWGDRUG.

\begin{tabular}{|c|c|c|c|c|}
\hline Standard Name & $\begin{array}{c}\text { DB-1 12 x 0.20 } \\
\mathbf{x} \mathbf{0 . 3 3}\end{array}$ & $\begin{array}{c}\text { ZB-50 15 x } \\
\mathbf{0 . 2 5} \mathbf{x} \mathbf{0 . 2 5}\end{array}$ & $\begin{array}{c}\text { ZB-5 15 x } \\
\mathbf{0 . 2 5} \mathbf{x} \mathbf{0 . 2 5}\end{array}$ & $\begin{array}{c}\text { DB-1 25 x } \\
\mathbf{0 . 2 0} \mathbf{0 . 3 3}\end{array}$ \\
\hline Methamphetamine & 1174 & 1372 & 1201 & 1183 \\
\hline Amphetamine & 1116 & 1335 & 1145 & 1121 \\
\hline Phentermine & 1156 & 1357 & 1179 & 1164 \\
\hline Pentobarbital & 1729 & 2121 & 1772 & 1730 \\
\hline Butabarbital & 1660 & 2055 & 1694 & 1655 \\
\hline Phenobarbital & 1951 & 2540 & 2016 & 1960 \\
\hline Heroin & 2658 & 3410 & 2744 & 2668 \\
\hline Oxycodone & 2562 & 3330 & 2649 & 2582 \\
\hline Codeine & 2418 & 3095 & 2492 & 2442 \\
\hline
\end{tabular}


According to a recent report on forensic science, the Proceedings of the National Academy of Sciences (NAS) (came out in February '09, copyright July 2009), any laboratory results should be reported with a level of confidence or uncertainty. Some laboratories meet this requirement, but many do not have procedures in place to do this. Presently, RI values are reported as whole numbers, with no associated ranges or uncertainty values. To meet this spirit of the NAS report, uncertainty values should be investigated for RI to increase the level of confidence in reporting RI.

Estimated values of RI are shown in the NIST MS Search 2.0 database (www.swgdrug.org) as well as published RI values. As an example, consider methamphetamine which has an estimated value of 1226 index units using this database. Two published sources list lower values of 1175 iu (Christ et al., 1988) and 1150 iu (Oyama et al., 1987). If a forensic laboratory determines an RI value of 1160 for a peak thought to be methamphetamine, this data would likely not be comparable to the NIST database, nor to the published values unless uncertainties are provided for all four measured RIs. Also, the estimated RI value is much higher than the two experimental values, a discrepancy that was discussed earlier. Clearly, for LRI to be of value across forensic laboratories, uncertainty ranges will be essential, particularly for structurally similar designer drugs that are expected to have similar mass spectra as well as similar retention times. In these cases, small differences in retention time could be important. This project was geared toward providing uncertainty estimations and a statistical investigation of underlying factors that contribute to this variation. 


\section{Drug Standards}

Nine drug standards were examined in this study, falling under the groups of stimulants (See Table 2), depressants (See Table 3), and narcotics (See Table 4). An attempt was made to include acidic and basic drugs with a range of $\mathrm{pK}_{\mathrm{a}}$ values.

Methamphetamine is also known as desoxyn, d-desoxyephedrine, crystal meth, ICE, crank, or speed (DEA 2012). In large doses, methamphetamine may have a hallucinogenic effect. Prescription names for amphetamine are Adderall, Obetrol, and Dexedrine. This is commonly used in the treatment of attention deficit hyperactivity disorder (ADHD). Phentermine has less of a stimulant effect compared to the other two drugs but is an isomer of methamphetamine. 
Table 2. Drug table of stimulants

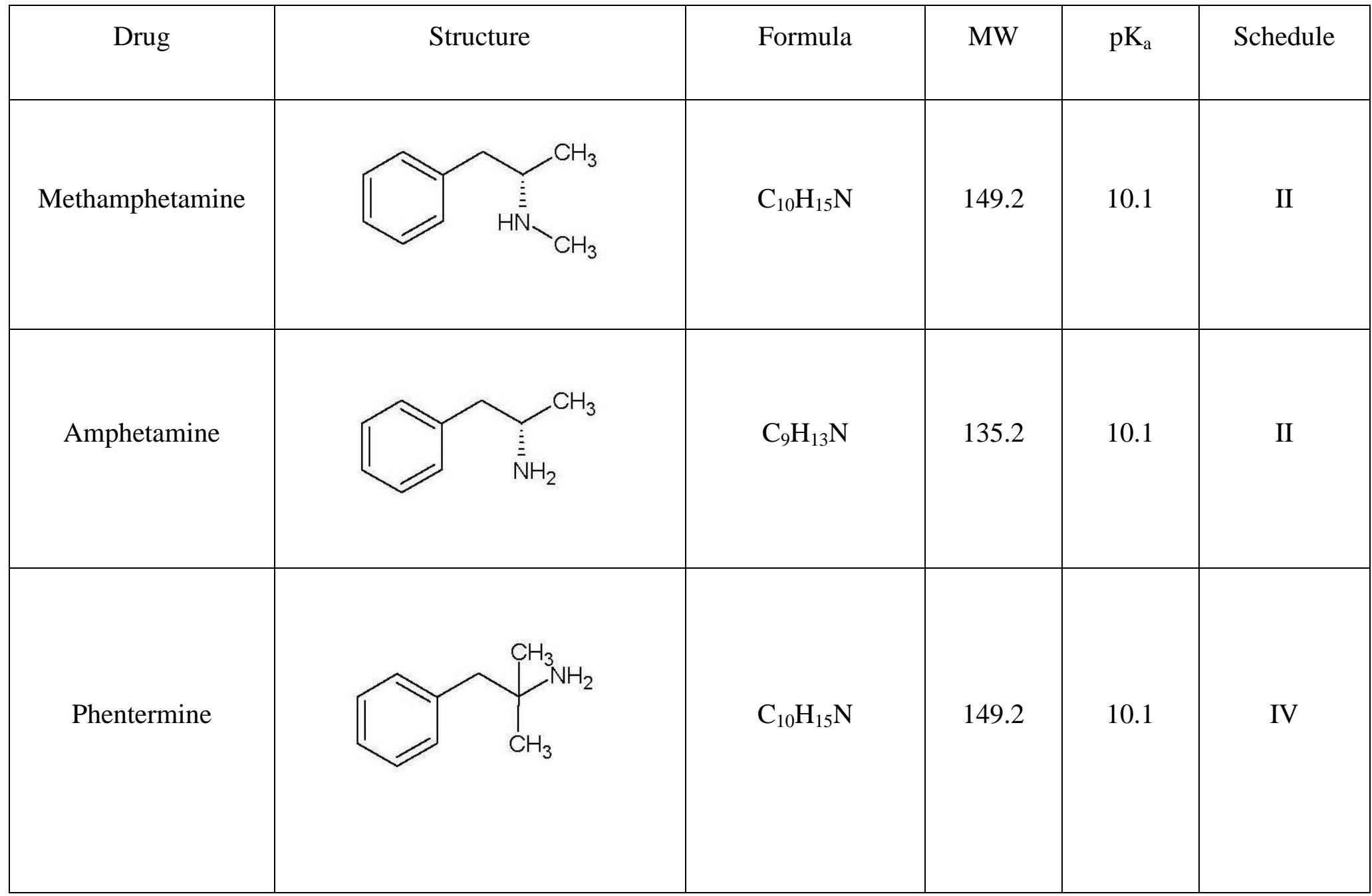

Referenced from: Clarke's Analysis of Drugs and Poisons, 2004 
Barbiturates are drugs that are used to depress the central nervous system and have fallen out of popularity. They are used for insomnia, to relieve muscle spasms, and to calm. These drugs develop tolerance quickly and are classified as ultrashort, short, immediate, and longacting (Levin 2010).

Pentobarbital is a short acting drug compared to the other two drugs and also goes by the trade name Nembutal. Butabarbital is commonly used to treat migraines and insomnia since it is short acting. Some common names are Sonabarb, and Soneryl (DEA 2012). Phenobarbital is a longer acting drug and commonly used to treat seizures. Some prescription names for this drug are Aparoxal, Comizial, and Solfoton. Although these drugs are not subject to abuse to the extent they were twenty years ago, they were included in this study to represent acidic drugs 
Table 3. Drug table of barbitals

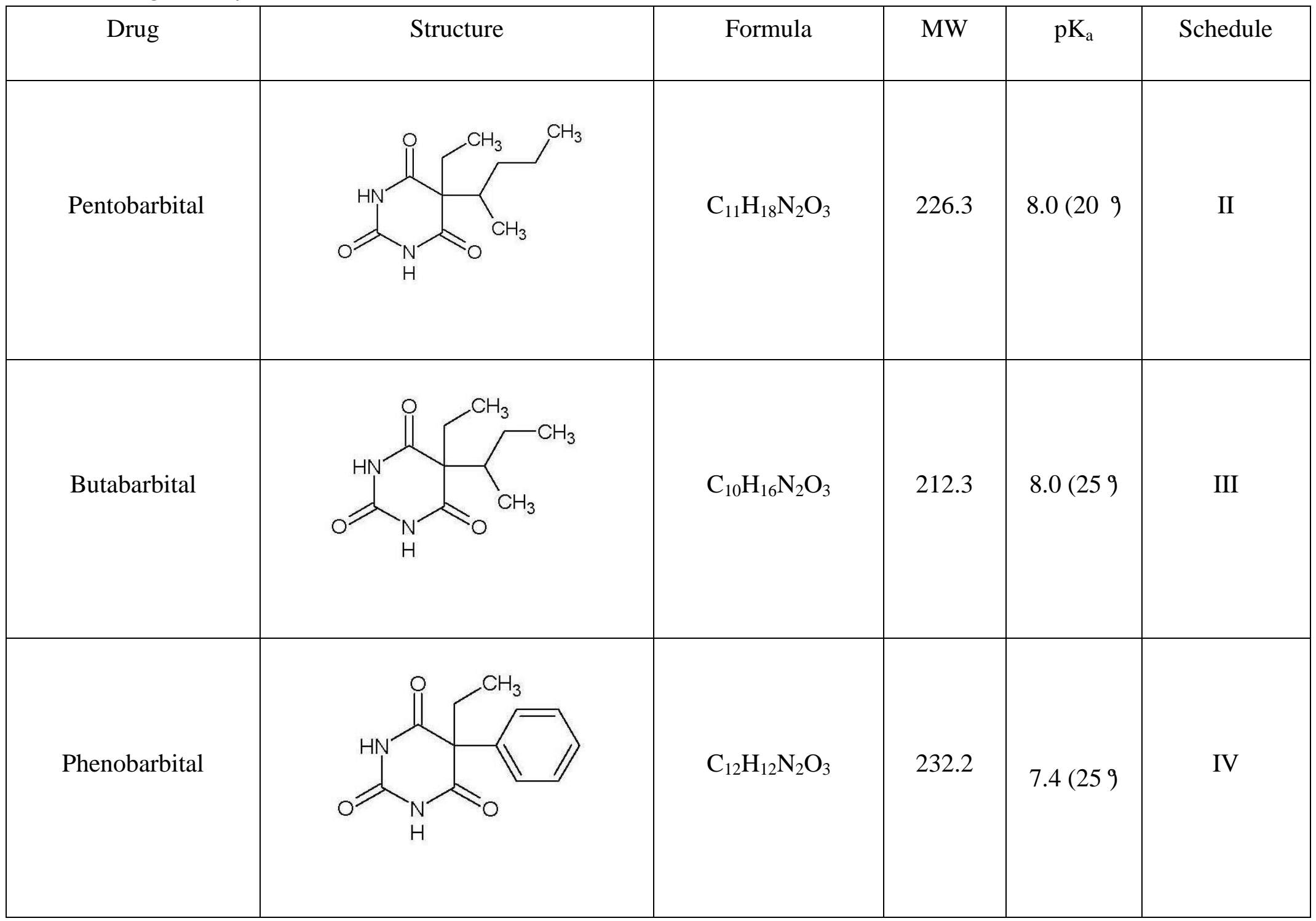

Referenced from: Clarke's Analysis of Drugs and Poisons, 2004 
The narcotics (opiate) contained three separate drugs standards of heroin, oxycodone, and codeine. Opiates affect the central nervous system, dull senses, and relieve pain. Some common uses for opiates include analgesia, trauma, and pain management.

Heroin is also known as diacetylmorphine and diamorphine (DEA 2012). Oxycodone is typically prescribed for pain management. Some prescription names are OxyContin, Percocet, Endocet, Roxicodone, and Roxicet. Codeine can typically be prescribed for injuries and musculoskeletal issues. Some common names include morphine methyl ester and methyl morphine. 
Table 4. Drug table of depressants

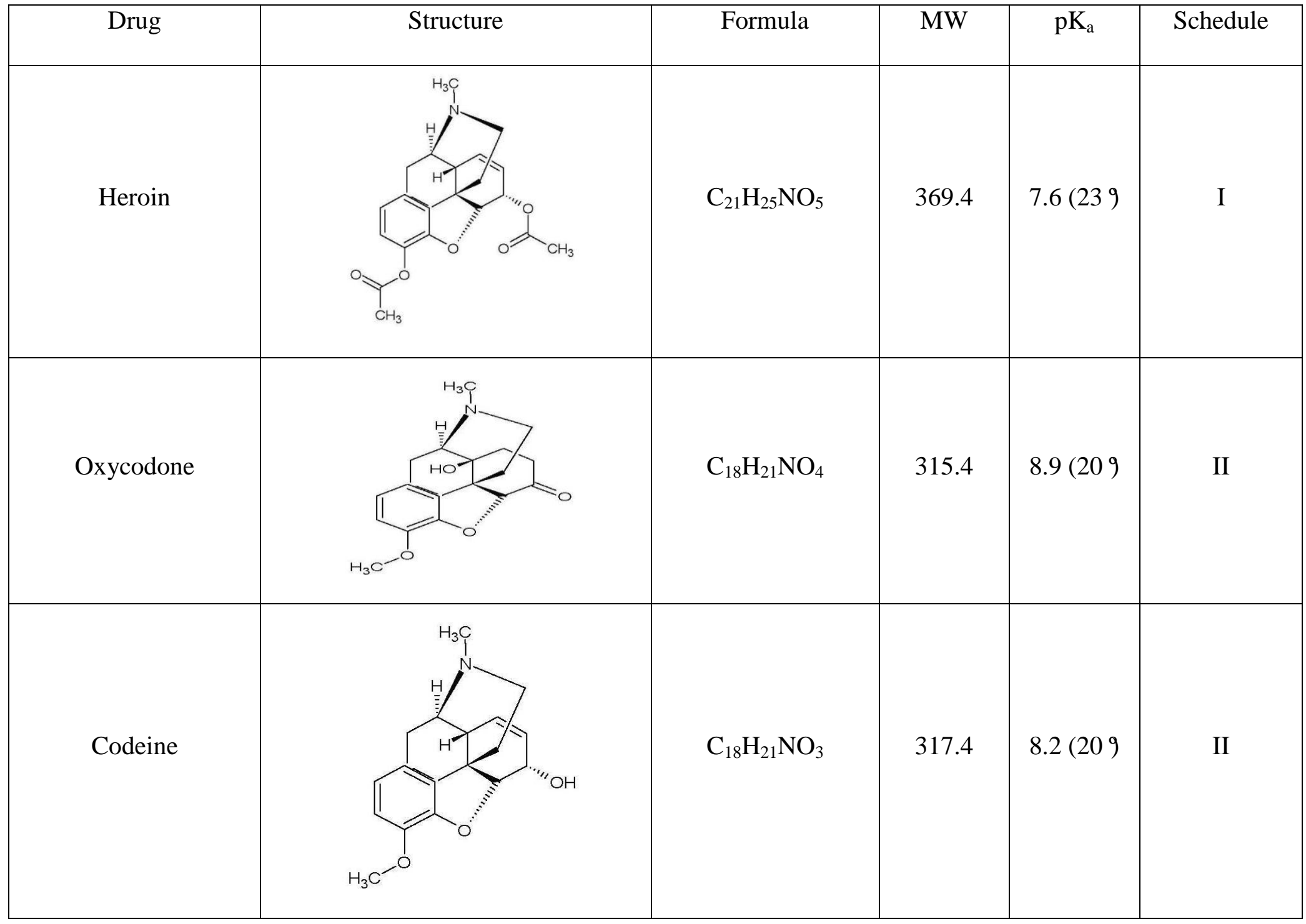

Referenced from: Clarke's Analysis of Drugs and Poisons, 2004 


\section{Objectives}

The first objective was to determine the repeatability (same instrument, same conditions) and reproducibility (between two gas chromatographs of different manufacturer under same temperature programming) of the nine drug standards. Nine drug standards will be used to test variability of linear retention indices between two columns of same stationary phase made by different manufacturers and test the reproducibility of RI between the two instruments. The column used was a 5\% phenyl $95 \%$ methylpolysiloxane stationary phase.

The second objective was to estimate the uncertainty of the LRI of the nine drug standards. A traditional hydrocarbon ladder was used for the columns to generate uncertainty values for each drug so that the variability of LRI between columns could be examined.

Linear retention indices were applied using the retention time of the apex (top) of the peaks from the proceeding and following hydrocarbons. The same stationary phase, temperature program and carrier gas were used on two different gas chromatograph mass spectrometers. Analyses were performed on a Shimadzu and PerkinElmer GCs.

\section{Significance to Forensic Science}

Retention indices are used is forensic laboratories today for screening of compounds and as an additional aid for the identification of compounds. LRI can differentiate between two analytes (such as LSD and LAMPA or methamphetamine and phentermine), that have give similar spectra. This could be useful when analyzing designer drugs, specifically synthetic cannabinoids and bath salts. 
The inter-laboratory values of LRI need to be examined to assess the variability and reproducibility of LRI. Many limitations or concerns that need to be addressed deal with the standardization of the experimental parameters that influence the retention times used to calculate LRI. Since many laboratories use LRI for the screening of drugs, it is essential to define quantitatively repeatability and reproducibility values to determine if inter-laboratory use is even possible. If a reliable database can be developed, with a known confidence interval, the reliability in the approach of LRI would become another tool in the forensic community for presumptive and confirmatory screening. 


\section{Methods}

\section{Instrumentation}

Sampling was performed on two GC/MS instruments.

Table 5. Parameters of instruments

\begin{tabular}{|c|c|c|c|}
\hline & Method 1 & Method 2 & Method 3 \\
\hline Instrument & $\begin{array}{c}\text { Shimadzu GC-2010 } \\
\text { with a QP2010S Mass } \\
\text { Spectrometer }\end{array}$ & $\begin{array}{c}\text { PerkinElmer Clarus } \\
500 \text { GC with a Clarus } \\
\text { SQ8T Mass } \\
\text { Spectrometer }\end{array}$ & $\begin{array}{c}\text { PerkinElmer Clarus } \\
500 \text { GC with a Clarus } \\
\text { SQ8T Mass } \\
\text { Spectrometer }\end{array}$ \\
\hline Column Type & $\begin{array}{l}\text { Rxi-5SiMS w/ Integra- } \\
\text { Guard }\end{array}$ & ZB-5 MSi & ZB-5 MSi \\
\hline Column Length & $\begin{array}{c}30 \mathrm{~m} \times 0.25 \mathrm{~mm} \times 0.50 \\
\text { um }\end{array}$ & $\begin{array}{c}30 \mathrm{~m} \times 0.25 \mathrm{~mm} \times \\
0.25 \mathrm{um}\end{array}$ & $\begin{array}{c}30 \mathrm{~m} \times 0.25 \mathrm{~mm} \mathrm{x} \\
0.25 \mathrm{um}\end{array}$ \\
\hline Manufacturer of Column & $\begin{array}{c}\text { Restek }^{\circledR} \\
\text { (Bellefonte, PA) }\end{array}$ & $\begin{array}{l}\text { Phenomenex }{ }^{\circledR} \\
\text { (Torrance, } \mathrm{CA} \text { ) }\end{array}$ & Phenomenex $^{\circledR}$ \\
\hline Temperature Program & $\begin{array}{c}80^{\circ} \mathrm{C}(1 \mathrm{~min}) @ 10 \\
{ }^{\circ} \mathrm{C} / \mathrm{min} \text { to } 280^{\circ} \mathrm{C}(10 \\
\text { minutes })\end{array}$ & $\begin{array}{c}80^{\circ} \mathrm{C}(1 \mathrm{~min}) @ 10 \\
{ }^{\circ} \mathrm{C} / \mathrm{min} \text { to } 280^{\circ} \mathrm{C}(10 \\
\text { minutes })\end{array}$ & $\begin{array}{c}80^{\circ} \mathrm{C}(1 \mathrm{~min}) @ 10 \\
{ }^{\circ} \mathrm{C} / \mathrm{min} \text { to } 280^{\circ} \mathrm{C}(10 \\
\text { minutes })\end{array}$ \\
\hline Split Ratio & 10: 1 & 10: 1 & 10: 1 \\
\hline Injection Temperature & $280^{\circ} \mathrm{C}$ & $250^{\circ} \mathrm{C}$ & $280^{\circ} \mathrm{C}$ \\
\hline Ion Source Temperature & $260^{\circ} \mathrm{C}$ & $260^{\circ} \mathrm{C}$ & $260^{\circ} \mathrm{C}$ \\
\hline Interface Temperature & $270^{\circ} \mathrm{C}$ & $280^{\circ} \mathrm{C}$ & $270^{\circ} \mathrm{C}$ \\
\hline Column Flow & $1.00 \mathrm{~mL} / \mathrm{min}$ & $1.00 \mathrm{~mL} / \mathrm{min}$ & $1.00 \mathrm{~mL} / \mathrm{min}$ \\
\hline Carrier Gas & Helium & Helium & Helium \\
\hline Voltage of Source & $70 \mathrm{eV}$ & $70 \mathrm{eV}$ & $70 \mathrm{eV}$ \\
\hline
\end{tabular}




\section{Materials}

The hydrocarbon ladder used was from Supelco ${ }^{\circledR}$ (Bellefonte, PA). The standard alkane kit included C7 to C40 saturated alkanes in a concentration of $1000 \mathrm{ppm}$. The ladder was diluted to a concentration of $100 \mathrm{ppm}$ using hexane. The drugs used were methamphetamine, amphetamine, phentermine, heroin, oxycodone, codeine, pentobarbital, butabarbital, and phenobarbital. Each drug standard was diluted with methanol. Pentobarbital and butabarbital standards were purchased from Cerillant ${ }^{\circledR}$ (Round Rock, TX). Phenobarbital, D-amphetamine, phentermine, codeine, and Oxycodone hydrochloride were from Sigma Aldrich ${ }^{\circledR}$ (St.Louis, MO) in a powder form. Methamphetamine was from Restek ${ }^{\circledR}$ (Bellefonte, PA).

\section{Experimental conditions}

The test conditions from Supleco ${ }^{\circledR}$ were used first to make sure all the alkanes in the mix were detectable. The column used was Rxi-5SiMS w/ Integra-Guard by Restek ${ }^{\circledR}$. Hexane was used to dilute the ladder from $1000 \mathrm{ppm}$ to $100 \mathrm{ppm}$. The temperature programming used was 35 ${ }^{\circ} \mathrm{C}(2 \mathrm{~min})$ to $250^{\circ} \mathrm{C} @ 10^{\circ} \mathrm{C} / \mathrm{min}$ to $320^{\circ} \mathrm{C}(20 \mathrm{~min}) @ 20^{\circ} \mathrm{C} / \mathrm{min}$. with a split ratio of $25: 1$. The ladder was diluted to concentrations of $10 \mathrm{ppm}$ and $100 \mathrm{ppm}$ to see which concentration was optimal across the ladders. The solvent delay was set at 10 minutes and C11-C40 compounds were detected at a concentration of $100 \mathrm{ppm}$ which gave the most symmetrical and Gaussian shaped peaks. The run was repeated 5 times. The chromatographs and mass spectra from the Shimadzu GC are shown in black, while the chromatographs and mass spectra from the PerkinElmer GC are shown in red. This is how they are displayed in the software of each GC.

A second method was developed to have a shorter total run time of 30 minutes. This method was used to develop a shorter analysis time and lower temperature of the oven. The 
column used was Rxi-5Si MS w/ Integra-Guard. This method was $80^{\circ} \mathrm{C}(1 \mathrm{~min}) @ 10^{\circ} \mathrm{C} / \mathrm{min}$ to $280{ }^{\circ} \mathrm{C}$ (10 minutes), with a split of 10:1. Using this method, peaks were observed for C9-C33 with a solvent delay time of 2.51 minutes. This range included the elution times of all the drugs studied. A solvent delay of 3 minutes was used for the duration of the study due to seeing solvent at a delay of 2.51 minutes. The equilibration time for the Shimadzu GC was 1 minute while the PerkinElmer was 2 minutes. Once the oven temperature reaches the starting oven temperature, it holds the temperature for the equilibration time to reach thermal equilibrium. Results in this study did not suggest that this difference was a factor.

The mass spectrum was used to identify the alkanes. Molecular ions were used until C24. After C24, the molecular ion fell outside of the scan range of 30-350 amu. Alkanes C7 and C8 were not observed and likely eluted during the solvent delay. This did not present any problems for calculating RI for the drugs studied here. To determine intra-day variability and inter-day variability, data was collected on five separate days. Each standard was analyzed in triplicates. The ladder was run three times prior to the drug standards each of the five days. The nine drug standards all were confirmed using the NIST database for mass spectrometry.

\section{Calculation of LRI}

LRI was calculated from the equation below. This is the same equation as formula (Eq. 1.3). A macro was created for SWGDRUG from the Oklahoma State Police using this equation. This was used to calculate the LRI of the drug screen. 


$$
I_{x}=100 \mathrm{n}+100 \underset{\left(\mathrm{t}_{\mathrm{n}+1}-\mathrm{t}_{\mathrm{n}}\right)}{\left.\underline{\mathrm{t}_{\mathrm{n}}}\right)}
$$

$$
\begin{aligned}
& t_{n}=\text { The retention time of the unknown } \\
& t_{x}=\text { The retention time of the preceding hydrocarbon } \\
& t_{n+1}=\text { The retention time of the following hydrocarbon } \\
& n=\text { The number of carbons of preceding hydrocarbon }
\end{aligned}
$$

For example, a methamphetamine sample eluted at 8.50 minutes. The compound falls between $\mathrm{C} 11$ and $\mathrm{C} 12$ with a retention time of 7.03 and 8.50 min respectively. The LRI of methamphetamine would be:

$$
\begin{gathered}
\mathrm{LRI}=[(8.40-7.03) /(8.50-7.03) * 100]+\left[11^{*} 100\right] \\
\mathrm{LRI}=(1.37 / 1.47) * 100+982 \\
\mathrm{LRI}=93.20+1100 \\
\mathrm{LRI}=1193
\end{gathered}
$$

The LRI value is reported to a whole number by convention.

Currently, the determination of uncertainty values for quantitative forensic data is a significant concern, as noted earlier. While it is recognized that detailed approaches such as uncertainty budgets can and are used in the context of seized drug analysis, this approach was not taken here. Rather, the uncertainty (i.e., the range in which a true value is expected to be found with a given confidence) is estimated using a 95\% confidence interval. For initial study and comparison purposes, this approach was deemed reasonable. 


\section{Results and Discussion}

\section{Hydrocarbon ladder-Shimadzu GC}

The temperature program provided by Supleco ${ }^{\circledR}$ was used to ensure all alkanes were present. Alkanes C11-C40 were present on the Shimadzu GC using a solvent delay of 10 minutes (See Figure 7). This ladder was run in five replicates to measure repeatability. This temperature program was only used to ensure the alkane ladder was complete. The \%RSD for all peaks were less than 0.01 or $1 \%$. The temperature program was not suitable for the rest of the data collection to do the high temperature of $320^{\circ} \mathrm{C}$ for 20 minutes and a total run time of 47 minutes.

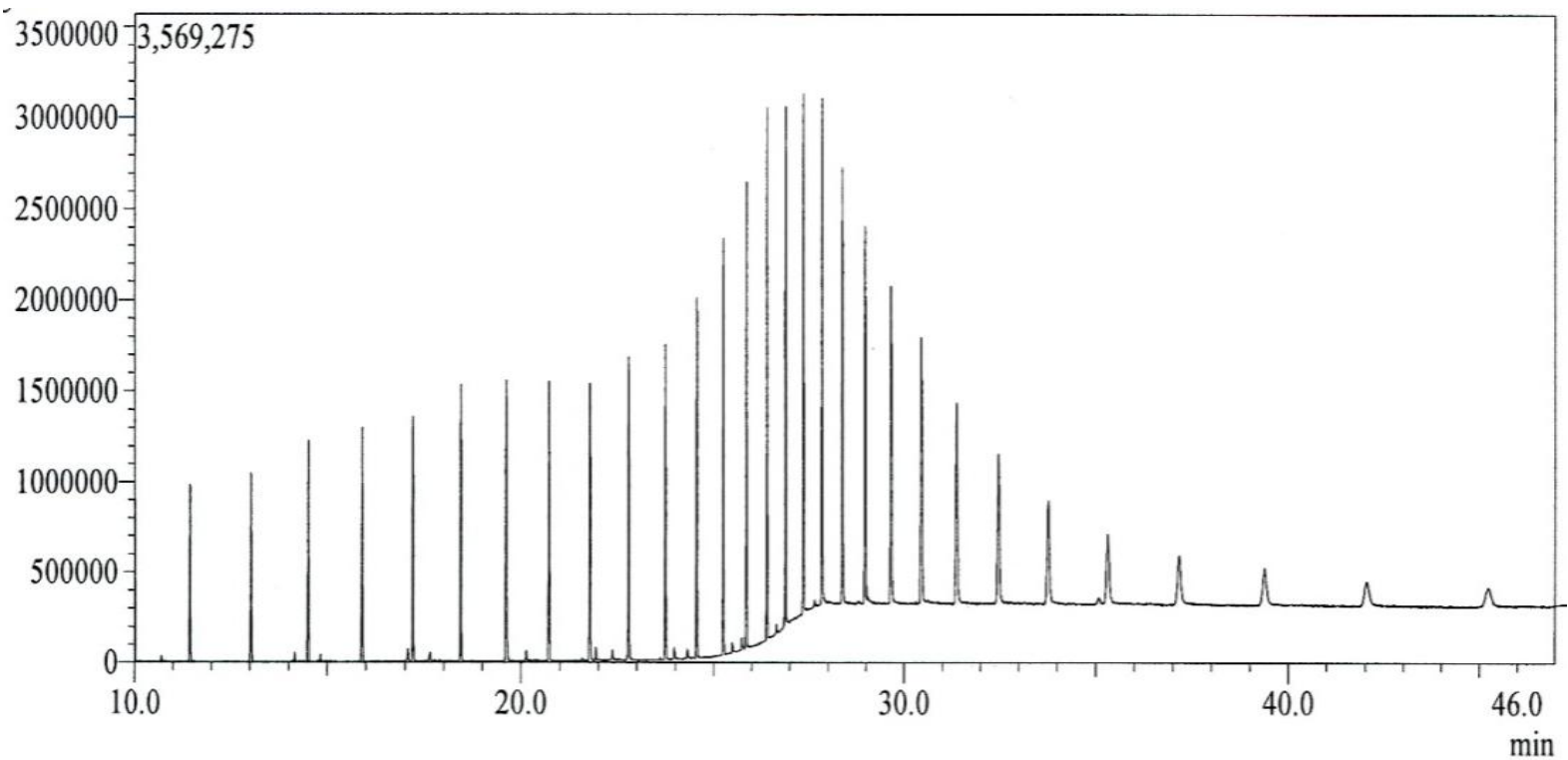

Figure 7. Hydrocarbon ladder C11-C40 on the Shimadzu GC. The elevated baseline at the end is column bleed.

The molecular ion of the first peak was used to determine the first alkane that eluted (See Figure 8). From the $\mathrm{m} / \mathrm{z}$ charge of 156 , it was determined the first alkane shown was $\mathrm{C} 11$. 


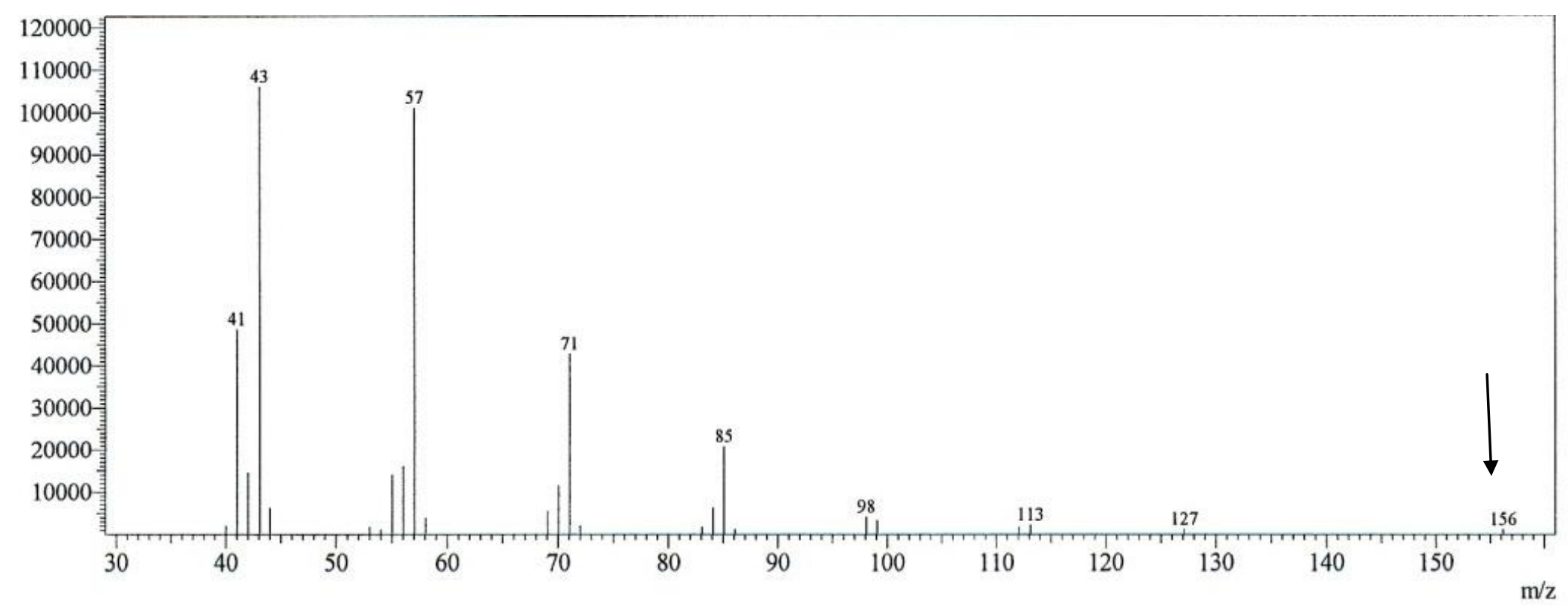

Figure 8. Mass spectrum of C11 on the Shimadzu GC. Molecular ion of $156 \mathrm{~m} / \mathrm{z}$ shown 
One consideration in calculating linear retention indices is the significant figures reported for the retention time. The retention times for the Shimadzu GC were rounded to two decimal places even though the software provided to three decimal places. This was due to the PerkinElmer GC reporting retention times to two decimal places. All the linear retention indices were eventually reported as whole number, but in order to have unity among rounding for each instrument, two decimals places were used for the retention times of each sample (prior to rounding to the whole number for the calculated linear retention index).

Five replicates of the ladder were run on the same day to determine the intra-variability using the temperature program supplied by Supleco ${ }^{\circledR}$. The $\%$ RSD of alkanes C11-C36 is smaller compared to the alkanes $\mathrm{C} 37-\mathrm{C} 40$ which are slightly larger (See Table 6). These alkanes retain in the column longer and have more time to interact with the stationary phase where cause broader peaks, which accounts for the slightly larger \%RSD. 
Table 6.Intra-day variability of retention times of 5 replicates of hydrocarbon ladder. Temperature Program supplied from Supleco ${ }^{\circledR}$.

\begin{tabular}{|c|c|c|c|c|c|c|c|c|c|c|c|c|c|c|c|}
\hline & $\mathrm{C} 11$ & $\mathrm{C} 12$ & $\mathrm{C} 13$ & $\mathrm{C} 14$ & $\mathrm{C} 15$ & $\mathrm{C} 16$ & $\mathrm{C} 17$ & C18 & C19 & $\mathrm{C} 20$ & $\mathrm{C} 21$ & $\mathrm{C} 22$ & $\mathrm{C} 23$ & $\mathrm{C} 24$ & $\mathrm{C} 25$ \\
\hline Average & 11.45 & 13.03 & 14.51 & 15.90 & 17.21 & 18.44 & 19.61 & 20.72 & 21.78 & 22.79 & 23.74 & 24.56 & 25.26 & 25.86 & 26.40 \\
\hline SD & 0.002 & 0.001 & 0.001 & 0.002 & 0.002 & 0.008 & 0.002 & 0.001 & 0.002 & 0.001 & 0.001 & 0.002 & 0.002 & 0.001 & 0.002 \\
\hline$\%$ RSD & 0.01 & 0.01 & 0.01 & 0.01 & 0.01 & 0.04 & 0.008 & 0.007 & 0.007 & 0.004 & 0.006 & 0.006 & 0.008 & 0.004 & 0.006 \\
\hline
\end{tabular}

\begin{tabular}{|c|c|c|c|c|c|c|c|c|c|c|c|c|c|c|c|}
\hline & C26 & C27 & C28 & C29 & C30 & C31 & C32 & C33 & C34 & C35 & C36 & C37 & C38 & C39 & C40 \\
\hline Average & 26.89 & 27.35 & 27.84 & 28.37 & 28.97 & 29.65 & 30.44 & 31.37 & 32.46 & 33.76 & 35.31 & 37.16 & 39.37 & 42.04 & 45.25 \\
\hline SD & 0.001 & 0.001 & 0.001 & 0.002 & 0.002 & 0.002 & 0.003 & 0.002 & 0.005 & 0.004 & 0.003 & 0.006 & 0.01 & 0.009 & 0.04 \\
\hline \%RSD & 0.004 & 0.004 & 0.005 & 0.006 & 0.007 & 0.007 & 0.01 & 0.007 & 0.02 & 0.01 & 0.008 & 0.02 & 0.03 & 0.02 & 0.09 \\
\hline
\end{tabular}


The hydrocarbon ladder was run in replicate to determine the intra-day variability using the temperature program from Method 1 on the Shimadzu GC (See Figure 9). C8-C29 eluted on the Shimadzu GC with a solvent delay of 3 minutes.

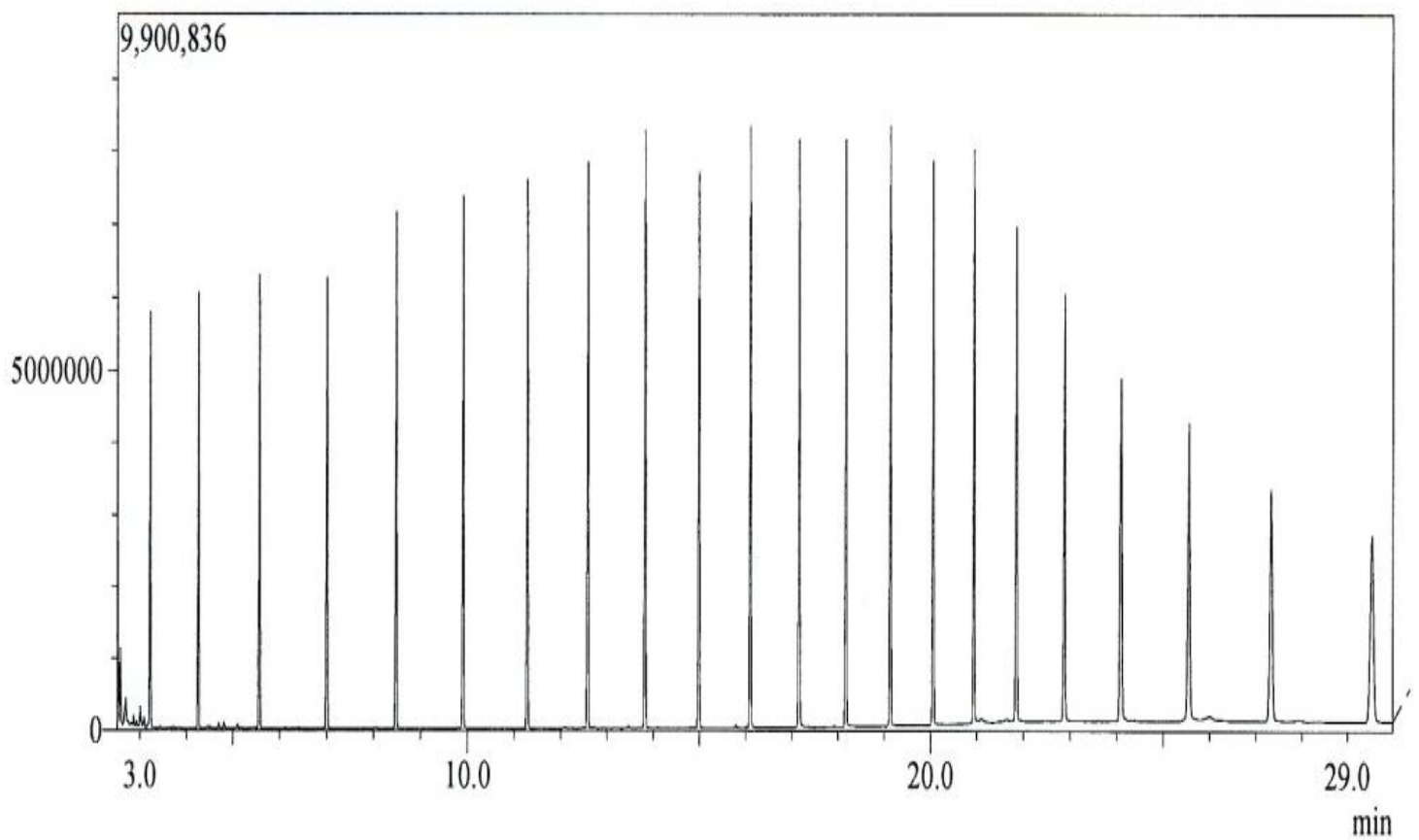

Figure 9. Hydrocarbon ladder C8-C29 on the Shimadzu GC. Temperature program used in Method 1.

The molecular ion of the first peak was used to determine the first alkane that eluted (See Figure 10). From the $\mathrm{m} / \mathrm{z}$ of 114 , it was determined the first alkane shown was $\mathrm{C} 8$. The molecular ion was seen until C24 at a m/z ratio of 338 (See Figure 11). The MS program was set to scan from $\mathrm{m} / \mathrm{z}$ of $30-350$ so the next alkane $\mathrm{m} / \mathrm{z}$ ratio was not seen. 


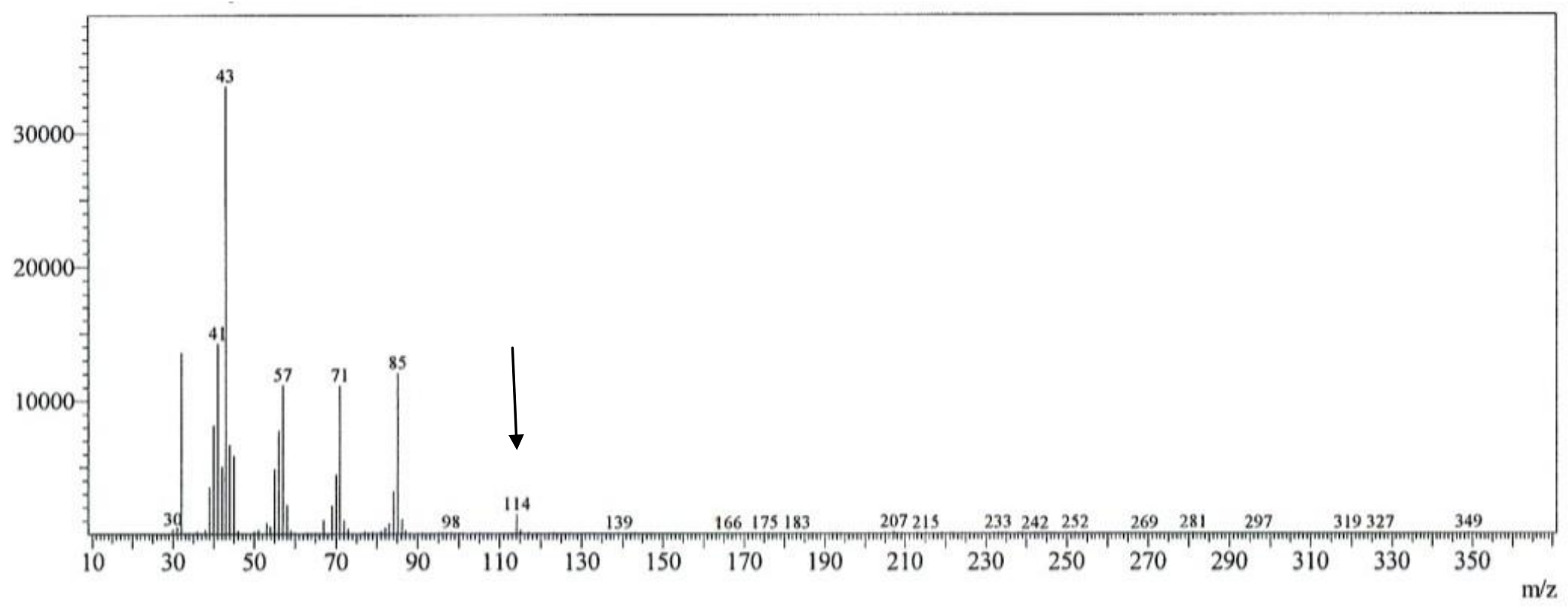

Figure 10. Mass spectrum of C8 on the Shimadzu GC. Molecular ion of $114 \mathrm{~m} / \mathrm{z}$ shown. 


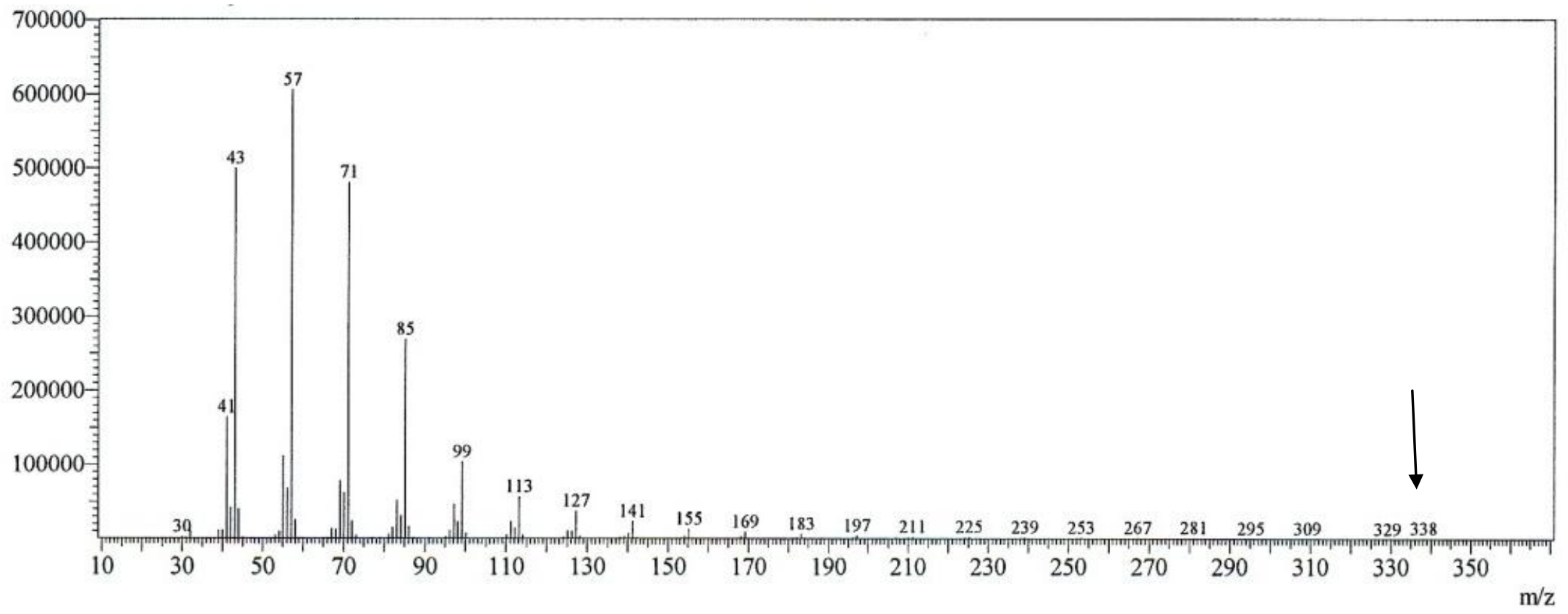

Figure 11. Mass spectrum of C24 on the Shimadzu GC. Molecular ion of $338 \mathrm{~m} / \mathrm{z}$ shown (in this spectra, the $\mathrm{m} / \mathrm{z} 338$ is very small, but present.) 
Ten replicates of the ladder were run on the same day to determine the intra-day variability using the temperature program in

Method 1 on the Shimadzu GC (See Table 7). The highest \%RSD of 0.04 was observed at C9.

Table 7. Intra-day variability of retention times of 10 replicates of the hydrocarbon ladder

\begin{tabular}{|c|c|c|c|c|c|c|c|c|c|c|c|}
\hline & C8 & C9 & C10 & C11 & C12 & C13 & C14 & C15 & C16 & C17 & C18 \\
\hline Average & 3.22 & 4.26 & 5.57 & 7.01 & 8.48 & 9.91 & 11.28 & 12.58 & 13.82 & 14.99 & 16.10 \\
\hline SD & 0.001 & 0.002 & 0.002 & 0.002 & 0.002 & 0.001 & 0.002 & 0.001 & 0.001 & 0.002 & 0.001 \\
\hline \%RSD & 0.03 & 0.04 & 0.03 & 0.03 & 0.02 & 0.01 & 0.01 & 0.007 & 0.007 & 0.01 & 0.005 \\
\hline
\end{tabular}

\begin{tabular}{|c|c|c|c|c|c|c|c|c|c|c|c|}
\hline & C19 & C20 & C21 & C22 & C23 & C24 & C25 & C26 & C27 & C28 & C29 \\
\hline Average & 17.16 & 18.17 & 19.13 & 20.05 & 20.94 & 21.84 & 22.88 & 24.09 & 25.55 & 27.33 & 29.53 \\
\hline SD & 0.001 & 0.001 & 0.001 & 0.002 & 0.001 & 0.001 & 0.002 & 0.003 & 0.004 & 0.004 & 0.004 \\
\hline \%RSD & 0.008 & 0.007 & 0.004 & 0.008 & 0.006 & 0.006 & 0.008 & 0.01 & 0.02 & 0.02 & 0.01 \\
\hline
\end{tabular}


The inter-day variability of the ladder was examined within the Shimadzu GC (See Table 8). The first day of sampling was compared to the last day of sampling, which corresponded to an elapsed time of one month. A one-way ANOVA was calculated for the inter-day variability (See Table 9). The F value is higher than the $\mathrm{F}_{\text {critical }}$ value and the $\mathrm{p}$-value was less than 0.05 , therefore the null hypothesis was rejected (stating that the means were the same on both days). The alternate hypothesis was accepted, the means are not the same between day 1 and day 5 . This is a result of the very tight distributions associated with a specific peak retention time on a single day. 
Table 8. Inter-day variability of hydrocarbon ladder compared to day 1 and day 5 of sampling

\begin{tabular}{|c|c|c|c|c|c|c|c|c|c|c|c|}
\hline & C8 & C9 & C10 & C11 & C12 & C13 & C14 & C15 & C16 & C17 & C18 \\
\hline Day 1 & 3.24 & 4.28 & 5.59 & 7.03 & 8.50 & 9.93 & 11.30 & 12.61 & 13.84 & 15.01 & 16.12 \\
\hline Day 1 & 3.24 & 4.29 & 5.59 & 7.04 & 8.50 & 9.93 & 11.31 & 12.61 & 13.84 & 15.01 & 16.12 \\
\hline Day 1 & 3.24 & 4.29 & 5.59 & 7.04 & 8.50 & 9.93 & 11.31 & 12.61 & 13.84 & 15.01 & 16.12 \\
\hline Day 5 & 3.23 & 4.27 & 5.57 & 7.01 & 8.48 & 9.91 & 11.28 & 12.58 & 13.82 & 14.99 & 16.10 \\
\hline Day 5 & 3.23 & 4.27 & 5.57 & 7.02 & 8.41 & 9.91 & 11.28 & 12.58 & 13.82 & 14.99 & 16.10 \\
\hline Day 5 & 3.23 & 4.27 & 5.57 & 7.02 & 8.48 & 9.91 & 11.28 & 12.58 & 13.82 & 14.99 & 16.10 \\
\hline & & & & & & & & & & & \\
\hline Average & 3.24 & 4.28 & 5.58 & 7.03 & 8.49 & 9.92 & 11.29 & 12.60 & 13.83 & 15.00 & 16.11 \\
\hline SD & 0.006 & 0.009 & 0.01 & 0.01 & 0.01 & 0.01 & 0.01 & 0.01 & 0.01 & 0.01 & 0.01 \\
\hline \% RSD & 0.2 & 0.2 & 0.2 & 0.2 & 0.1 & 0.1 & 0.1 & 0.1 & 0.09 & 0.08 & 0.08 \\
\hline
\end{tabular}

\begin{tabular}{|c|c|c|c|c|c|c|c|c|c|c|c|}
\hline & C19 & C20 & C21 & C22 & C23 & C24 & C25 & C26 & C27 & C28 & C29 \\
\hline Day 1 & 17.18 & 18.19 & 19.15 & 20.07 & 20.96 & 21.87 & 22.91 & 24.13 & 25.59 & 27.38 & 25.59 \\
\hline Day 1 & 17.18 & 18.19 & 19.15 & 20.07 & 20.96 & 21.87 & 22.91 & 24.12 & 25.59 & 27.38 & 29.59 \\
\hline Day 1 & 17.18 & 18.19 & 19.15 & 20.08 & 20.96 & 21.86 & 22.90 & 24.12 & 25.59 & 27.37 & 29.58 \\
\hline Day 5 & 17.16 & 18.17 & 19.13 & 20.05 & 20.93 & 21.84 & 22.87 & 24.09 & 25.55 & 27.32 & 29.52 \\
\hline Day 5 & 17.16 & 18.17 & 19.13 & 20.05 & 20.93 & 21.84 & 22.87 & 24.09 & 25.54 & 27.33 & 29.52 \\
\hline Day 5 & 17.16 & 18.17 & 19.13 & 20.05 & 20.93 & 21.84 & 22.87 & 24.09 & 25.55 & 27.33 & 29.52 \\
\hline & & & & & & & & & & & \\
\hline Average & 17.17 & 18.18 & 19.14 & 20.06 & 20.94 & 21.85 & 22.89 & 24.10 & 25.57 & 27.35 & 29.55 \\
\hline SD & 0.01 & 0.01 & 0.01 & 0.01 & 0.01 & 0.01 & 0.02 & 0.02 & 0.02 & 0.03 & 0.04 \\
\hline \% RSD & 0.07 & 0.07 & 0.06 & 0.06 & 0.06 & 0.07 & 0.07 & 0.09 & 0.09 & 0.1 & 0.1 \\
\hline
\end{tabular}


Table 9. One-way ANOVA table for the inter-day variability of the hydrocarbon ladders on the Shimadzu GC

\begin{tabular}{|c|c|c|c|c|c|c|}
\hline $\begin{array}{c}\text { Source of } \\
\text { Variation }\end{array}$ & SS & df & MS & F & P-value & F crit \\
\hline $\begin{array}{c}\text { Between } \\
\text { Groups }\end{array}$ & 7382.57 & 21 & 351.55 & $\mathbf{1 . 3 1 \times 1 \mathbf { 1 0 } ^ { \mathbf { 6 } }}$ & $<\mathbf{1 0}^{-\mathbf{2 0 0}}$ & $\mathbf{1 . 6 5}$ \\
\hline $\begin{array}{c}\text { Within } \\
\text { Groups }\end{array}$ & 0.029 & 110 & 0.00027 & & & \\
\hline Total & 7382.60 & 131 & & & & \\
\hline
\end{tabular}




\section{Hydrocarbon ladder-PerkinElmer GC}

Alkanes C9-C33 eluted on the PerkinElmer GC using a solvent delay of with a solvent delay of 2.5 minutes (See Figure 12). This ladder was run in replicates in 24 replicates to ensure repeatability. The temperature program used can be seen in Method 2.

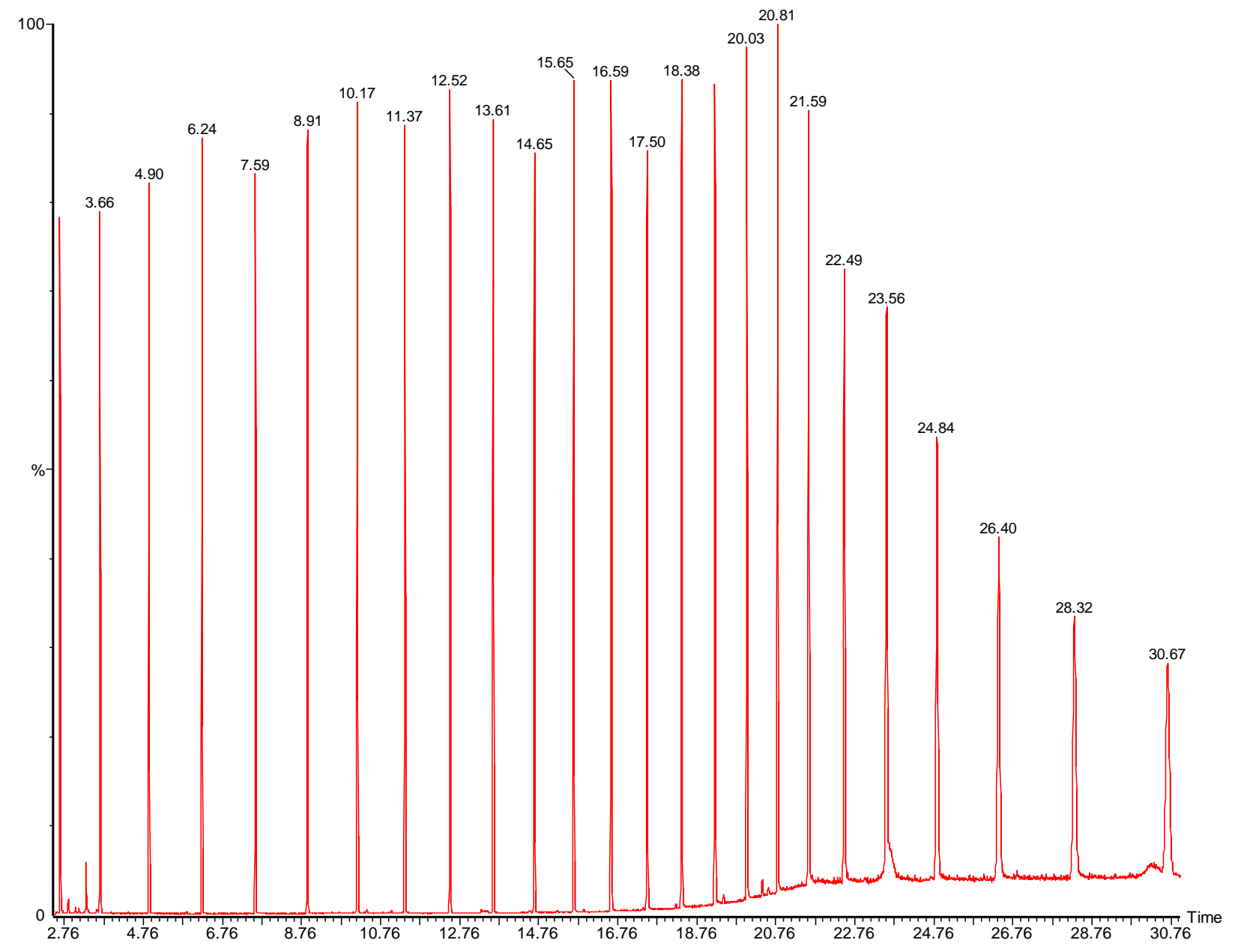

Figure 12. Hydrocarbon ladder C9-C33 on the PerkinElmer GC.

The molecular ion of the first peak was used to determine the first alkane that eluted (See Figure 13). From the $\mathrm{m} / \mathrm{z}$ charge of 128 , it was determined the first alkane shown was $\mathrm{C} 10$. The molecular ion was seen until C24 at a m/z ratio of 338 (See Figure 14). 


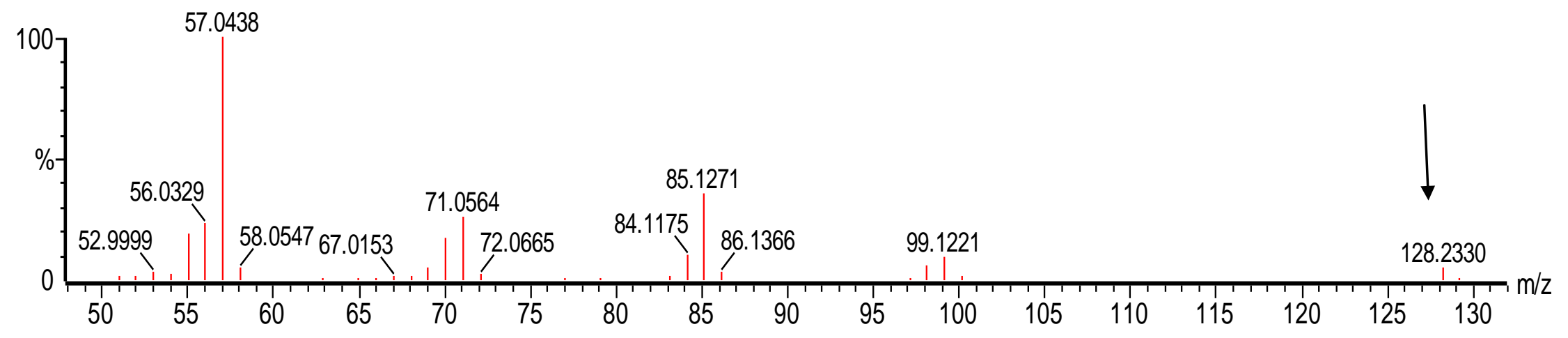

Figure 13. Mass spectrum of C7 on the PerkinElmer GC. Molecular ion of $128 \mathrm{~m} / \mathrm{z}$ is shown

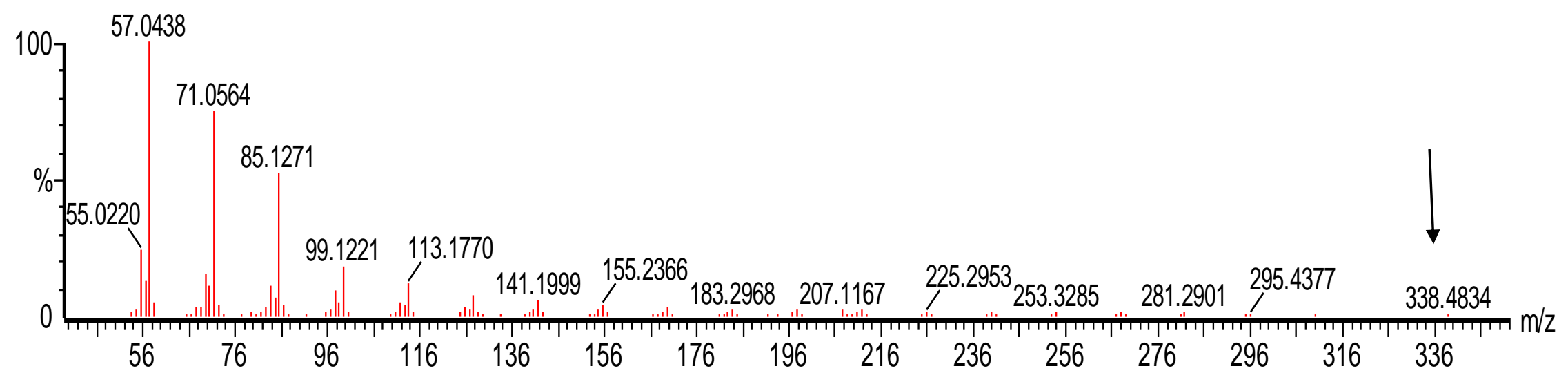

Figure 14. Mass spectrum of C24 on the PerkinElmer GC. Molecular ion of $338 \mathrm{~m} / \mathrm{z}$ is shown (in this spectra, the $\mathrm{m} / \mathrm{z} 338$ is very small, but present.) 
The molecular ion of the first peak was used to determine the first alkane that eluted with a solvent delay of 3 minutes. From the $\mathrm{m} / \mathrm{z}$ charge of 128 , it was determined the first alkane shown was C10. The molecular ion was seen until C24 at a m/z ratio of 338 (See Figure 14).

Since the Shimadzu GC had a solvent delay of 3 minutes, the PerkinElmer GC was changed to a solvent delay of 3 minutes. Alkanes C10-C33 eluted on the PerkinElmer GC using a solvent delay of 3minutes (See Figure 15). All of the drug standards fell within this range; therefore this solvent delay was did not influence the linear retention index calculations. This ladder was run in replicates of ten to ensure repeatability. The temperature program used can be seen in Method 1. 


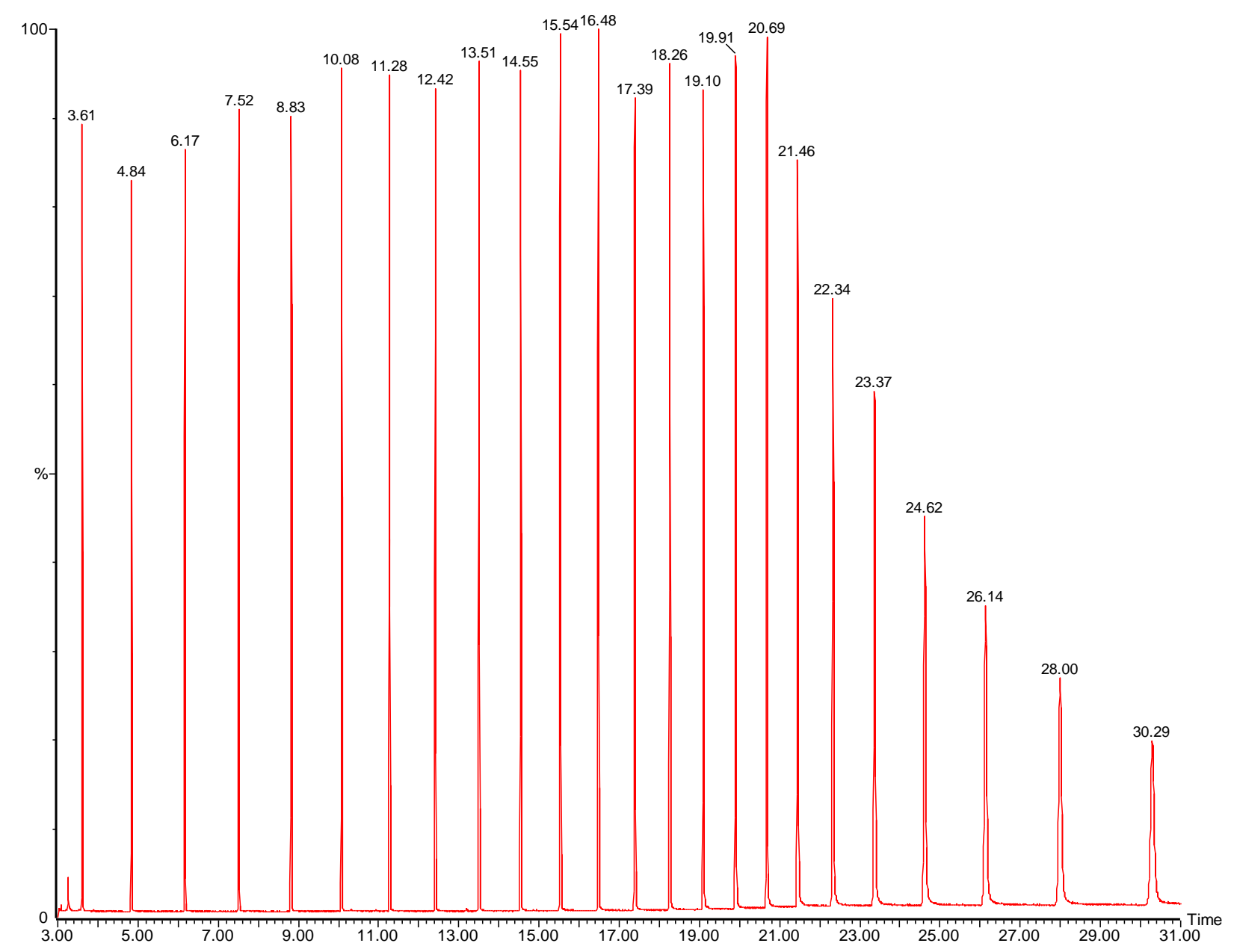

Figure 15. Hydrocarbon ladder C10-C33 on the PerkinElmer GC. Temperature program used in Method 2.

The molecular ion of the first peak was used to determine the first alkane that eluted (See Figure 16) with a solvent delay of 3 minutes. From the $\mathrm{m} / \mathrm{z}$ charge of 142 , it was determined the first alkane shown was C10. The molecular ion was seen until C24 at a m/z ratio of 338 (See Figure 17). 


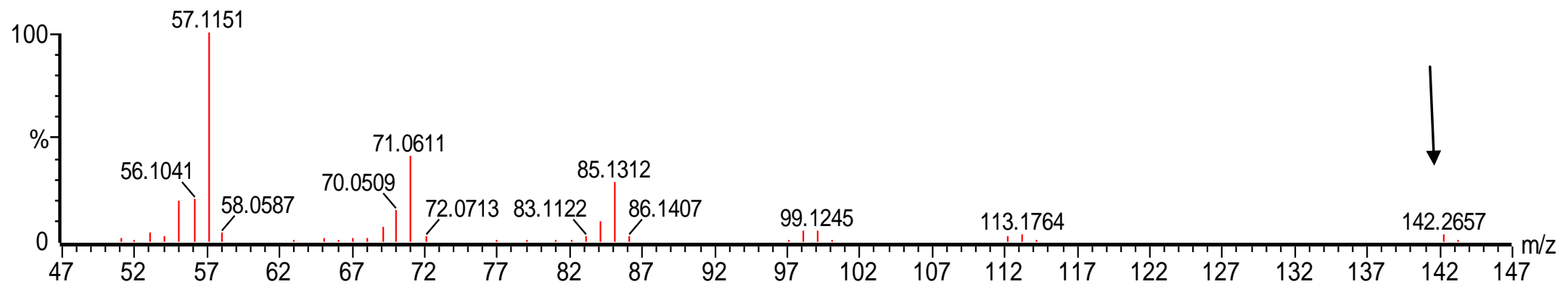

Figure 16. Mass spectrum of C10 on the PerkinElmer GC. Used Molecular ion of $142 \mathrm{~m} / \mathrm{z}$ is shown.

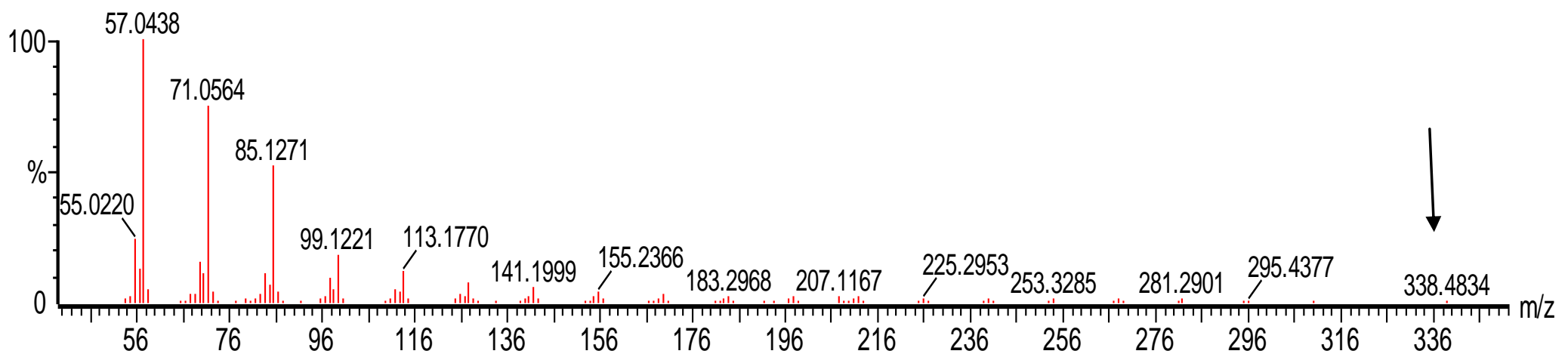

Figure 17. Mass spectrum of C24 on the PerkinElmer GC. Used Molecular ion of $338 \mathrm{~m} / \mathrm{z}$ is shown (in this spectra, the $\mathrm{m} / \mathrm{z} 338$ is very small, but present.) 
Twenty-four replicates of the ladder were run on the same day to determine the intra-variability using the temperature program in Method 1 (See Table 10). The largest \%RSD was seen at C9 at a value of 0.306.

Table 10. Intra-day variability of retention times of 24 replicates of hydrocarbon ladder using Method 2 parameters.

\begin{tabular}{|c|c|c|c|c|c|c|c|c|c|c|c|c|c|}
\hline & C9 & $\mathrm{C} 10$ & $\mathrm{C} 11$ & $\mathrm{C} 12$ & $\mathrm{C} 13$ & C14 & $\mathrm{C} 15$ & $\mathrm{C} 16$ & C17 & C18 & C19 & $\mathrm{C} 20$ & $\mathrm{C} 21$ \\
\hline Average & 2.67 & 3.69 & 4.93 & 6.26 & 7.61 & 8.92 & 10.18 & 11.38 & 12.53 & 13.62 & 14.65 & 15.65 & 16.60 \\
\hline SD & 0.008 & 0.009 & 0.009 & 0.008 & 0.008 & 0.006 & 0.006 & 0.006 & 0.005 & 0.005 & 0.01 & 0.008 & 0.006 \\
\hline$\%$ RSD & 0.3 & 0.2 & 0.2 & 0.1 & 0.1 & 0.07 & 0.06 & 0.05 & 0.04 & 0.04 & 0.07 & 0.05 & 0.03 \\
\hline
\end{tabular}

\begin{tabular}{|c|c|c|c|c|c|c|c|c|c|c|c|c|}
\hline & $\mathrm{C} 23$ & $\mathrm{C} 22$ & $\mathrm{C} 24$ & $\mathrm{C} 25$ & C26 & C27 & C28 & $\mathrm{C} 29$ & C30 & C31 & $\mathrm{C} 22$ & $\mathrm{C} 23$ \\
\hline Average & 18.38 & 17.51 & 19.22 & 20.03 & 20.80 & 21.58 & 22.49 & 23.55 & 24.84 & 26.39 & 28.30 & 30.67 \\
\hline SD & 0.005 & 0.005 & 0.005 & 0.005 & 0.008 & 0.008 & 0.007 & 0.008 & 0.03 & 0.01 & 0.01 & 0.01 \\
\hline$\%$ RSD & 0.03 & 0.03 & 0.03 & 0.03 & 0.04 & 0.04 & 0.03 & 0.03 & 0.11 & 0.04 & 0.04 & 0.04 \\
\hline
\end{tabular}


The inter-day variability of the ladder was examined within the PerkinElmer GC (See Table 11). The first day of sampling was compared to the last day of sampling. The time in between these two days of sampling is approximately one month. A one-way ANOVA was calculated for the inter-day variability between day 1 and day 5 of sampling (See Table 12). The F value is more than the $F_{\text {critical }}$ value and the p-value is less than 0.05 , indicating that the null hypothesis is rejected, the means of the two days are not the same. 
Table 11. Inter-day variability of hydrocarbon ladder compared to day 1 and day 5 of sampling on the PerkinElmer GC

\begin{tabular}{|c|c|c|c|c|c|c|c|c|c|c|c|c|}
\hline & C10 & C11 & $\mathrm{C} 12$ & C13 & $\mathrm{C} 14$ & C15 & C16 & C17 & C18 & C19 & $\mathrm{C} 20$ & $\mathrm{C} 21$ \\
\hline Day 1 & 3.62 & 4.85 & 6.17 & 7.52 & 8.83 & 10.09 & 11.28 & 12.42 & 13.51 & 14.55 & 15.54 & 16.49 \\
\hline Day 1 & 3.62 & 4.84 & 6.17 & 7.52 & 8.83 & 10.08 & 11.28 & 12.42 & 13.51 & 14.55 & 15.54 & 16.48 \\
\hline Day1 & 3.62 & 4.85 & 6.18 & 7.53 & 8.83 & 10.09 & 11.29 & 12.43 & 13.51 & 14.55 & 15.54 & 16.49 \\
\hline Day 5 & 3.61 & 4.84 & 6.17 & 7.52 & 8.82 & 10.08 & 11.28 & 12.42 & 13.5 & 14.54 & 15.54 & 16.48 \\
\hline Day 5 & 3.62 & 4.85 & 6.17 & 7.52 & 8.83 & 10.08 & 11.28 & 12.42 & 13.51 & 14.55 & 15.54 & 16.49 \\
\hline Day 5 & 3.62 & 4.85 & 6.18 & 7.52 & 8.83 & 10.09 & 11.28 & 12.42 & 13.51 & 14.55 & 15.54 & 16.49 \\
\hline Average & 3.62 & 4.85 & 6.17 & 7.52 & 8.83 & 10.09 & 11.28 & 12.42 & 13.51 & 14.55 & 15.54 & 16.49 \\
\hline SD & 0.00 & 0.01 & 0.01 & 0.00 & 0.00 & 0.01 & 0.00 & 0.00 & 0.00 & 0.00 & 0.00 & 0.01 \\
\hline$\% \mathrm{RSD}$ & 0.1 & 0.1 & 0.08 & 0.05 & 0.05 & 0.05 & 0.04 & 0.03 & 0.03 & 0.03 & 0.00 & 0.03 \\
\hline
\end{tabular}

\begin{tabular}{|c|c|c|c|c|c|c|c|c|c|c|c|c|}
\hline & $\mathrm{C} 22$ & $\mathrm{C} 23$ & C24 & $\mathrm{C} 25$ & $\mathrm{C} 26$ & $\mathrm{C} 27$ & $\mathrm{C} 28$ & C29 & C30 & C31 & C32 & C33 \\
\hline Day 1 & 17.40 & 18.27 & 19.11 & 19.91 & 20.69 & 21.46 & 22.34 & 23.38 & 24.63 & 26.15 & 28.01 & 30.31 \\
\hline Day 1 & 17.39 & 18.26 & 19.10 & 19.91 & 20.69 & 21.46 & 22.34 & 23.38 & 24.62 & 26.15 & 28.00 & 30.30 \\
\hline Day1 & 17.40 & 18.27 & 19.11 & 19.92 & 20.69 & 21.46 & 22.34 & 23.38 & 24.63 & 26.14 & 28.01 & 30.30 \\
\hline Day 5 & 17.39 & 18.26 & 19.10 & 19.91 & 20.68 & 21.45 & 22.33 & 23.37 & 24.62 & 26.14 & 28.00 & 30.30 \\
\hline Day 5 & 17.39 & 18.26 & 19.10 & 19.91 & 20.69 & 21.45 & 22.34 & 23.37 & 24.62 & 26.14 & 28.00 & 30.30 \\
\hline Day 5 & 17.39 & 18.27 & 19.10 & 19.91 & 20.69 & 21.46 & 22.34 & 23.37 & 24.62 & 26.14 & 28.00 & 30.31 \\
\hline Average & 17.39 & 18.27 & 19.10 & 19.91 & 20.69 & 21.46 & 22.34 & 23.38 & 24.62 & 26.14 & 28.00 & 30.30 \\
\hline SD & 0.01 & 0.01 & 0.01 & 0.00 & 0.00 & 0.01 & 0.00 & 0.01 & 0.01 & 0.01 & 0.01 & 0.01 \\
\hline$\%$ RSD & 0.03 & 0.03 & 0.03 & 0.02 & 0.02 & 0.02 & 0.02 & 0.02 & 0.02 & 0.02 & 0.02 & 0.02 \\
\hline
\end{tabular}


Table 12. One-way ANOVA table for the inter-day variability of the hydrocarbon ladders on the PerkinElmer GC

\begin{tabular}{|c|c|c|c|c|c|c|}
\hline $\begin{array}{l}\text { Source of } \\
\text { Variation }\end{array}$ & SS & df & MS & $\mathrm{F}$ & P-value & F crit \\
\hline $\begin{array}{c}\text { Between } \\
\text { Groups }\end{array}$ & 7644.98 & 23 & 332.39 & $1.52 \times 10^{7}$ & $\mathbf{0}$ & 1.62 \\
\hline $\begin{array}{l}\text { Within } \\
\text { Groups }\end{array}$ & 0.0026 & 120 & $2.18 \times 10^{-05}$ & & & \\
\hline Total & 7644.99 & 143 & & & & \\
\hline
\end{tabular}




\section{Hydrocarbon ladder-PerkinElmer GC (modified temperature program from Method 3)}

Since the experimental conditions were not identical in Method 1 and Method 2 between the two instruments, the ladder and drug standards were run again using the conditions in Method 3. The experimental parameters were the same and alkanes C10-C33 eluted (See Figure 18). The peaks were identified using the $\mathrm{m} / \mathrm{z}$ ratio of each peak. It was determined the first peak was $\mathrm{C} 10$ (See Figure 19) and the last alkane shown was for C24 by their $\mathrm{m} / \mathrm{z}$ ratio (See Figure 20). This corresponds to the ladder run with the temperature program 6 for the PerkinElmer.

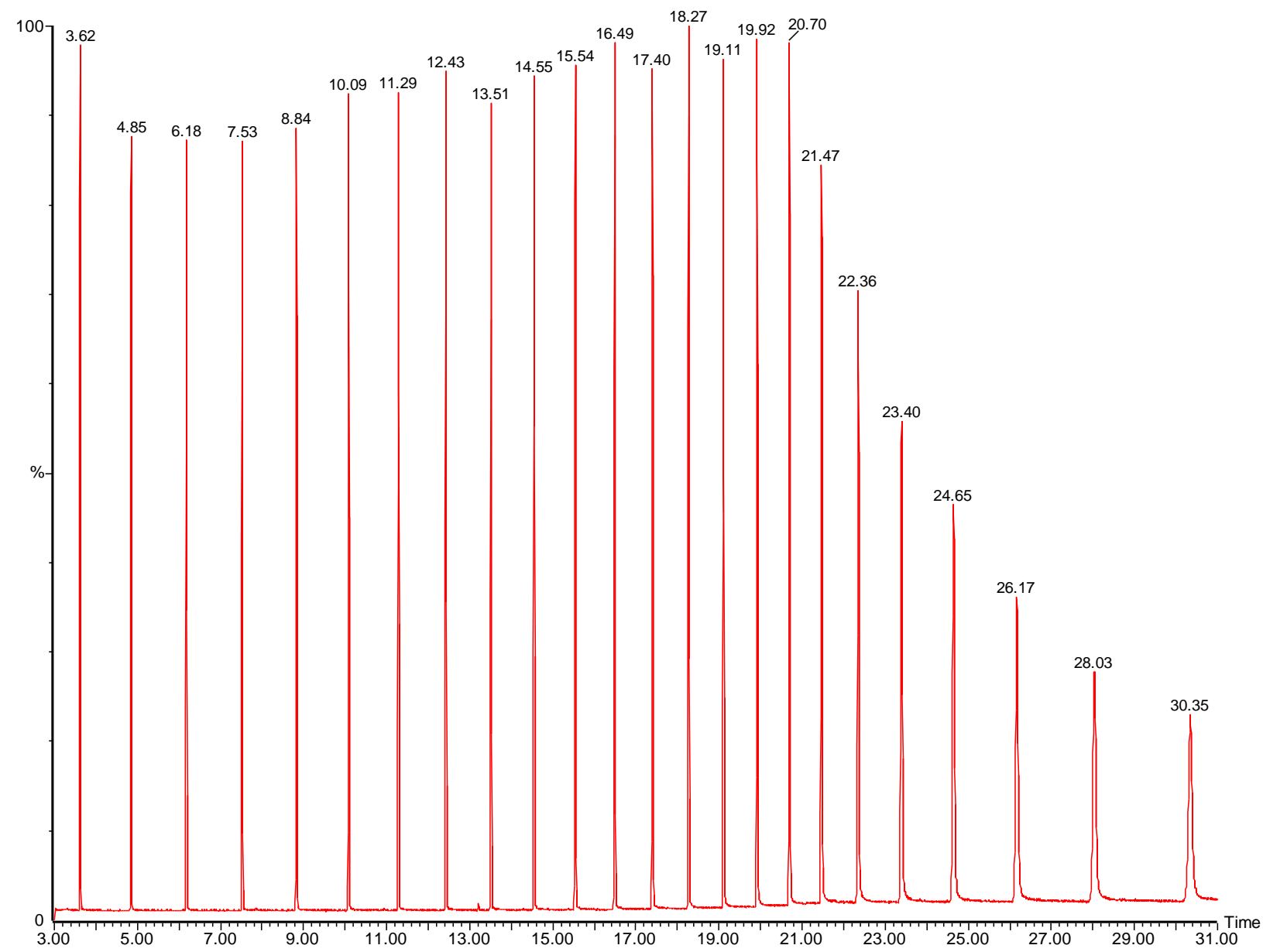

Figure 18. Hydrocarbon ladder C10-C33 on the PerkinElmer GC. Temperature program used in Method 3. 


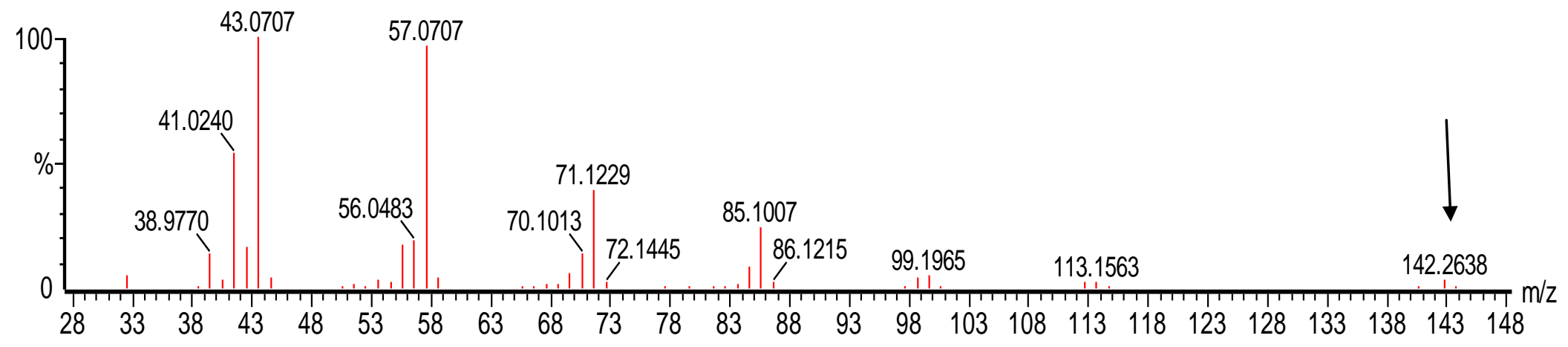

Figure 19. Mass spectrum of C10 on the PerkinElmer GC. Used Molecular ion of $142 \mathrm{~m} / \mathrm{z}$ to identify C10.

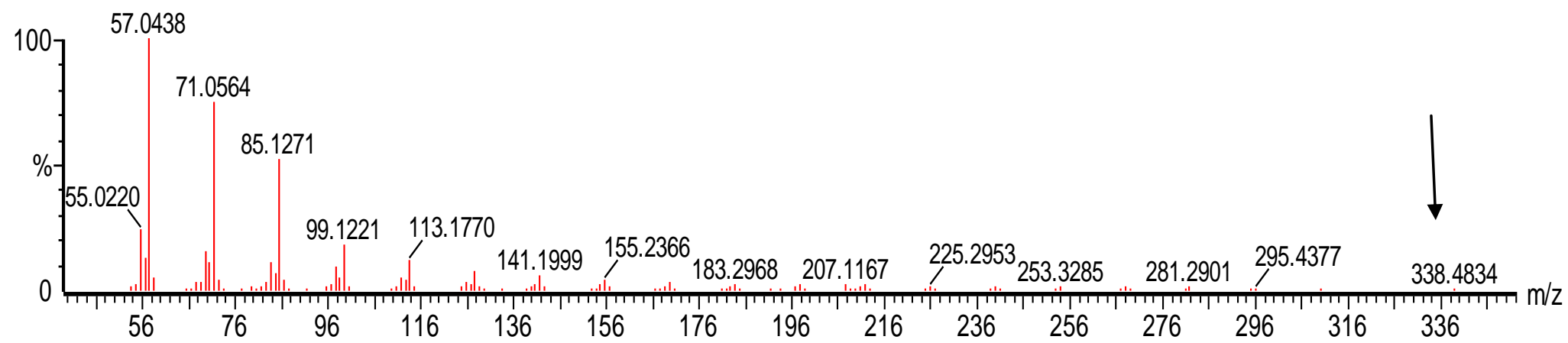

Figure 20. Mass spectrum of C24 on the PerkinElmer GC. Used Molecular ion of $338 \mathrm{~m} / \mathrm{z}$ to identify C24. (in this spectra, the m/z 338 is very small, but present.) 
Three replicates of the ladder were run on the same day to determine the intra-variability using the temperature program in Method 3 (See Table 13). The parameters that were changed were the injection port temperature and the interface temperature to be consistent with the Shimadzu GC/MS parameters from Method 1.

A hypothesis test (student's t-test) was applied for comparison between the 15 replicates of the ladder with the temperature program from Method 2 to the three replicates from the modified temperature program from Method 3. Since the data is unbalanced, ANOVA could not be applied. For the student's t-test, if the t table value was higher than 5\% (marked in bold- See Table 14), the null hypothesis was rejected. Only the values that had some deviation to the mean could be compared (alkanes that did not have any deviation are marked "-“). Three alkanes (C14, C22, and C24) had a higher $\mathrm{t}$ table value of 0.05 these values cannot be combined with the other data set. However, these three alkanes were not used to calculate the LRI of any of the 9 drugs, they did not influence the calculation of LRI.

A paired t-test was applied to 3 of the 15 replicates from the temperature program of Method 2 and the 3 replicates of the modified temperature program from Method 3 (See Table 15). For the paired $t$-test, if the $t$ table value was higher than 0.05 , the null hypothesis was rejected meaning there was not a significant difference between the retention times of the alkanes. The paired t-test showed that alkanes C29-C33 were significantly different $(\mathrm{p}<0.05$, marked in bold) while the other alkanes were not significantly different. The alkanes that eluted later showed a slightly larger variation compared to the rest of the alkanes. Also, the retention times of the alkanes from the modified temperature program were slightly longer of about 0.01 minutes compared to the retention times of the ladder using Method 2. 
Table 13. Intra-day variability of retention times of 3 replicates of the hydrocarbon ladder using Method 3 parameters.

\begin{tabular}{|c|c|c|c|c|c|c|c|c|c|c|c|c|}
\hline & $\mathrm{C} 10$ & $\mathrm{C} 11$ & $\mathrm{C} 12$ & $\mathrm{C} 13$ & $\mathrm{C} 14$ & $\mathrm{C} 15$ & $\mathrm{C} 16$ & $\mathrm{C} 17$ & $\mathrm{C} 18$ & $\mathrm{C} 19$ & $\mathrm{C} 20$ & $\mathrm{C} 21$ \\
\hline Average & 3.62 & 4.85 & 6.18 & 7.53 & 8.83 & 10.09 & 11.29 & 12.43 & 13.51 & 14.55 & 15.54 & 16.49 \\
\hline SD & 0.00 & 0.00 & 0.00 & 0.01 & 0.01 & 0.00 & 0.01 & 0.00 & 0.00 & 0.00 & 0.00 & 0.00 \\
\hline \%RSD & 0.00 & 0.00 & 0.00 & 0.08 & 0.07 & 0.00 & 0.05 & 0.00 & 0.00 & 0.00 & 0.00 & 0.00 \\
\hline
\end{tabular}

\begin{tabular}{|c|c|c|c|c|c|c|c|c|c|c|c|c|}
\hline & C22 & C23 & C24 & C25 & C26 & C27 & C28 & C29 & C30 & C31 & C32 & C33 \\
\hline Average & 17.40 & 18.27 & 19.11 & 19.92 & 20.69 & 21.46 & 22.35 & 23.39 & 24.64 & 26.16 & 28.03 & 30.33 \\
\hline SD & 0.01 & 0.00 & 0.01 & 0.00 & 0.01 & 0.01 & 0.01 & 0.01 & 0.01 & 0.01 & 0.00 & 0.02 \\
\hline \%RSD & 0.03 & 0.00 & 0.03 & 0.00 & 0.03 & 0.03 & 0.03 & 0.02 & 0.02 & 0.04 & 0.00 & 0.05 \\
\hline
\end{tabular}


Table 14. Student t-test at 95\% confidence interval for hydrocarbon ladder comparing Method 2 and Method 3 parameters.

\begin{tabular}{|c|c|c|c|c|c|c|c|c|c|c|c|c|}
\hline Alkane & $\mathrm{C} 10$ & C11 & $\mathrm{C} 12$ & $\mathrm{C} 13$ & C14 & C15 & C16 & C17 & C18 & C19 & C20 & $\mathrm{C} 21$ \\
\hline $\begin{array}{c}\text { T Table } \\
\text { value } \\
95 \%\end{array}$ & -- & -- & -- & 0.02 & 0.05 & -- & 0.01 & -- & -- & -- & -- & -- \\
\hline
\end{tabular}

\begin{tabular}{|c|c|c|c|c|c|c|c|c|c|c|c|c|}
\hline Alkane & $\mathrm{C} 22$ & $\mathrm{C} 23$ & $\mathrm{C} 24$ & $\mathrm{C} 25$ & C26 & $\mathrm{C} 27$ & $\mathrm{C} 28$ & $\mathrm{C} 29$ & C30 & C31 & C32 & C33 \\
\hline $\begin{array}{c}\text { T Table } \\
\text { value } \\
95 \%\end{array}$ & 0.06 & -- & 0.12 & -- & 0.00 & 0.03 & 0.00 & 0.00 & 0.00 & 0.00 & -- & 0.00 \\
\hline
\end{tabular}

Table 15. Paired t-test at 95\% confidence interval for hydrocarbon ladder comparing Method 2 and Method 3 parameters

\begin{tabular}{|c|c|c|c|c|c|c|c|c|c|c|c|c|}
\hline Alkane & $\mathrm{C} 10$ & $\mathrm{C} 11$ & $\mathrm{C} 12$ & $\mathrm{C} 13$ & C14 & $\mathrm{C} 15$ & $\mathrm{C} 16$ & C17 & $\mathrm{C} 18$ & C19 & $\mathrm{C} 20$ & $\mathrm{C} 21$ \\
\hline $\begin{array}{c}\text { T Table } \\
\text { value } \\
95 \%\end{array}$ & 0.4 & 0.4 & 0.2 & 0.2 & 0.4 & 0.2 & 0.2 & 0.2 & 0.4 & 0.4 & 0.4 & 0.4 \\
\hline
\end{tabular}

\begin{tabular}{|c|c|c|c|c|c|c|c|c|c|c|c|c|}
\hline Alkane & $\mathrm{C} 22$ & $\mathrm{C} 23$ & $\mathrm{C} 24$ & $\mathrm{C} 25$ & $\mathrm{C} 26$ & $\mathrm{C} 27$ & $\mathrm{C} 28$ & $\mathrm{C} 29$ & C30 & C31 & C32 & C33 \\
\hline $\begin{array}{c}\text { T Table } \\
\text { value } \\
95 \%\end{array}$ & 0.2 & 0.2 & 0.2 & 0.2 & 0.5 & 0.2 & 0.1 & 0.02 & 0.02 & 0.04 & 0.02 & 0.01 \\
\hline
\end{tabular}




\section{Linear retention index Data- Shimadzu GC}

Each data set contained three replicates of the hydrocarbon ladder and three replicates of each of the nine drug standards. The ladder retention times were averaged for the three runs and used to calculate LRI for each set of data. The retention index of each drug was calculated three times (one per each retention time for each drug) and then averaged (See Table 16). The averaged LRI is seen below in the table.

Table 16. Combined linear retention index data on the Shimadzu GC. Each Day three replicates were done. Average of the retention indices are shown. Note that six significant figures were retained as per standard rules of rounding even though RIs are typically reported to whole numbers.

\begin{tabular}{|l|c|c|c|c|c|}
\hline Drug Samples & Day 1 & Day 2 & Day 3 & Day 4 & Day 5 \\
\hline Methamphetamine & 1192.90 & 1193.39 & $\mathbf{1 2 0 7 . 2 3}$ & 1193.51 & 1193.34 \\
\hline D-Amphetamine & 1137.99 & 1141.49 & 1144.06 & 1141.46 & 1139.49 \\
\hline Phentermine & $\mathbf{1 1 9 1 . 8 0}$ & $\mathbf{1 1 9 3 . 1 9}$ & $\mathbf{1 1 8 8 . 6 4}$ & 1175.12 & 1174.49 \\
\hline Pentobarbital & 1764.87 & 1765.65 & 1765.80 & 1767.27 & 1766.76 \\
\hline Butabarbital & 1686.27 & 1687.24 & 1687.55 & 1688.95 & 1688.42 \\
\hline Phenobarbital & 2006.44 & 2007.89 & 2007.72 & 2008.07 & 2007.30 \\
\hline Heroin & 2707.75 & 2709.28 & 2709.31 & 2710.33 & 2709.32 \\
\hline Oxycodone & 2623.96 & 2625.96 & 2626.31 & 2627.44 & 2626.32 \\
\hline Codeine & 2477.42 & 2479.61 & 2479.43 & 2480.54 & 2479.97 \\
\hline
\end{tabular}

The first two runs of methamphetamine and phentermine yielded LRI values that are more similar than expected. The third run showed a larger difference between the two which was more expected. The same samples were used for the first two runs on the Shimadzu GC and on the PerkinElmer GC. The LRI of methamphetamine and phentermine were about 20 riu apart each day that was evaluated (100 ppm). Peak fronting was exhibited at $100 \mathrm{ppm}$ in methamphetamine (See Figure 21) and phentermine (See Figure 23) in the Shimadzu GC, which accounted for the smaller difference in LRI between the two. The values in bold are the values where the peak fronting was exhibited. A thicker film thickness has a higher mass transfer term according to the van Deemter equation. Since there is a higher capacity for interactions between 
the analyte and the thicker stationary phase, generally a higher concentration can be efficiently separated using a thicker film. However, in the case of methamphetamine and phentermine, a higher concentration caused the column to overload and influenced the retention times which affected the linear retention index. The concentrations were lowered to $10 \mathrm{ppm}$ to avoid peak fronting both drugs (See Figure 22 and 24).

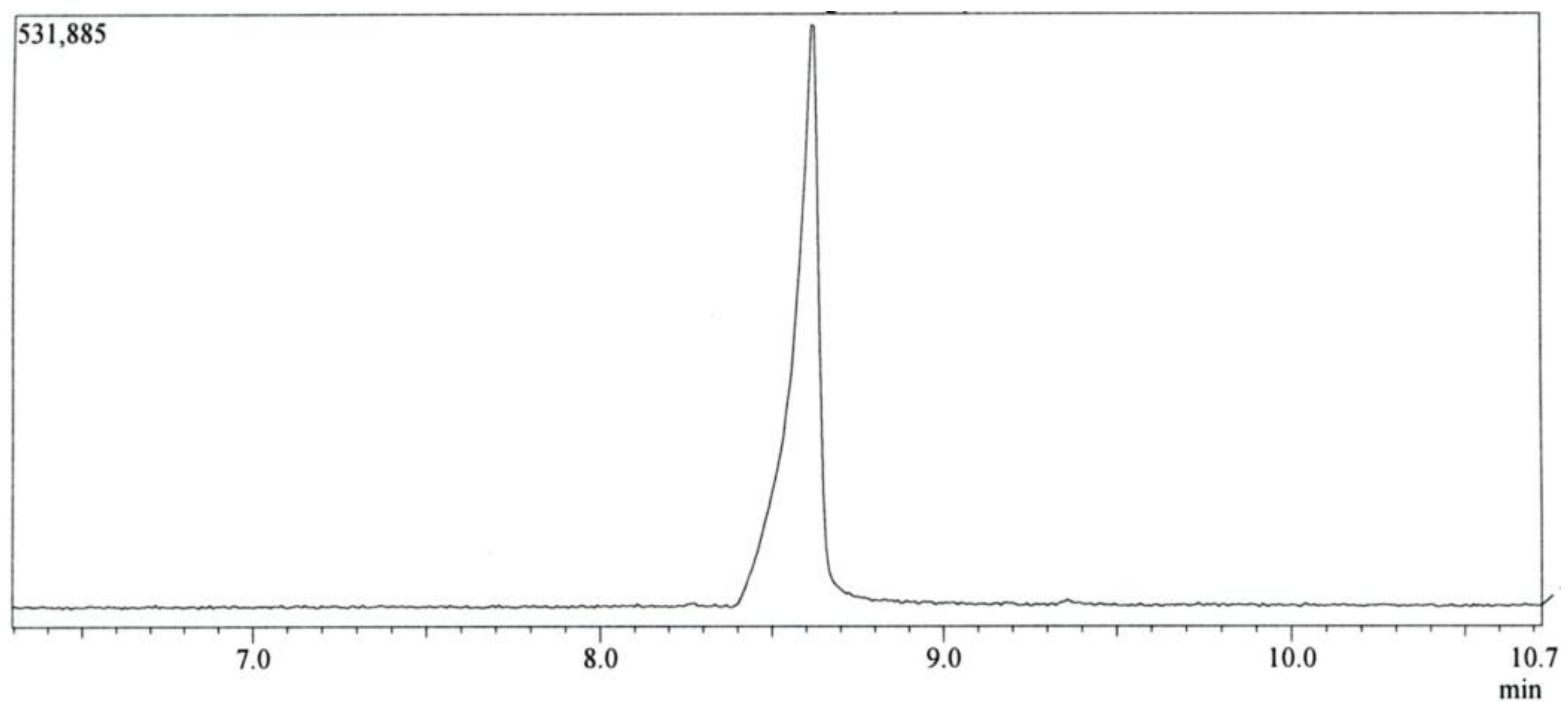

Figure 21. Methamphetamine chromatograph at 100 ppm. Showed signs of peak fronting which is an indication of overloading 


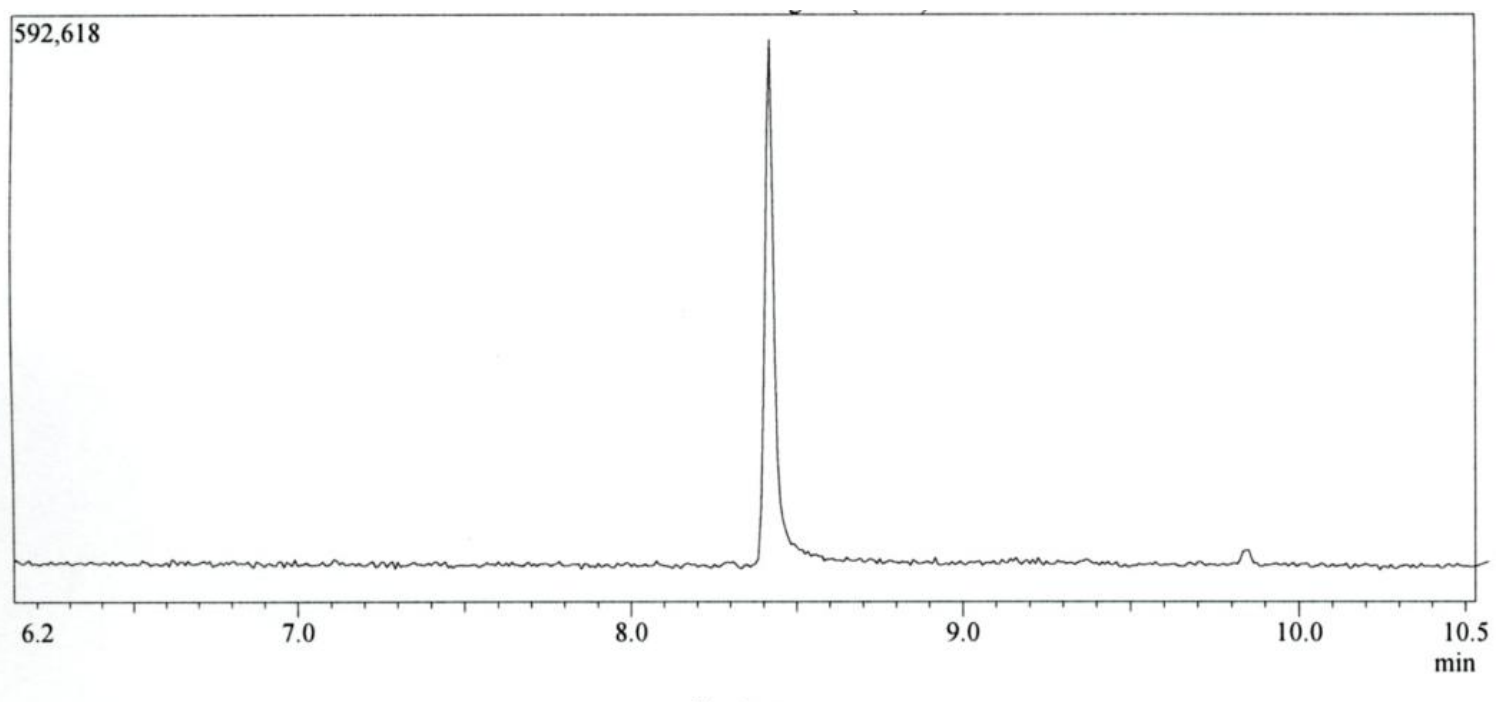

Figure 22. Methamphetamine chromatograph at 10 ppm. Not showing signs of peak fronting. 


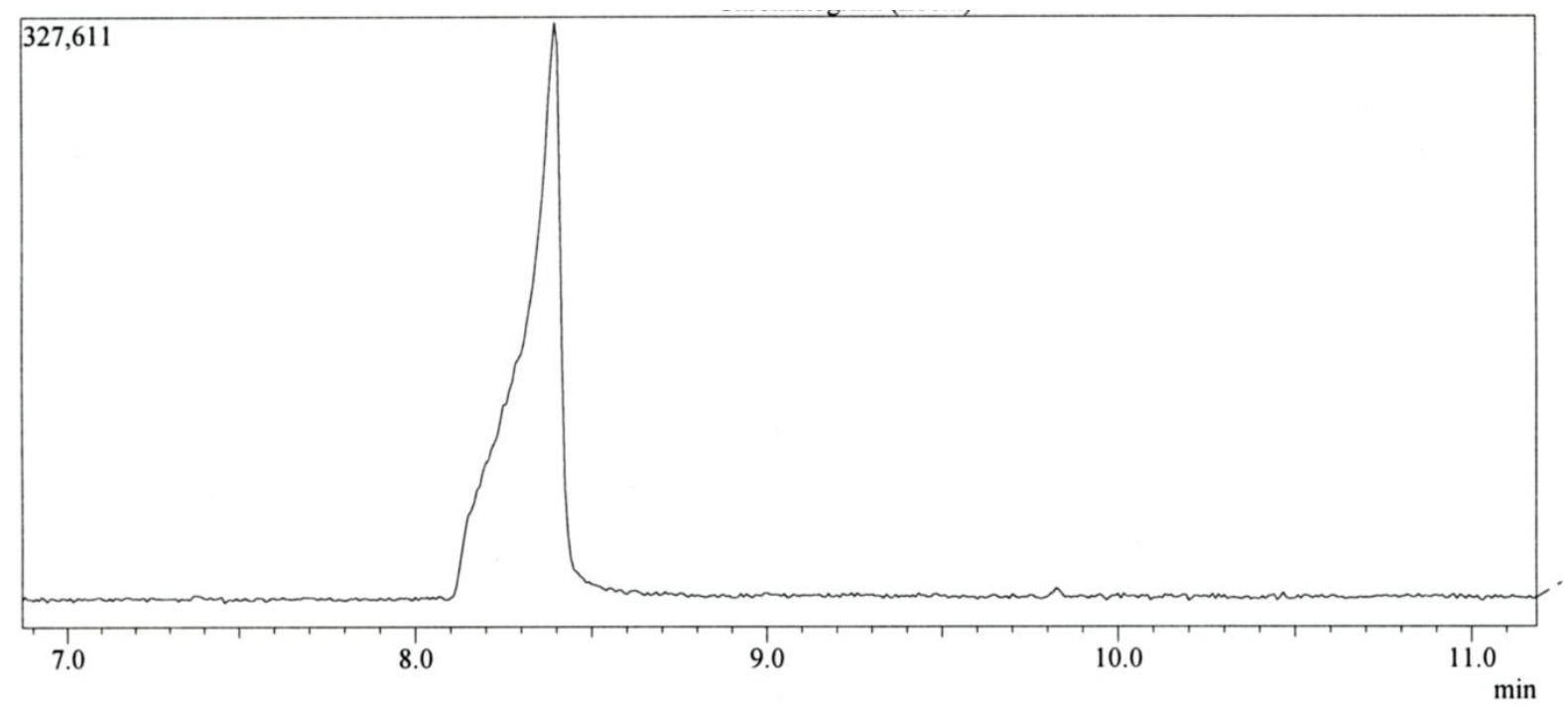

Figure 23. Phentermine peak at $100 \mathrm{ppm}$. Showed signs of peak fronting which is an indication of overloading

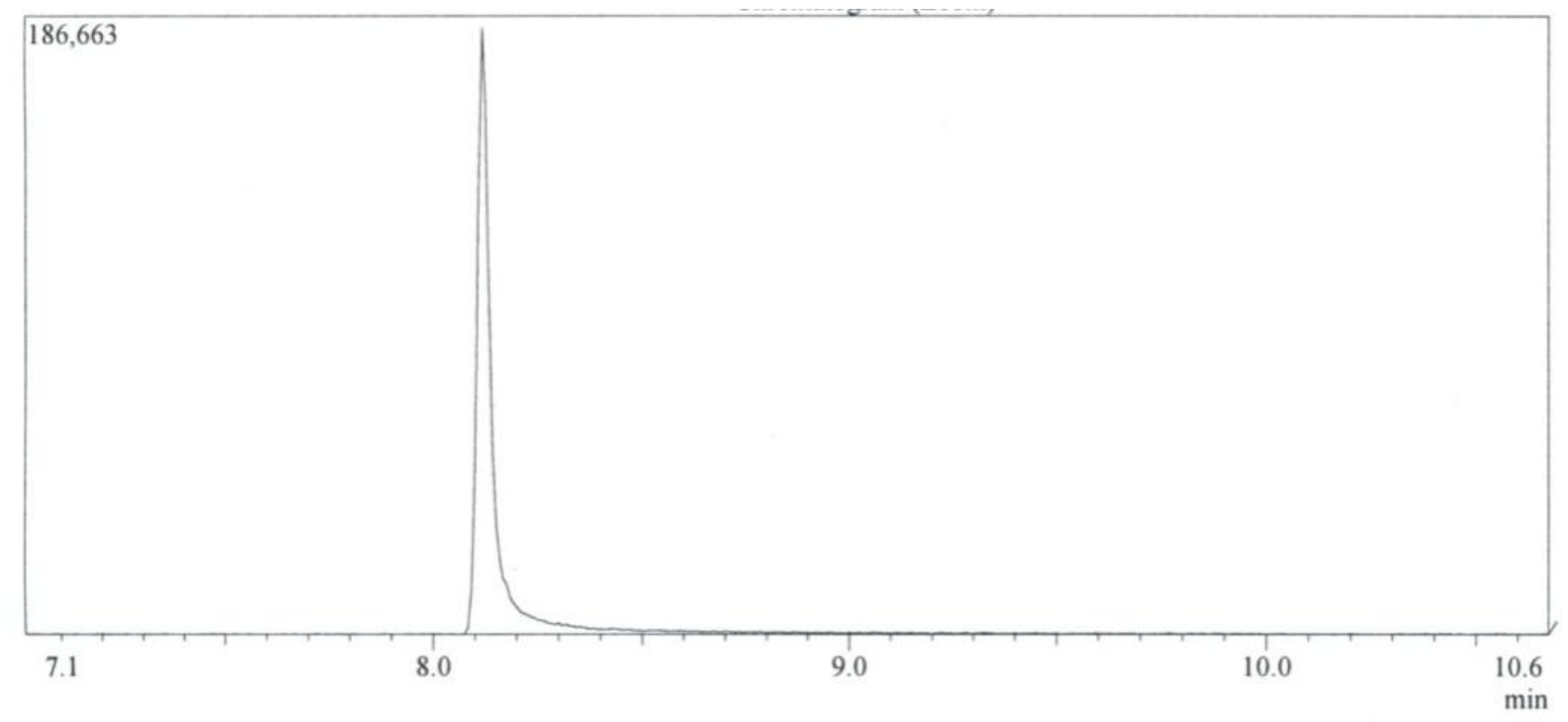

Figure 24. Phentermine peak at 10 ppm. Not showing signs of peak fronting. 
The uncertainty for each drug was calculated to find an acceptable range of LRI.

Methamphetamine and phentermine have higher ranges due to overloading the column (See Table 17). While this problem was easily corrected as discussed below, such overloading could occur during rapid drug screening methods in forensic labs and clearly must be avoided if LRI values are to be used.

Table 17. Uncertainty range for stimulant on the Shimadzu GC

\begin{tabular}{|c|c|c|c|}
\hline & Methamphetamine & D-Amphetamine & Phentermine \\
\hline Average & 1196 & 1141 & 1185 \\
\hline SD & 6 & 2 & 9 \\
\hline Samples Size & 15 & 15 & 15 \\
\hline Uncertainty/Std. Error & 2 & 1 & 14 \\
\hline $\mathrm{df}$ & 14 & 14 & 2.14 \\
\hline t-value & 2.14 & 2.14 & 5 \\
\hline 95\% Conf Dev. & 3 & 1 & 1189 \\
\hline CI $_{\max }$ & 1199 & 1142 & 1180 \\
\hline CI $_{\min }$ & 1193 & 1140 & 0.7 \\
\hline \%RSD & 0.5 & 0.2 & \\
\hline
\end{tabular}


Nine samples of phentermine and three samples of methamphetamine were run again at $10 \mathrm{ppm}$. The new values replaced the values in bold from Table 16. The values are the average of three runs (See Table 18).

Table 18. Modified combined data for methamphetamine and phentermine on the Shimadzu GC. Each Day three replicates were done. Average of the retention indices are shown. **Data for phentermine and methamphetamine were corrected.

\begin{tabular}{|l|c|c|c|c|c|}
\hline Drug Samples & Day 1 & Day 2 & Day 3 & Day 4 & Day 5 \\
\hline Methamphetamine & 1192.90 & 1193.39 & $\mathbf{1 1 9 5 . 5 9}$ & 1193.51 & 1193.34 \\
\hline Phentermine & $\mathbf{1 1 7 7 . 3 0}$ & $\mathbf{1 1 7 7 . 2 3}$ & $\mathbf{1 1 7 7 . 1 7}$ & 1175.12 & 1174.49 \\
\hline
\end{tabular}


The uncertainty for each drug was calculated to find an acceptable range of LRI for methamphetamine and phentermine without overloading the column. The range has decreased and the standard deviation is smaller compared to Table 15 (See Table 19). The uncertainty ranges for barbitals (See Table 20) and depressants (See Table 21) were also found.

Table 19. Modified uncertainty range of LRI for methamphetamine and phentermine on the Shimadzu GC

\begin{tabular}{|c|c|c|}
\hline & Methamphetamine & Phentermine \\
\hline Average & 1194 & 1176 \\
\hline SD & 1 & 1 \\
\hline Samples Size & 15 & 0.3 \\
\hline Uncertainty/Std. Error & 0.3 & 14 \\
\hline $\mathrm{df}$ & 14 & 2.14 \\
\hline t-value & 2.14 & 0.7 \\
\hline 95\% Conf Dev. & 0.5 & 1177 \\
\hline CI $_{\max }$ & 1194 & 1176 \\
\hline CI $_{\min }$ & 1193 & 0.1 \\
\hline \%RSD & 0.1 & \\
\hline
\end{tabular}


Table 20. Uncertainty range for barbitals on the Shimadzu GC

\begin{tabular}{|c|c|c|c|}
\hline & Pentobarbital & Butabarbital & Phenobarbital \\
\hline Average & 1766 & 1688 & 2007 \\
\hline SD & 1 & 1 & 1 \\
\hline Samples Size & 15 & 15 & 15 \\
\hline Uncertainty/Std Error & 0 & 0 & 14 \\
\hline $\mathrm{df}$ & 14 & 14 & 2.14 \\
\hline t-value & 2.14 & 2.14 & 0.3 \\
\hline $95 \%$ Conf Dev. & 0.5 & 0.5 & 2008 \\
\hline CI $_{\max }$ & 1767 & 1688 & 2007 \\
\hline CI $_{\min }$ & 1766 & 1687 & 0.03 \\
\hline$\%$ RSD & 0.05 & 0.06 & \\
\hline
\end{tabular}


Table 21. Uncertainty range for depressants on the Shimadzu GC

\begin{tabular}{|c|c|c|c|}
\hline & Heroin & Oxy & Codeine \\
\hline Average & 2709 & 2626 & 2479 \\
\hline SD & 1 & 1 & 1 \\
\hline Samples Size & 15 & 15 & 0 \\
\hline Uncertainty/Std Error & 0 & 0 & 14 \\
\hline $\mathrm{df}$ & 14 & 14 & 2.14 \\
\hline t-value & 2.14 & 2.14 & 0.6 \\
\hline $95 \%$ Conf Dev. & 0.5 & 0.7 & 2480 \\
\hline CI $_{\max }$ & 2710 & 2627 & 2479 \\
\hline CI $_{\min }$ & 2709 & 2625 & 0.04 \\
\hline$\%$ RSD & 0.03 & 0.05 & \\
\hline
\end{tabular}




\section{Linear retention Index Data- PerkinElmer GC}

The LRI for the PerkinElmer showed little variation. Often, the retention times were identical within each day (See Table 22). The uncertainty values were found for each group of drugs (See Table 23, 24, and 25).

Table 22. Combined linear retention index data on the PerkinElmer GC. Each Day three replicates were done. Average of the retention indices are shown

\begin{tabular}{|c|c|c|c|c|c|}
\hline Drug Samples & Day 1 & Day 2 & Day 3 & Day 4 & Day 5 \\
\hline Methamphetamine & 1190.58 & 1190.48 & 1190.23 & 1190.98 & 1191.46 \\
\hline D-Amphetamine & 1132.58 & 1133.08 & 1132.83 & 1133.08 & 1132.83 \\
\hline Phentermine & 1168.69 & 1169.17 & 1168.42 & 1168.92 & 1168.94 \\
\hline Pentobarbital & 1763.00 & 1762.39 & 1762.39 & 1762.39 & 1762.69 \\
\hline Butabarbital & 1682.46 & 1682.17 & 1681.87 & 1682.46 & 1682.46 \\
\hline Phenobarbital & 1996.31 & 1996.97 & 1995.96 & 1998.32 & 1996.97 \\
\hline Heroin & 2719.70 & 2719.70 & 2720.83 & 2720.83 & 2720.22 \\
\hline Oxycodone & 2610.39 & 2610.39 & 2610.39 & 2611.69 & 2610.09 \\
\hline Codeine & 2455.00 & 2455.56 & 2455.15 & 2455.97 & 2455.56 \\
\hline
\end{tabular}


Table 23.Uncertainty range for stimulants on the PerkinElmer GC

\begin{tabular}{|c|c|c|c|}
\hline & Methamphetamine & D-amphetamine & Phentermine \\
\hline Average & 1191 & 1133 & 1169 \\
\hline SD & 1 & 0 & 0 \\
\hline Samples Size & 15 & 15 & 15 \\
\hline Uncertainty & 0.2 & 0.1 & 0.1 \\
\hline df & 14 & 14 & 14 \\
\hline t-value & 2.14 & 2.14 & 2.14 \\
\hline 95\% Conf Dev. & 0.5 & 0.2 & 0.2 \\
\hline CI (+) & 1191 & 1133 & 1169 \\
\hline CI (-) & 1190 & 1133 & 1169 \\
\hline \%RSD & 0.07 & 0.03 & 0.03 \\
\hline
\end{tabular}


Table 24. Uncertainty range for barbitals on the PerkinElmer GC

\begin{tabular}{|c|c|c|c|}
\hline & Pentobarbital & Butabarbital & Phenobarbital \\
\hline Average & 1763 & 1682 & 1997 \\
\hline SD & 0 & 0 & 1 \\
\hline Samples Size & 15 & 15 & 15 \\
\hline Uncertainty & 0.1 & 0.1 & 0.2 \\
\hline df & 14 & 14 & 14 \\
\hline t-value & 2.14 & 2.14 & 2.14 \\
\hline $95 \%$ Conf Dev. & 0.2 & 0.2 & 0.5 \\
\hline CI (+) & 1763 & 1682 & 1998 \\
\hline CI (-) & 1762 & 1682 & 1997 \\
\hline$\%$ RSD & 0.02 & 0.02 & 0.04 \\
\hline
\end{tabular}


Table 25. Uncertainty range for depressants on the PerkinElmer GC

\begin{tabular}{|c|c|c|c|}
\hline & Heroin & Oxycodone & Codeine \\
\hline Average & 2720 & 2611 & 2455 \\
\hline SD & 1 & 1 & 1 \\
\hline Samples Size & 15 & 15 & 15 \\
\hline Uncertainty & 0.2 & 0.2 & 0.1 \\
\hline df & 14 & 14 & 14 \\
\hline t-value & 2.14 & 2.14 & 2.14 \\
\hline $95 \%$ Conf Dev. & 0.4 & 0.4 & 0.3 \\
\hline CI (+) & 2721 & 2611 & 2456 \\
\hline CI (-) & 2720 & 2610 & 2455 \\
\hline$\%$ RSD & 0.03 & 0.02 & 0.02 \\
\hline
\end{tabular}


The LRI for each drug group was calculated and averaged for three replicates from the average ladder from the modified temperature program from Method 2 (Table 26,27, and 28). The LRI of the 9 drugs found were all within the uncertainty range found for each drug using the parameters from Method 1 (or within 1 riu) suggesting that the differences between the two GC/MS methods do not influence LRI of the nine drugs selected. 
Table 26. LRI data for stimulants on the PerkinElmer GC using Method 3 parameters

\begin{tabular}{|c|c|c|c|}
\hline & Methamphetamine & D-Amphetamine & Phentermine \\
\hline Run 1 & 1189 & 1132 & 1168 \\
\hline Run 2 & 1189 & 1132 & 1168 \\
\hline Run 3 & 1190 & 1132 & 1168 \\
\hline & & & \\
\hline Average & 1189 & 1132 & 1168 \\
\hline SD & 0.8 & 0.0 & 0.4 \\
\hline \%RSD & 0.06 & 0.0 & 0.04 \\
\hline
\end{tabular}


Table 27. LRI data for barbitals on the PerkinElmer GC using Method 3 parameters

\begin{tabular}{|c|c|c|c|}
\hline & Pentobarbital & Butabarbital & Phenobarbital \\
\hline Run 1 & 17623 & 1681 & 1997 \\
\hline Run 2 & 1763 & 1681 & 1997 \\
\hline Run 3 & 1763 & 1682 & 1997 \\
\hline & & & 1997 \\
\hline Average & 1763 & 1681 & 0.00 \\
\hline SD & 0.00 & 0.5 & 0.00 \\
\hline \%RSD & 0.00 & 0.03 & \\
\hline
\end{tabular}


Table 28. LRI data for depressants on the PerkinElmer using Method 3 parameters

\begin{tabular}{|c|c|c|c|}
\hline & Heroin & Oxycodone & Codeine \\
\hline Run 1 & 2720 & 2610 & 2456 \\
\hline Run 2 & 2720 & 2612 & 2454 \\
\hline Run 3 & 2720 & 2612 & 2456 \\
\hline & & & \\
\hline Average & 2720 & 2611 & 2455 \\
\hline SD & 0.00 & 0.7 & 0.7 \\
\hline \%RSD & 0.00 & 0.03 & 0.03 \\
\hline
\end{tabular}




\section{Combined LRI data from the Shimadzu GC and the PerkinElmer GC}

A one-way ANOVA was calculated for each drug from both instruments. Every drug had an $\mathrm{F}$ value higher than the $\mathrm{F}_{\text {critical }}$ value and a p-value less than 0.05 , which rejects the null hypothesis that the means are the same. Based on a one-way ANOVA, the LRI data cannot be combined for the both instruments. An example of the one-way ANOVA table is shown below for pentobarbital (See Table 29).

Table 29. One-way ANOVA table for pentobarbital from both instruments

\begin{tabular}{|c|c|c|c|c|c|c|}
\hline $\begin{array}{c}\text { Source of } \\
\text { Variation }\end{array}$ & $S S$ & $d f$ & $M S$ & $F$ & P-value & F crit \\
\hline Between Groups & 91.47 & 1 & 91.47 & $\mathbf{1 9 5 . 2 8}$ & $\mathbf{3 . 7 8 \times \mathbf { 1 0 }} \mathbf{1 4}^{\mathbf{4}}$ & $\mathbf{4 . 2 0}$ \\
\hline Within Groups & 13.11 & 28 & 0.47 & & & \\
\hline & & & & & & \\
\hline Total & 104.59 & 29 & & & & \\
\hline
\end{tabular}




\section{Resolution study for methamphetamine and phentermine}

Methamphetamine and Phentermine were equally combined and run through both the Shimadzu GC and PerkinElmer GC at concentrations of $10 \mathrm{ppm}$ and $100 \mathrm{ppm}$. The temperature ramp programs were the same (Method 1 and Method 3). The resolution of peaks was calculated to determine if the peaks were fully separated. The PerkinElmer at $10 \mathrm{ppm}$ and $100 \mathrm{ppm}$ (See Figure 27 and 28) and 10 ppm on Shimadzu (See Figure 25) were fully resolved while the 100ppm on the Shimadzu was not (See Figure 26).

The linear retention indices were calculated for the $100 \mathrm{ppm}$ methamphetamine and amphetamine mixture for the Shimadzu GC (See Table 30). The LRI do show a difference between the two drugs where the resolution of the peaks did not.

Table 30. LRI of 10 ppm mixture of methamphetamine and phentermine on the Shimadzu GC

\begin{tabular}{|c|c|c|}
\hline & Methamphetamine & Phentermine \\
\hline Run 1 & 1200.75 & 1181.39 \\
\hline Run 2 & 1201.84 & 1183.71 \\
\hline Run 3 & 1201.91 & 1184.25 \\
\hline Average & 1201.50 & 1183.12 \\
\hline SD & 0.65 & 1.52 \\
\hline \%RSD & 0.05 & 0.13 \\
\hline
\end{tabular}




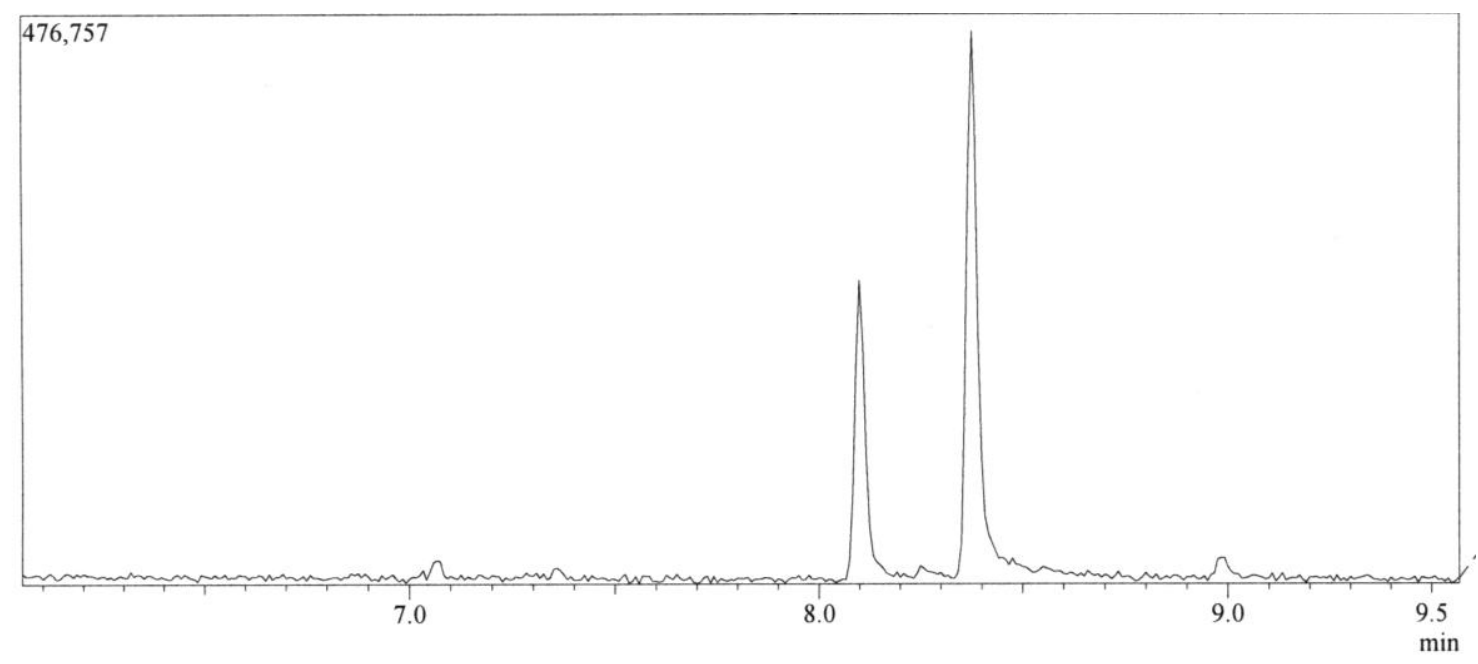

Figure 25. Methamphetamine and phentermine at 10 ppm on the Shimadzu GC

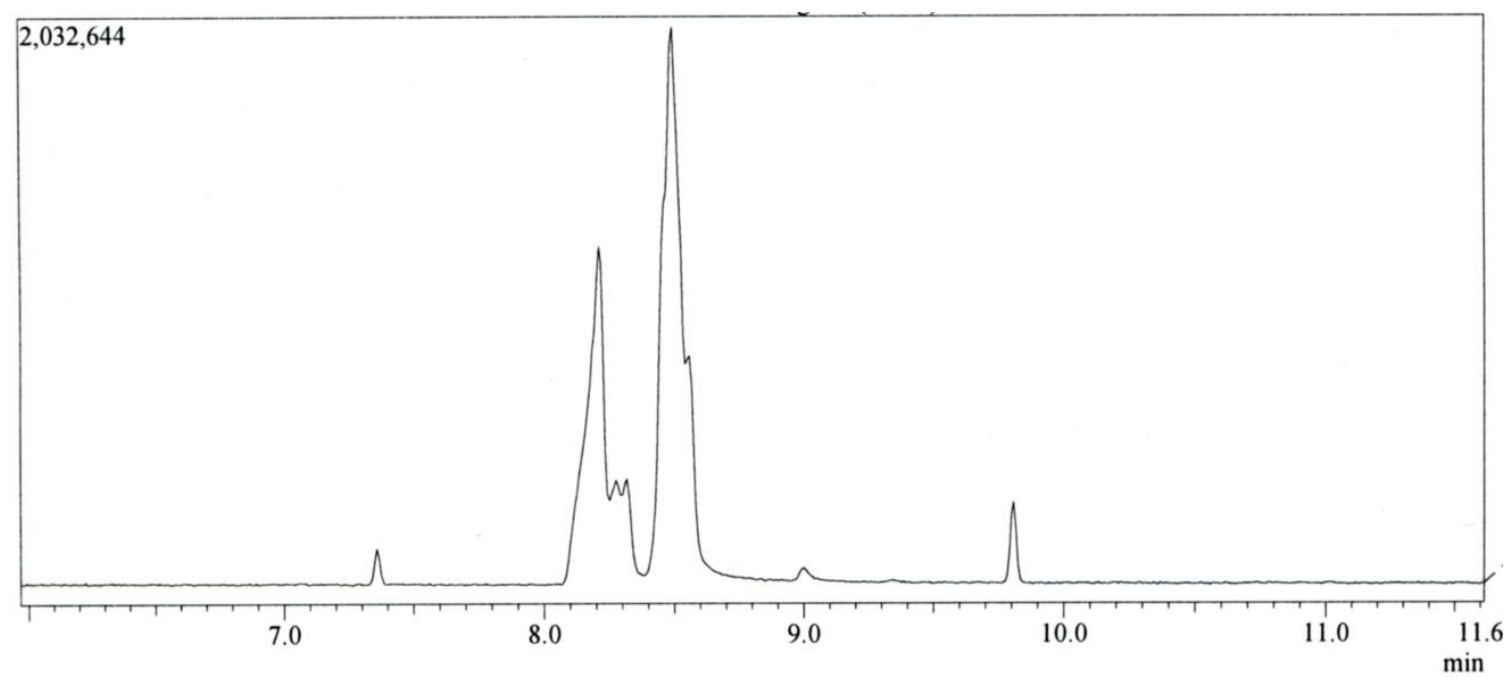

Figure 26. Methamphetamine and phentermine at 100 ppm on the Shimadzu GC 


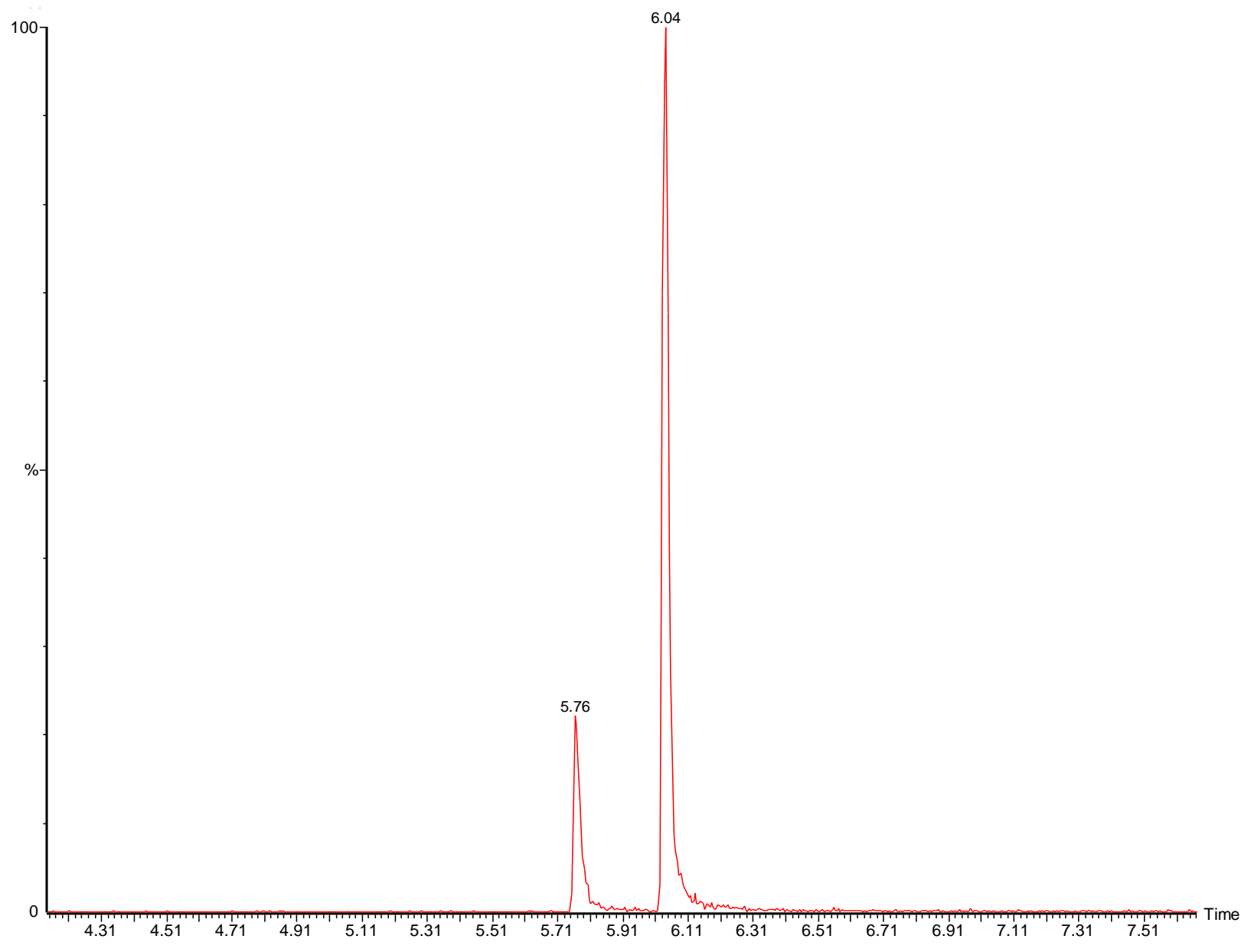

Figure 27. Methamphetamine and phentermine at 10 ppm on the PerkinElmer GC 


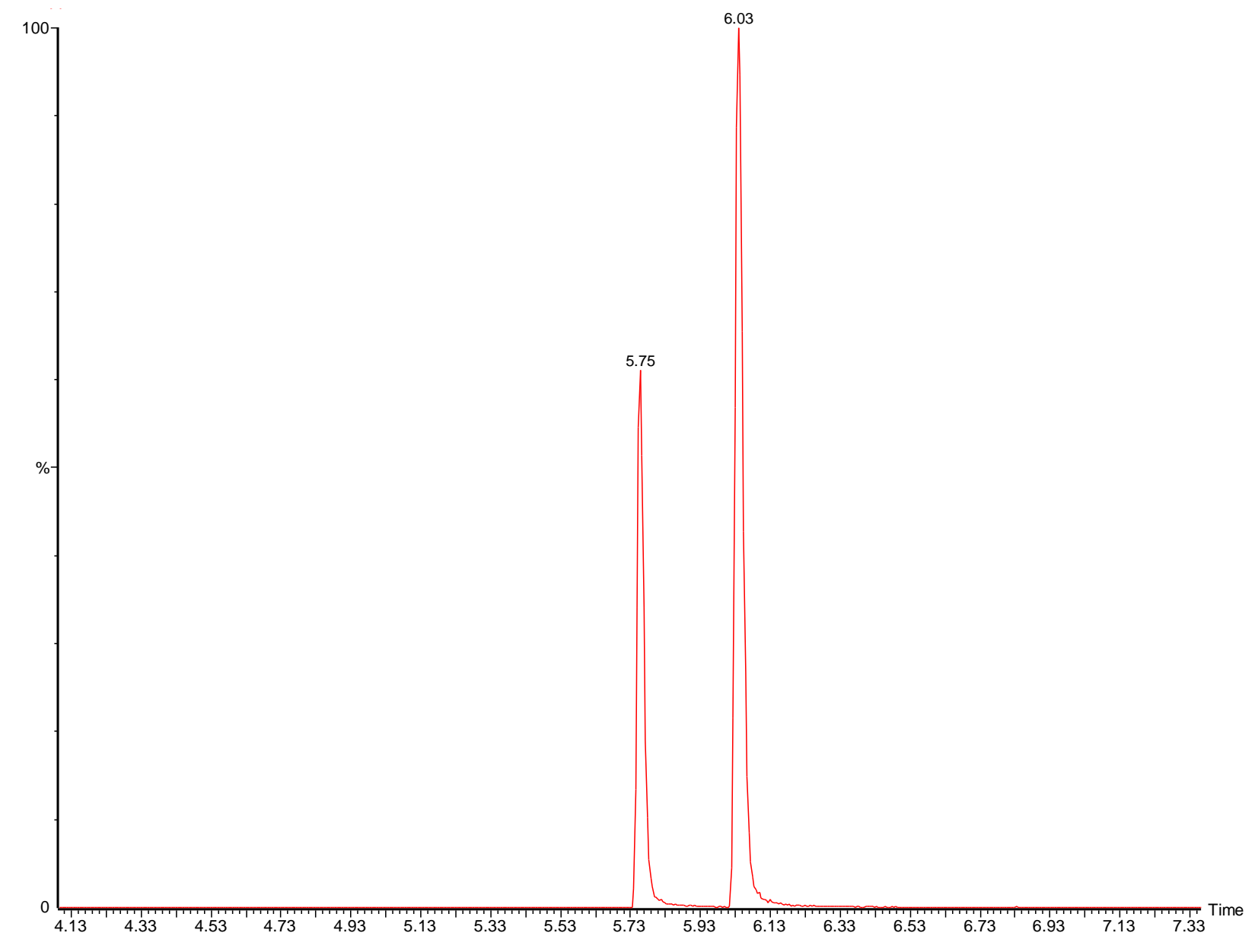

Figure 28. Methamphetamine and phentermine at 100 ppm on the PerkinElmer GC 


\section{Conclusion}

When the hydrocarbon ladder was run on the Shimadzu GC, the alkane ladder spanned C7-C29 and eventually C8-C29 after an adjustment to the start time on the MS to incorporate a solvent delay. The PerkinElmer GC spanned C10-C33. The temperature ramp programs were the same with only a difference in the injector port temperature $\left(30^{\circ} \mathrm{C}\right.$ lower in PerkinElmer GC) and the interface temperature $\left(10^{\circ} \mathrm{C}\right)$. A second experiment using the same experimental conditions were performed on the PerkinElmer and the same linear alkanes were detected, C10C33. This indicates that the injector port temperature and interface temperature is not influencing the amount of alkanes seen in each GC.

One explanation for more alkanes seen later in the PerkinElmer GC is due to the thinner film thickness of the column. It is possible that the alkanes are not retained as much as they are in the thicker film of the column in the Shimadzu GC. The earlier alkanes not shown are believed to elute during the solvent delay of 3 minutes. Since there is less interaction with the thinner film, it is possible that more of the beginning alkanes $(\mathrm{C} 7, \mathrm{C} 8$, and $\mathrm{C} 10)$ are not seen in the PerkinElmer GC compared to the thicker film in the Shimadzu GC.

The hydrocarbon ladders were run for approximately a month. The hydrocarbon ladders for the Shimadzu GC and the PerkinElmer GC did show a difference in the variances of the means, therefore a hydrocarbon ladder should be run at least once before running any drug samples for the same day.

The film thickness of the Restek ${ }^{\circledR}$ column in the Shimadzu GC is double that of the Phenomenex ${ }^{\circledR}$ column in the PerkinElmer GC. Methamphetamine and phentermine had to be diluted more to $10 \mathrm{ppm}$ in order to have a more Gaussian shape. When the LRI was calculated 
between these two drugs, the LRI was very nearly identical in some cases at $100 \mathrm{ppm}$. For the first two sets of data, methamphetamine was run at $10 \mathrm{ppm}$ while phentermine was run at 100 ppm. New samples were made up to $100 \mathrm{ppm}$ so the third set of data had both drugs run at 100 ppm for the third day of sampling. While the LRI is expected to be closer, the two drugs should have a larger difference that what was being calculated. Both compounds at 100 ppm exhibited peak fronting in the first three sets of data for the Shimadzu GC. The concentration was lowered to $10 \mathrm{ppm}$ for each compound for the fourth and fifth sets of data to avoid peak fronting. After the adjustment of phentermine to $10 \mathrm{ppm}$, the peak was no longer showing peak fronting. There was not any peak fronting for these two compounds with the Phenomenex ${ }^{\circledR}$ column in the PerkinElmer GC at 100ppm so the concentration remained the same for the five runs on that instrument.

When the phentermine and methamphetamine were run at $10 \mathrm{ppm}$, no peak fronting was seen. When this correction is not made, the LRI values are too similar to be differentiated. Care should be taken in indicating peak fronting is not shown in any of the peaks since it does influence the calculation of LRI. The variability was much lower when these two drugs were run at a lower concentration. There were also about 20 riu apart which is to be expected from previous literature.

The PerkinElmer GC showed a smaller variation of the retention times of the nine drugs samples. Often the retention times were identical between data sets. This indicates that a thinner film thickness for a DB-5 equivalent stationary phase is ideal. The equilibrium time was double that of the Shimadzu GC, meaning that it had more time to equilibrate the temperature of the column. This also could account for the small variability of retention times. When the mixture of methamphetamine and phentermine was examined, sufficient resolution was still obtained. 
However, the mass spectrum and library search produce similar results. This could indicate a false positive when using a mass spectrometer to identify methamphetamine and phentermine. Even when mixed together, retention indices were able to differentiate the drugs when the peaks were not fully resolved.

The modified temperature program used from Method 3 for the PerkinElmer GC suggested that the LRI values could be combined with the LRI data using Method 2 for the PerkinElmer GC. Even though some alkanes were significantly different (t-test, 95\% confidence) from the hydrocarbon ladder with 15 replicates, it did not influence LRI because those alkanes were not used for the calculation of LRI. However, this test only applies when the variance is measurable. More replication using the parameters from Method 3 would need to be completed before stating that the two data sets could be safely combined.

Using a paired t-test, the hydrocarbon ladders from Method 2 and Method 3 were not significantly different. The alkanes C29-C33 were significantly different but these alkanes spend more time in the column and have a slightly larger variability. If these alkanes are used to find RI, the conditions should be exactly the same for comparison. It is suggested that the change in interface and injection port temperature did not influence that two data sets on the PerkinElmer GC since the LRI calculated using the modified temperature program did fall within the uncertainty range for each drug (or within 1 riu). Regardless, the LRI values between the two instruments (Shimadzu GC and PerkinElmer GC) cannot be combined.

Uncertainty values were generated for each drug on each GC. This could increase the value of LRI when the same column and experimental parameters are used in other laboratories. Film thickness of the same stationary phase is known to affect LRI. It was shown through this 
study that the data cannot be combined. However, some of the uncertainty ranges are within 10 riu (the drugs that eluted before 12 minutes) while others are over 40 riu apart. LRI is reported in the NIST database. However, the values are often estimated and much higher than the actual values. In order for LRI to be used and compared, the same experimental parameters must be used. Each peak needs to be examined for peak fronting or the LRI value will not be accurate.

For a universal database to be used (i.e., one that could be used amongst and between laboratories), standardization on the two most common columns used in drug analysis (DB-1 and DB-5 equivalents) must be performed. If the same experimental conditions are used, the data should be comparable. An amine column may be better suitable for drug analysis for LRI since most drugs are basic. Overall, LRI can separate samples that co-elute which can prevent false positives as well as false negatives. Also, since this method is rapid and simple, it can aid in backlog which would benefit the forensic community, specifically drug analysis, immensely. 


\section{References}

Babushok, V. I., Linstrom, P. J., Reed, J. J., Zenkevich, I. G., Brown, R. L., Mallard, W. G., et al. (2007). Development of a database of gas chromatographic retention properties of organic compounds. Journal of Chromatography a, 1157(1-2), 414-421. doi:10.1016/j.chroma.2007.05.044

Christ, D. W., Noomano, P., Rosas, M., \& Rhone, D. (1988). Retention indexes by wide-bore capillary gas-chromatography with nitrogen-phosphorus detection. Journal of Analytical Toxicology, 12(2), 84-88.

Clarke's Analysis of Drugs and Poisons. (2004). Chicago: Pharmaceutical Press.

Lebron-Aguilar, R., Quintanilla-Lopez, J. E., \& Garcia-Dominguez, J. A. (2002). Improving the accuracy of kovats' retention indices in isothermal gas chromatography. Journal of Chromatography A, 945(1-2), 185-194. doi:10.1016/S0021-9673(01)01463-7

Levin, B. (2010). Principles of Forensic Toxicology, $3^{\text {rd }}$ ed. Washington D.C.: American Association for Clinical Chemistry.

Linstromm P.J and Mallard,W.G, Eds., NIST Chemistry WebBook, NIST Standard Reference Database Number 69, National Institute of Standards and Technology, Gaithersburg MD, 20899, http://webbook.nist.gov, (retrieved March 31, 2013).

Locos, O., \& Reynolds, D. (2012). The characterization of 3,4-dimethylmethcathinone (3,4DMMC). Journal of Forensic Sciences, 57(5), 1303-1306. doi:10.1111/j.15564029.2012.02142.x

Mead, J. SWGDRUG meeting minutes: Scientific Working Group for the Analysis of Seized Drugs, January 10-12 (2012)

National Academy of Sciences.( 2009). Strengthening Forensic Sciences in the United States. Washington D.C.: The National Academies Press. Available from: http://www.nap.edu/catalog.php?record_id=12589

Oyama, N., Sano, T., Syoyama, M., \& Maeda, K. (1987). Studies on systematic analysis of poisonous compounds in forensic chemistry 2. Application of capillary column gaschromatography to substance identification by means of retention indexes. Eisei KagakuJapanese Journal of Toxicology and Environmental Health, 33(5), 342-348.

Placke, A. SWGDRUG meeting minutes: Scientific Working Group for the Analysis of Seized Drugs, July 5-7 (2011).

Scientific Working Group for the Analysis of Seized Drugs (SWGDRUG). 2013. Recommendations. Retrieved March 1, 2013 from http://www.swgdrug.org. 
Stein, S. E., Babushok, V. I., Brown, R. L., \& Linstrom, P. J. (2007). Estimation of Kovats retention indices using group contributions. Journal of Chemical Information and Modeling, 47(3), 975-980. doi:10.1021/ci600548y

Storm, K.J, McDonald Smiley, H, Stout, P.R, Ropero-Miller, J.D., Bachrach, J. (2010). NIJ Controlled Substances Case Processing Study. Document No. 233830. RTI International.

Toth, A., \& Praszna, L. (1998). Improvement of the reproducibility of retention indices in temperature-programmed gas-liquid chromatography. Part I. DB-1 column. Flavour and Fragrance Journal, 13(3), 196-202. doi:10.1002/(SICI)1099-1026(199805/06)13:3

U.S. Department of Justice, Drug Enforcement Administration, Office of Diversion Control. (2011). National Forensic Laboratory Information System: Year 2008 Annual Report. Washington, DC: Author

U.S. Department of Justice, Federal Bureau of Investigation. (2011). Crime in the United States, 2011. Retrieved March 25, 2013, from http://www.fbi.gov/about-us/cjis/ucr/crime-in-the-u.s/2011/crime-in-the-u.s.2011/persons-arrested/persons-arrested

United States Drug Enforcement Administration. (2012). Drug Fact Sheet. Retrieved March 25, 2013, from

http://www.justice.gov/dea/druginfo/factsheets.shtml

Zellner, B. d., Bicchi, C., Dugo, P., Rubiolo, P., Dugo, G., \& Mondello, L. (2008). Linear retention indices in gas chromatographic analysis: A review. Flavour and Fragrance Journal, 23(5), 297-314. doi:10.1002/ffj.1887

Zhang, J., Fang, A., Wang, B., Kim, S. H., Bogdanov, B., Zhou, Z., et al. (2011). iMatch: A retention index tool for analysis of gas chromatography-mass spectrometry data. Journal of Chromatography A, 1218(37), 6522-6530. doi:10.1016/j.chroma.2011.07.039 


\section{Appendix A: Shimadzu GC Data}

\section{Methamphetamine}
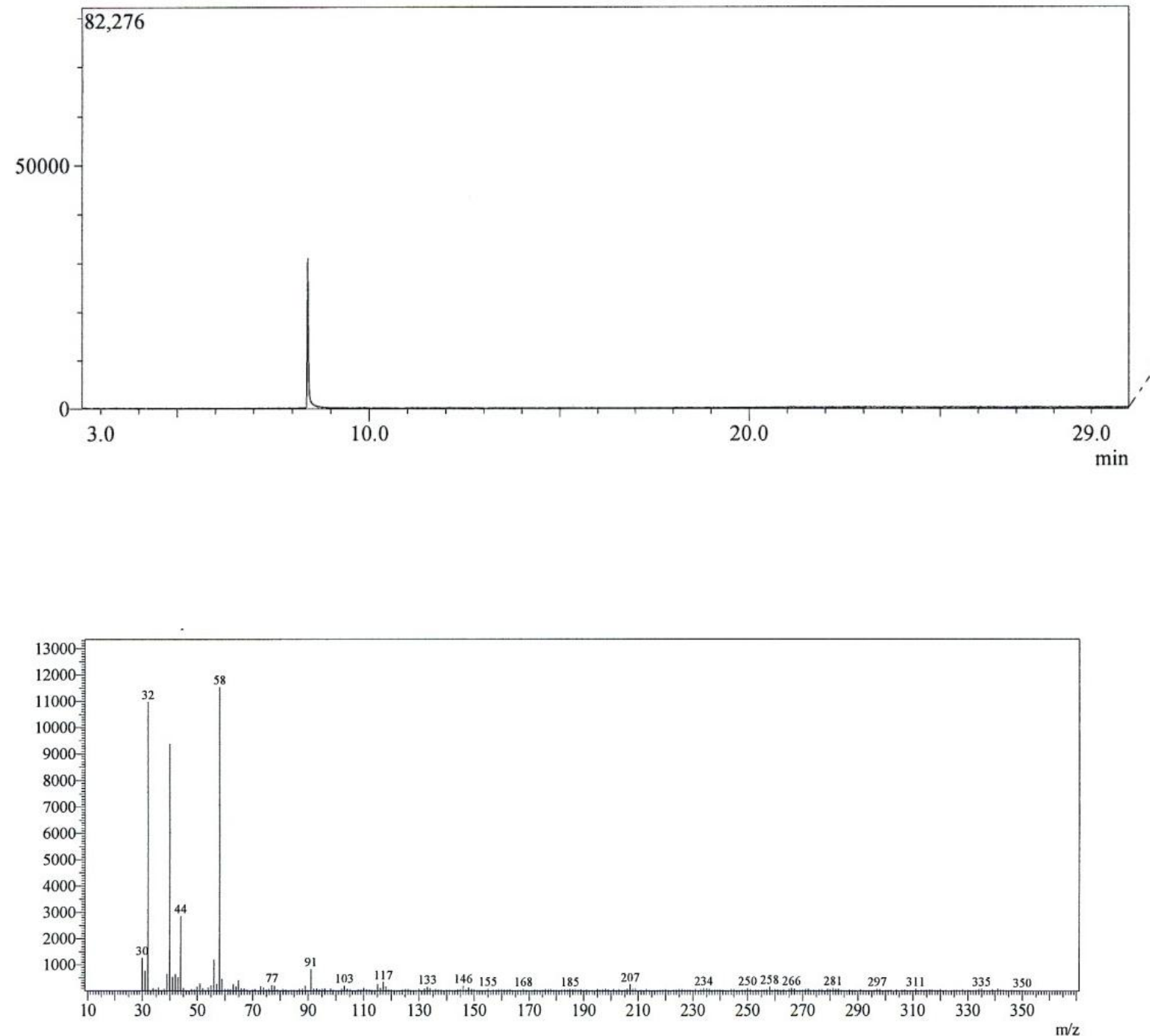


\section{Amphetamine}
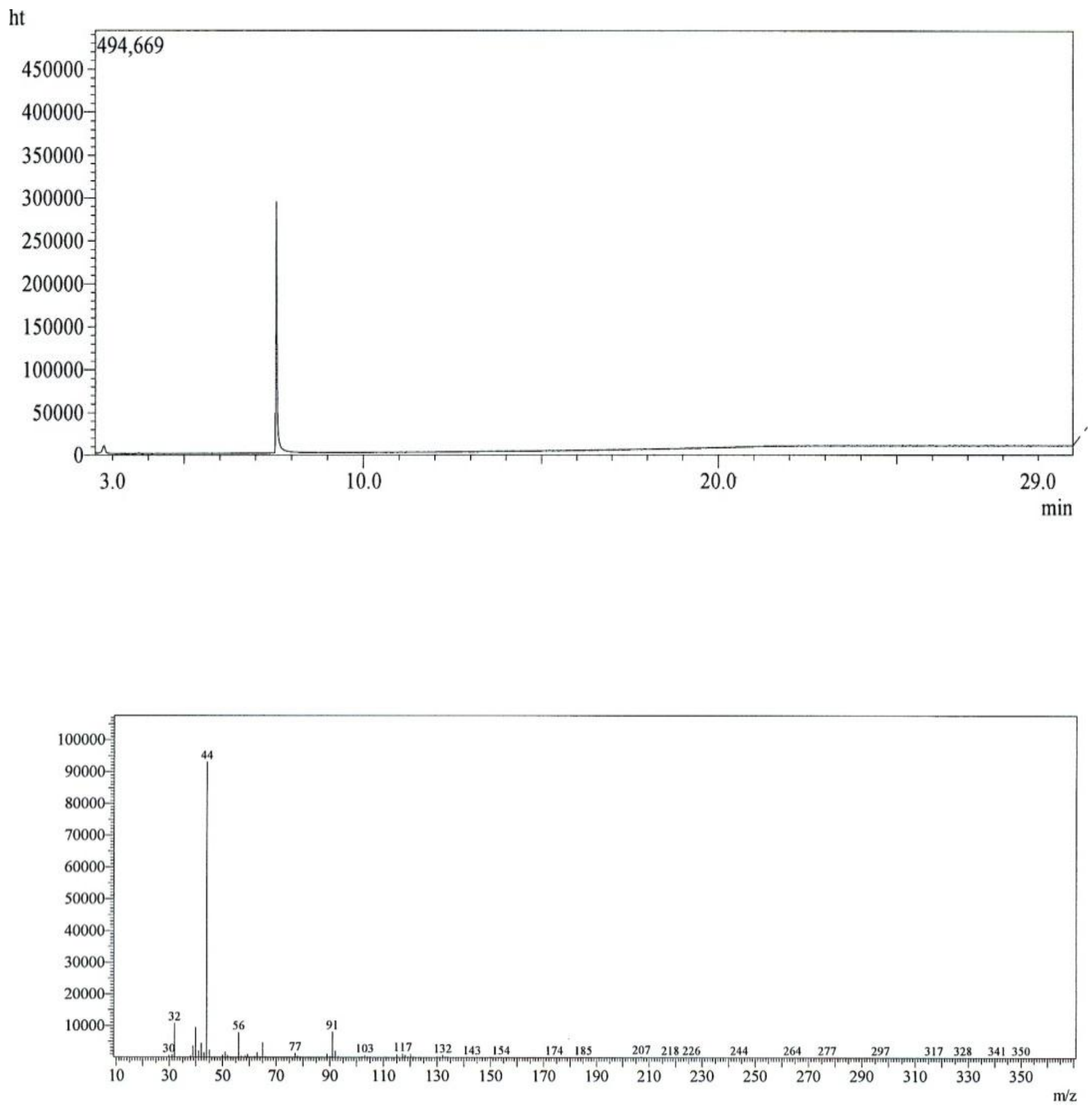


\section{Phentermine}
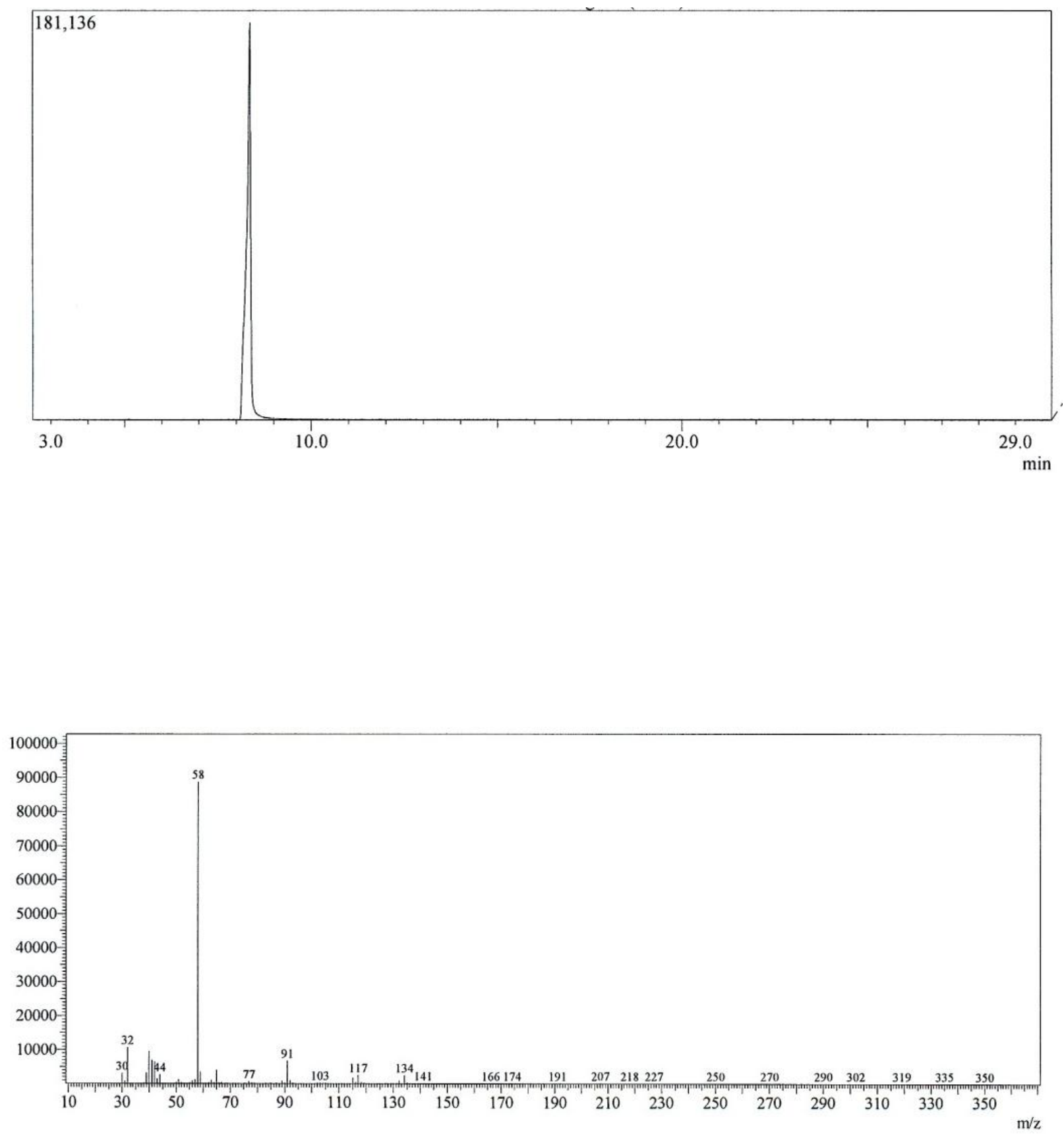


\section{Heroin}

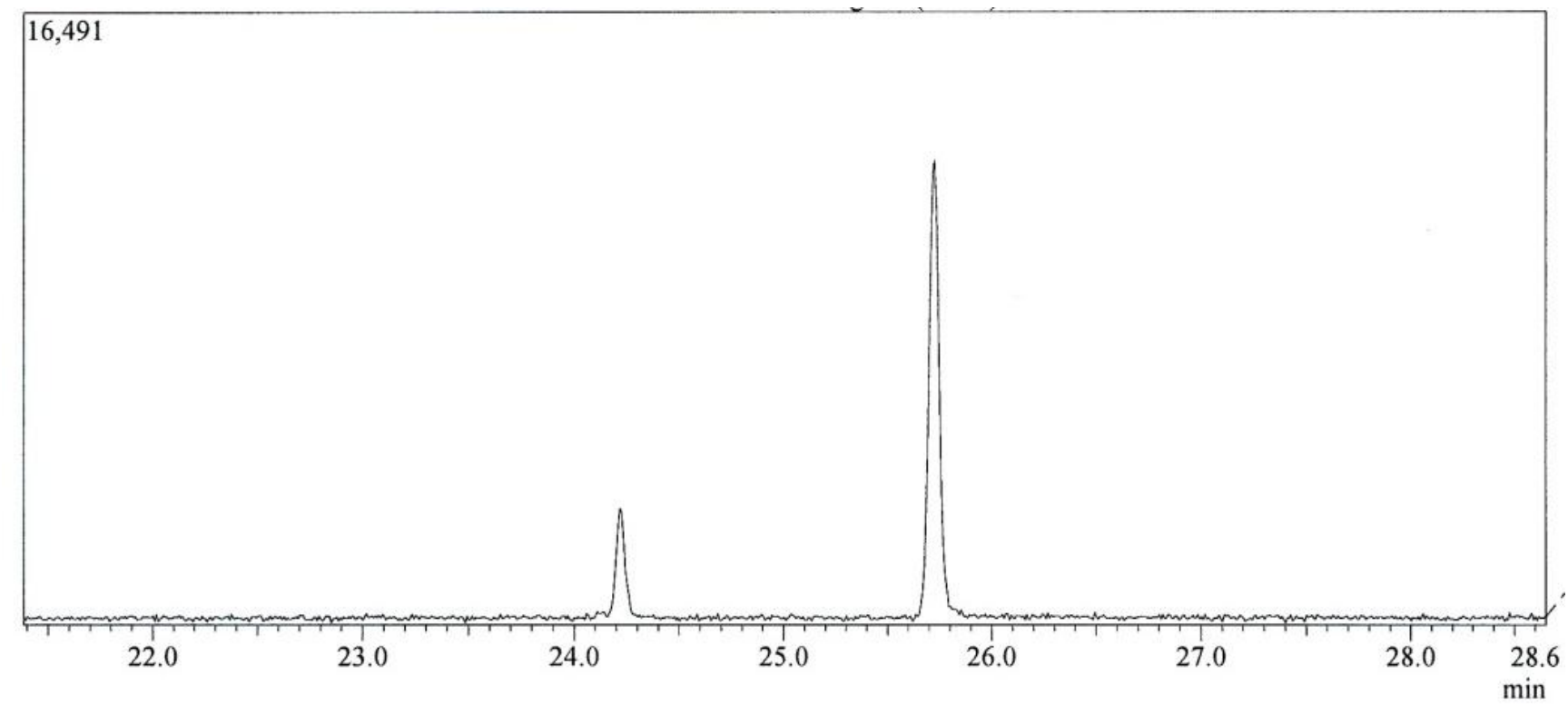

**Two peaks of heroin were seen on both instruments. This is due to degradation of the standard. The second larger peak was used for the calculation of RI

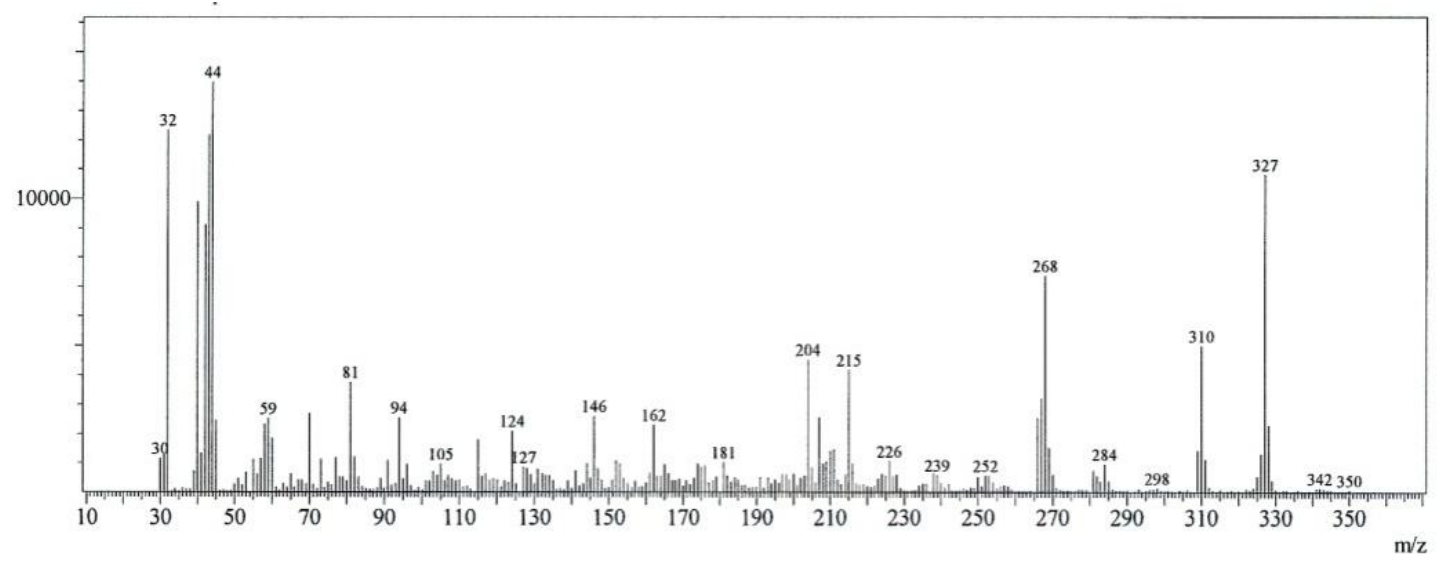




\section{Oxycodone}
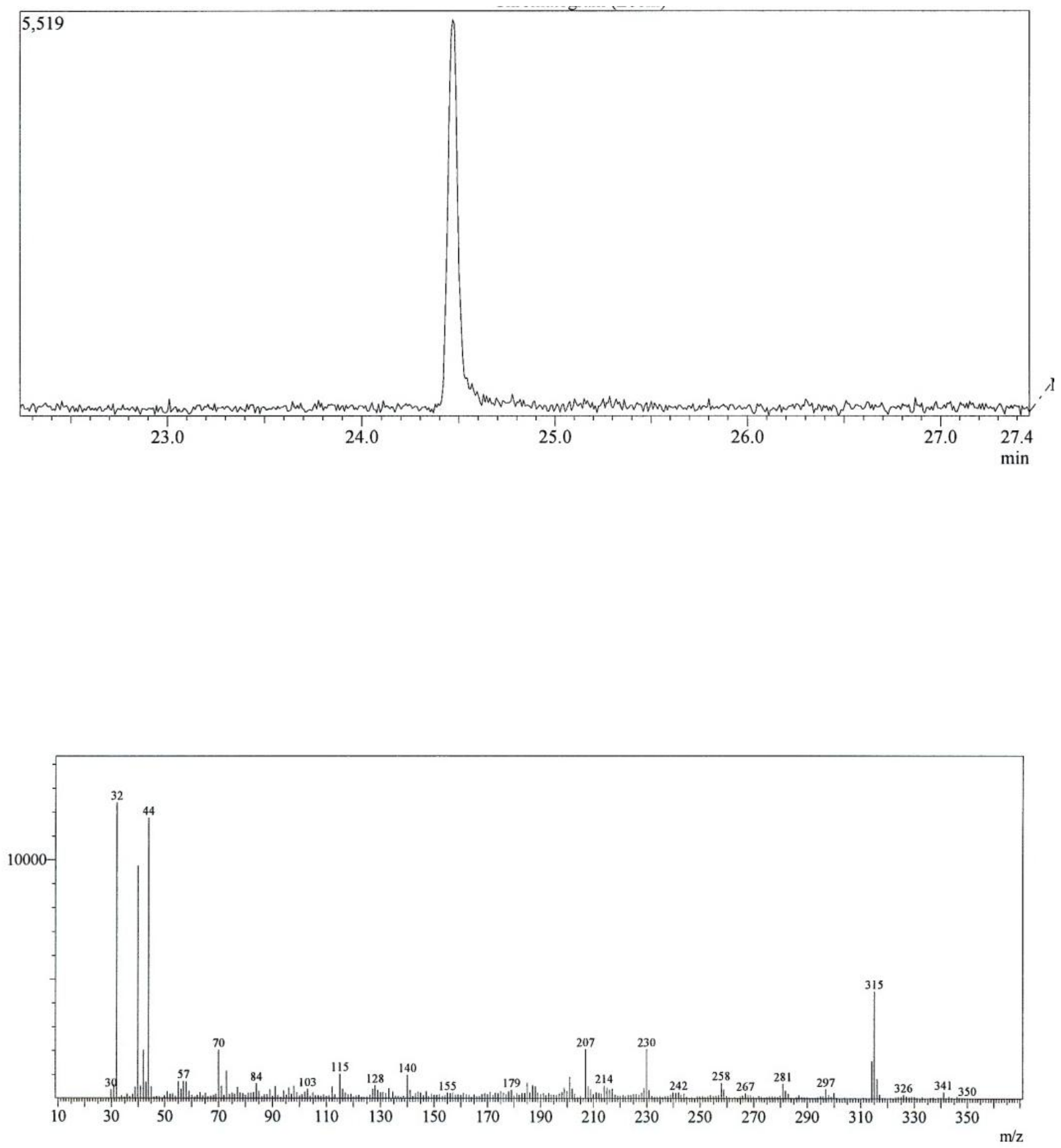


\section{Codeine}
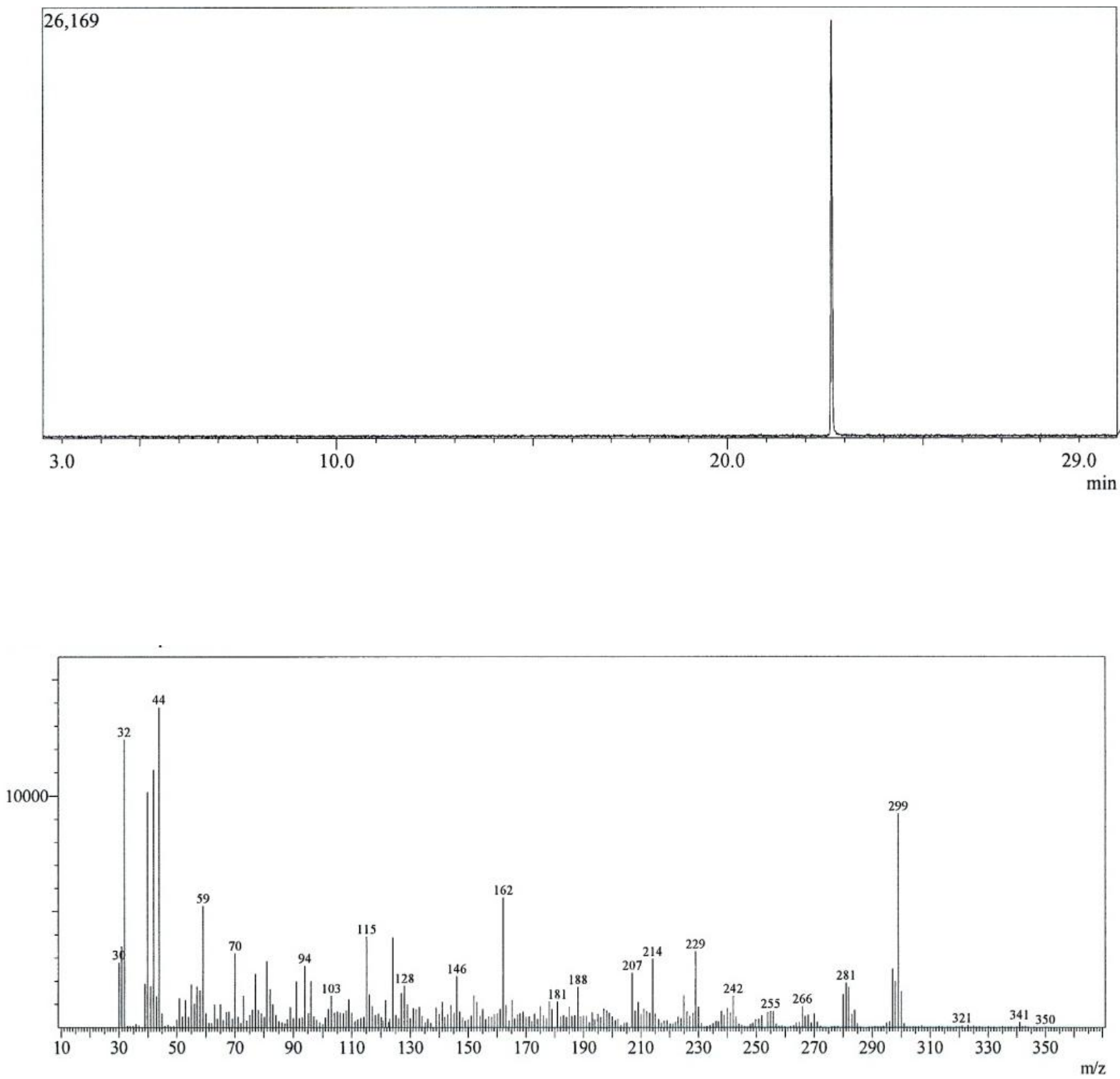


\section{Pentobarbital}
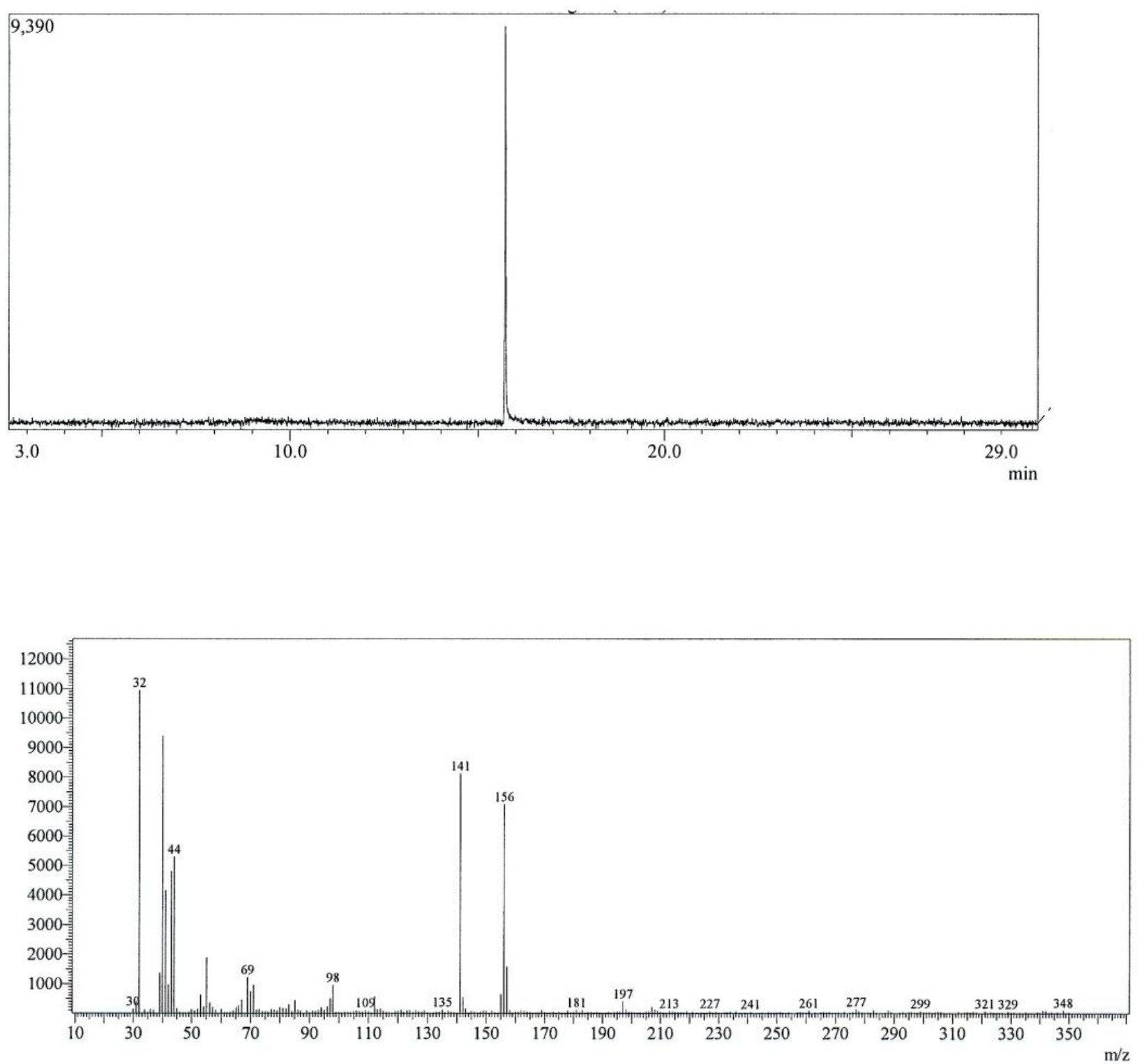


\section{Butabarbital}

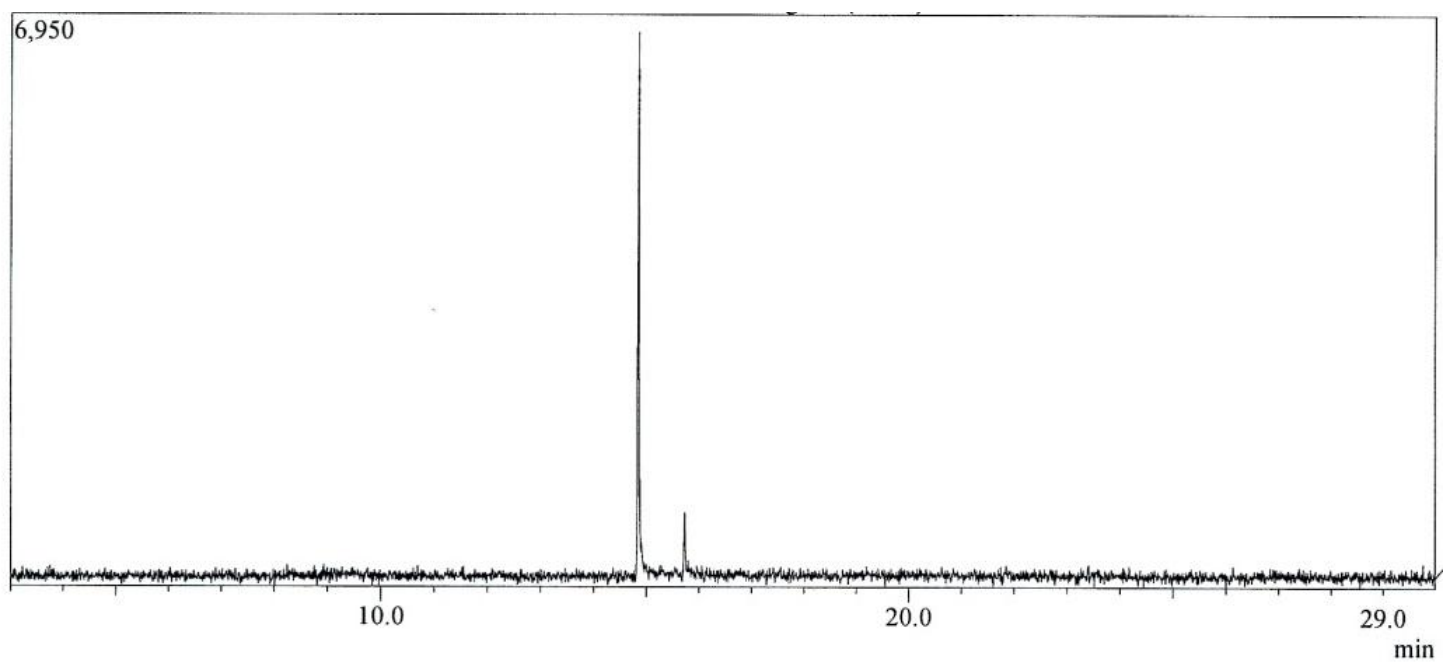

Line\#:1 R.Time: 14.850 (Scan\#:1423)

MassPeaks:302

RawMode:Single 14.850(1423) BasePeak:39.90(9806)

BG Mode:None Group 1 - Event 1

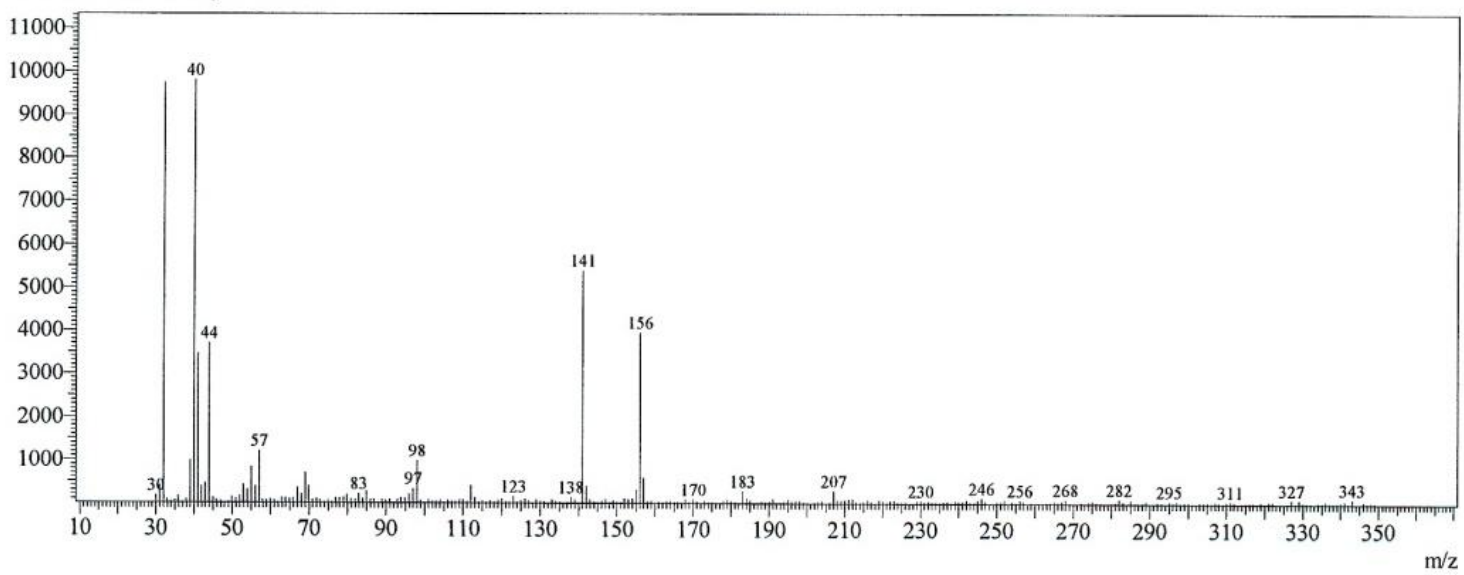




\section{Phenobarbital}
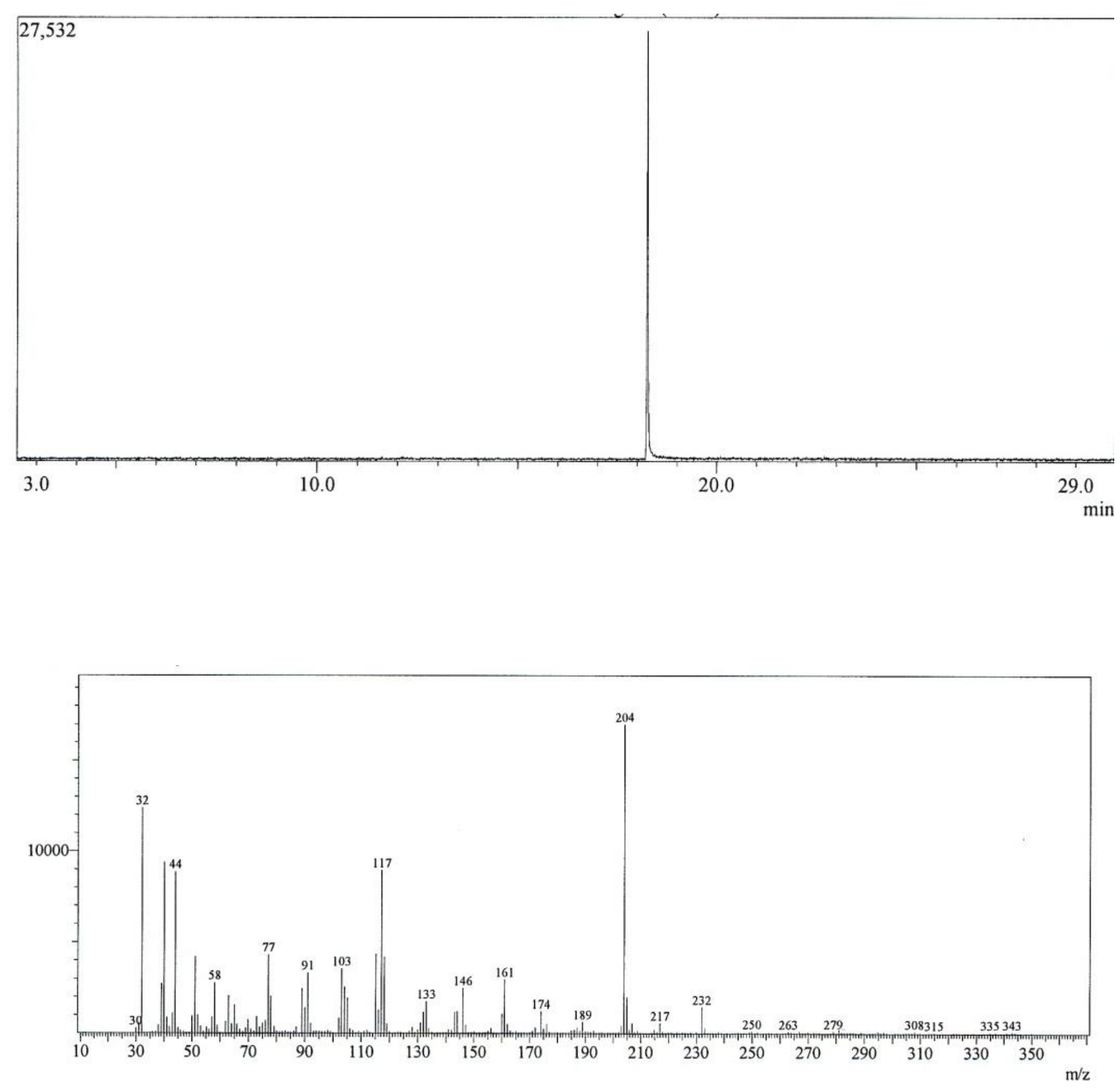


\section{Appendix B: PerkinElmer GC Data}

Methamphetamine
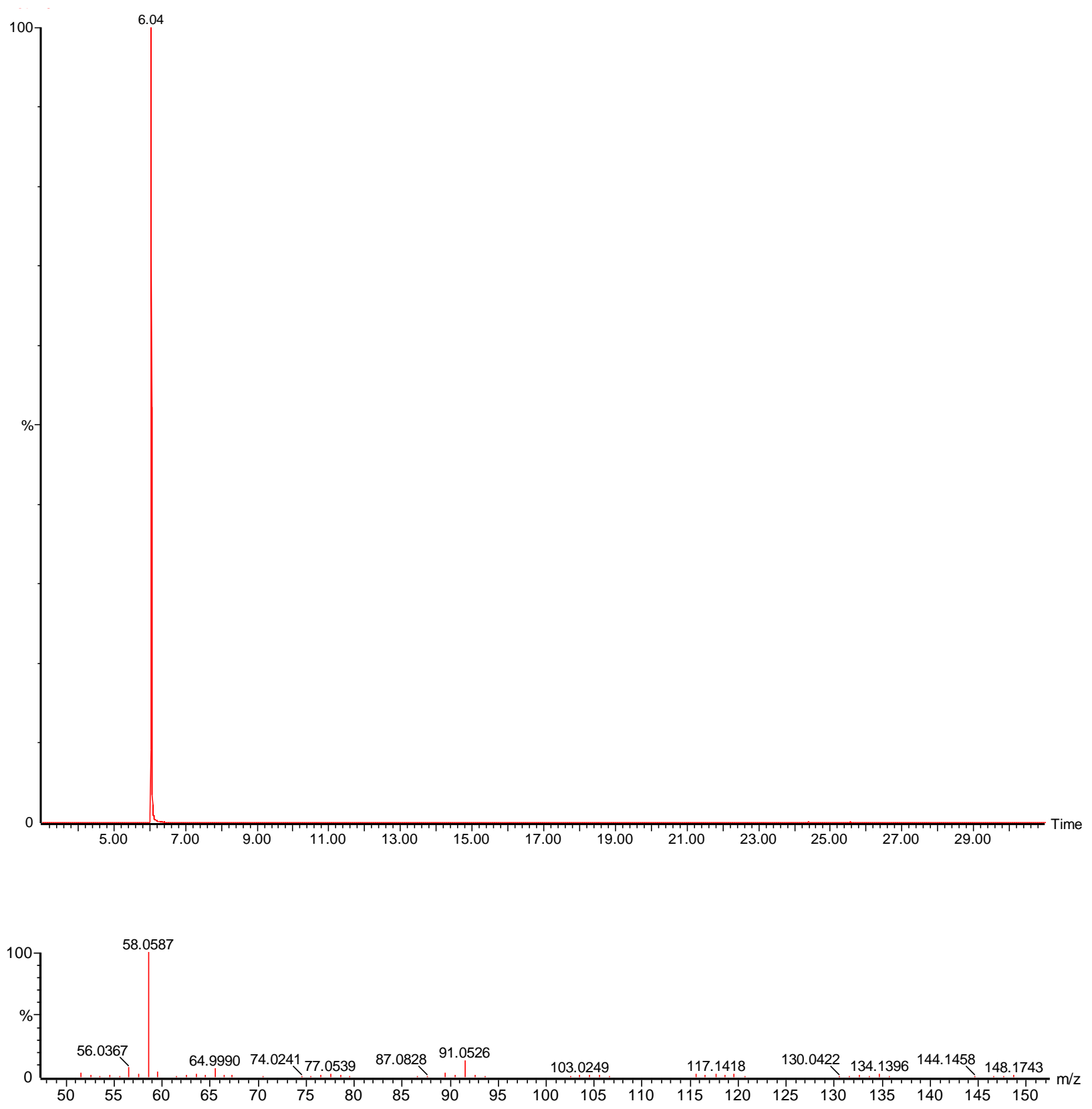


\section{Amphetamine}
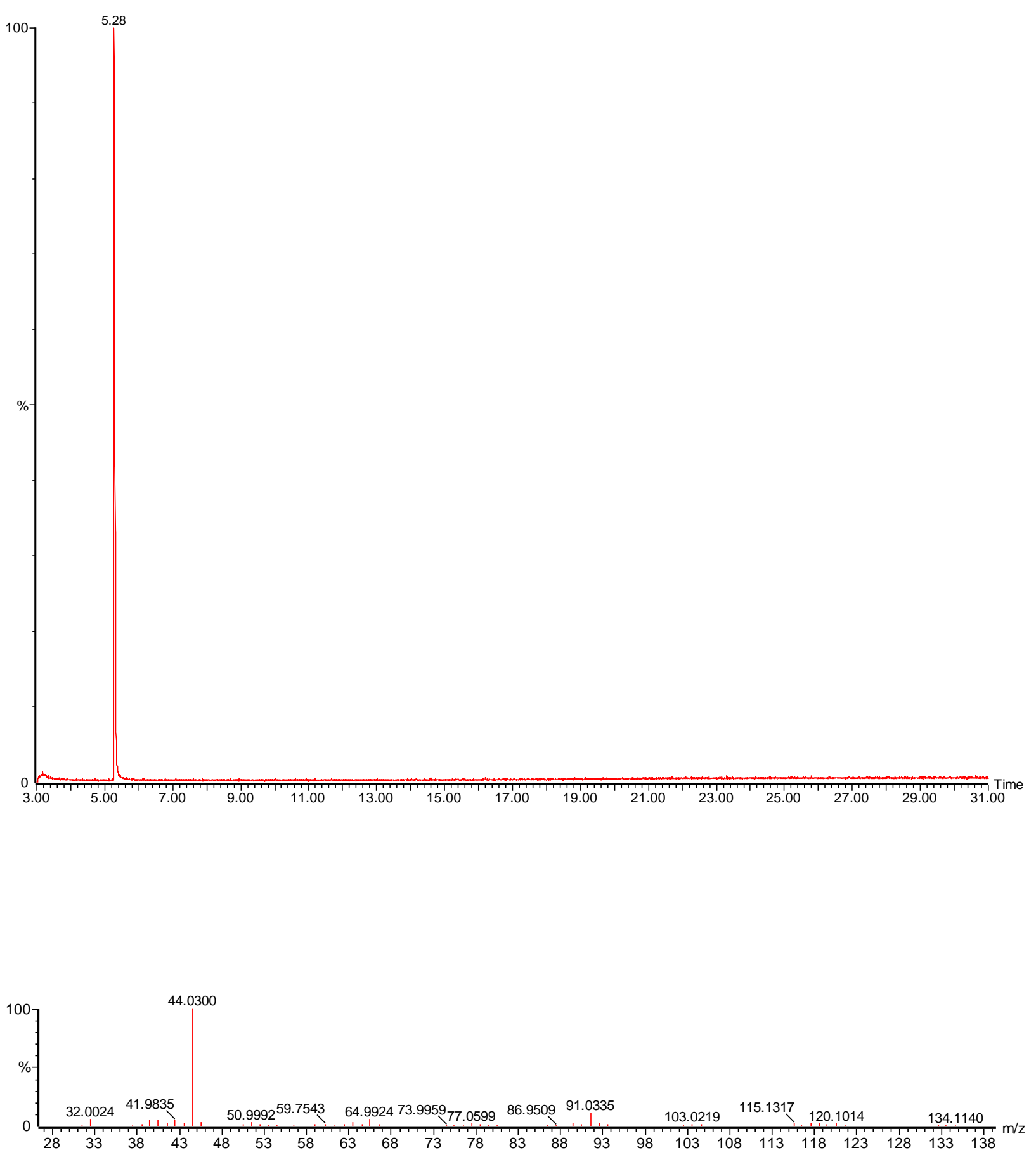


\section{Phentermine}
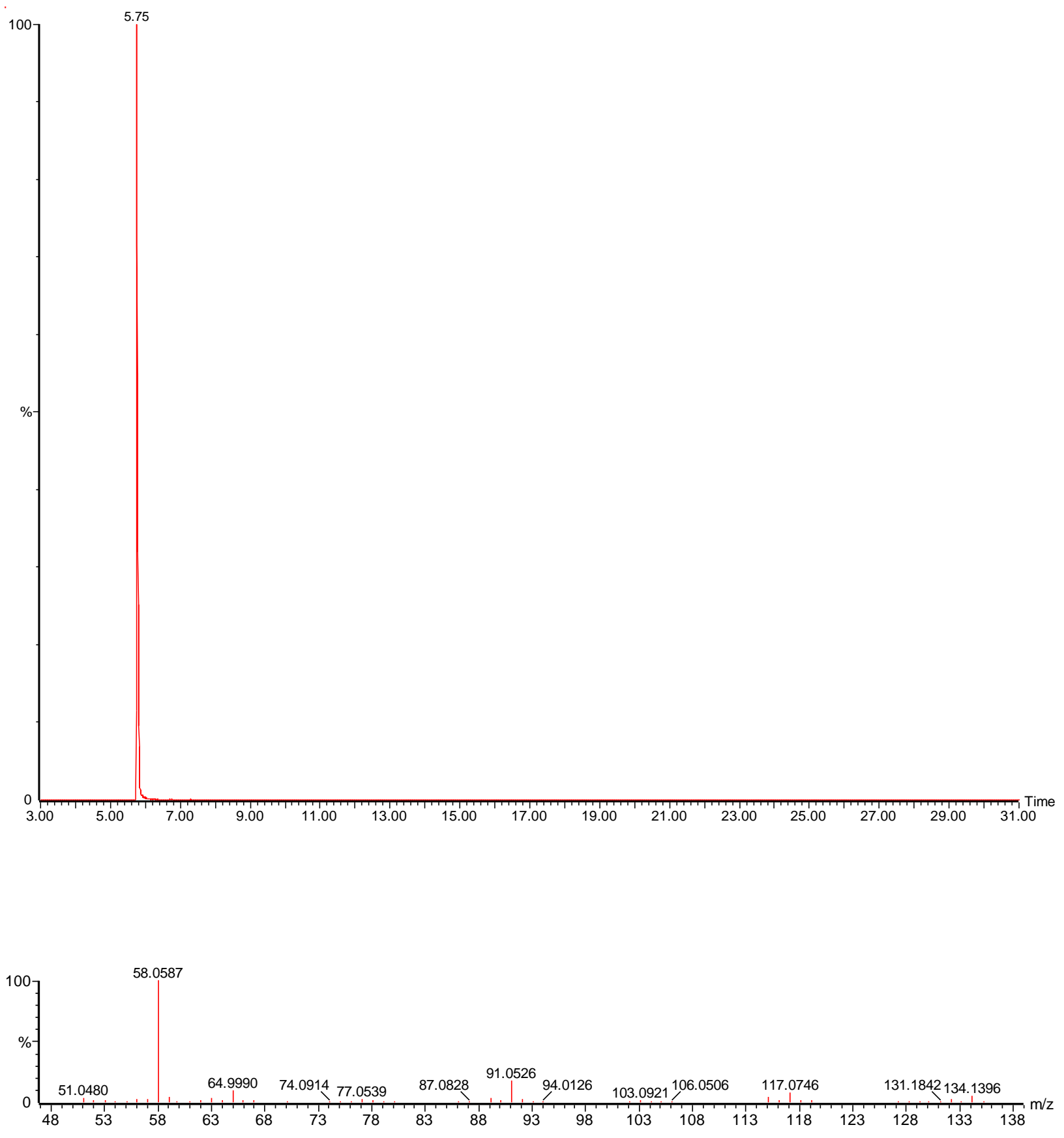


\section{Heroin}
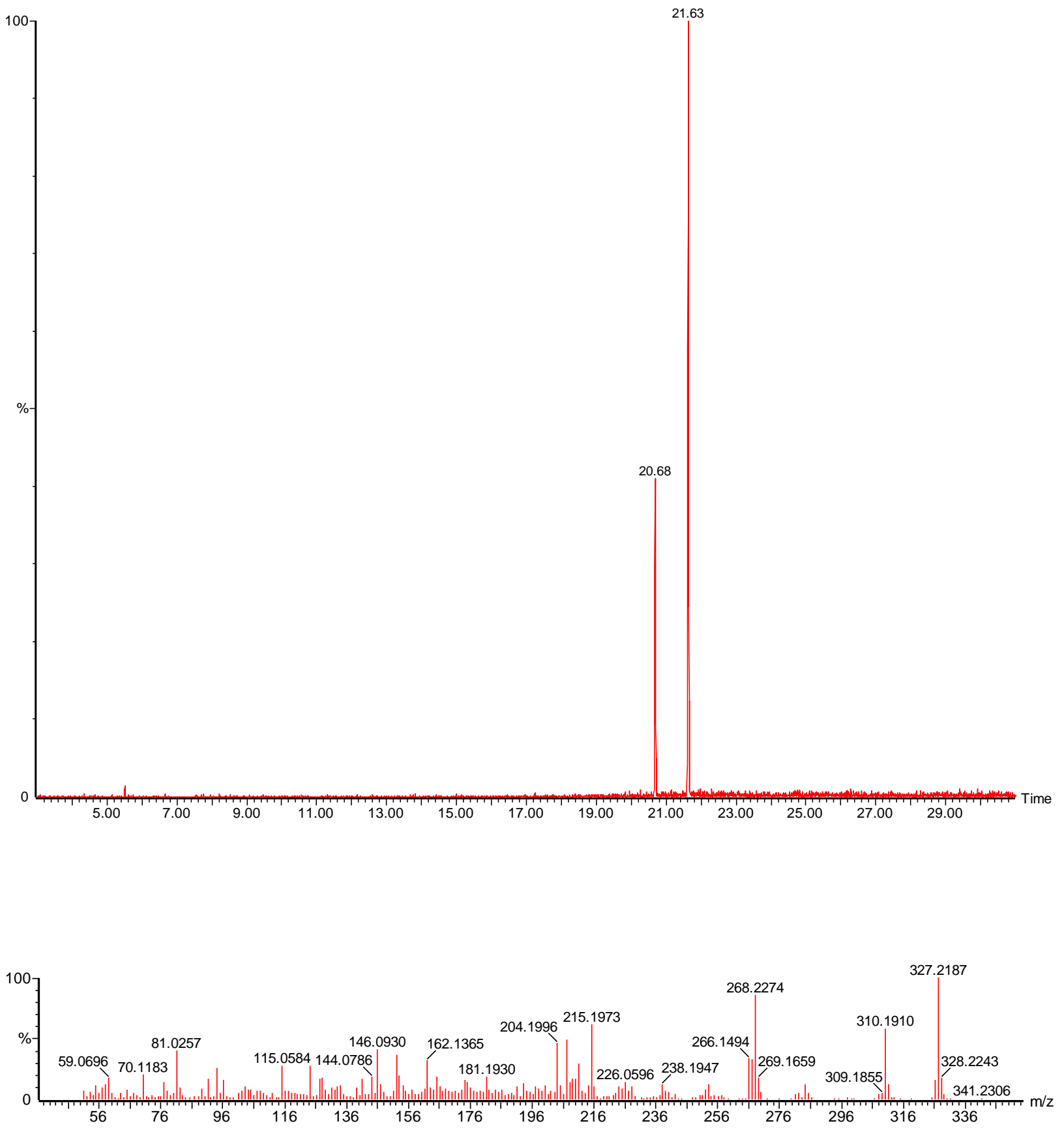


\section{Oxycodone}
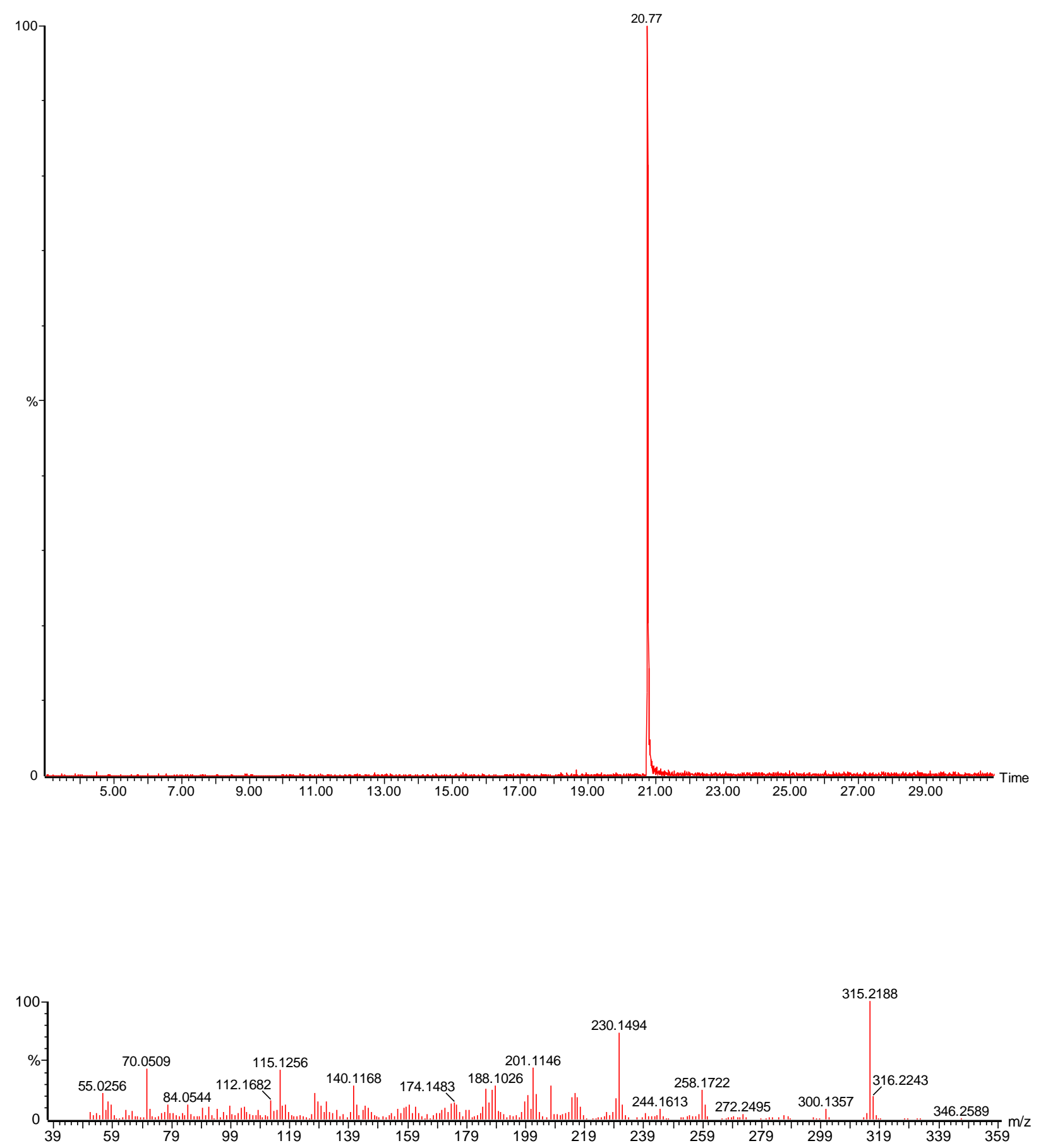


\section{Codeine}
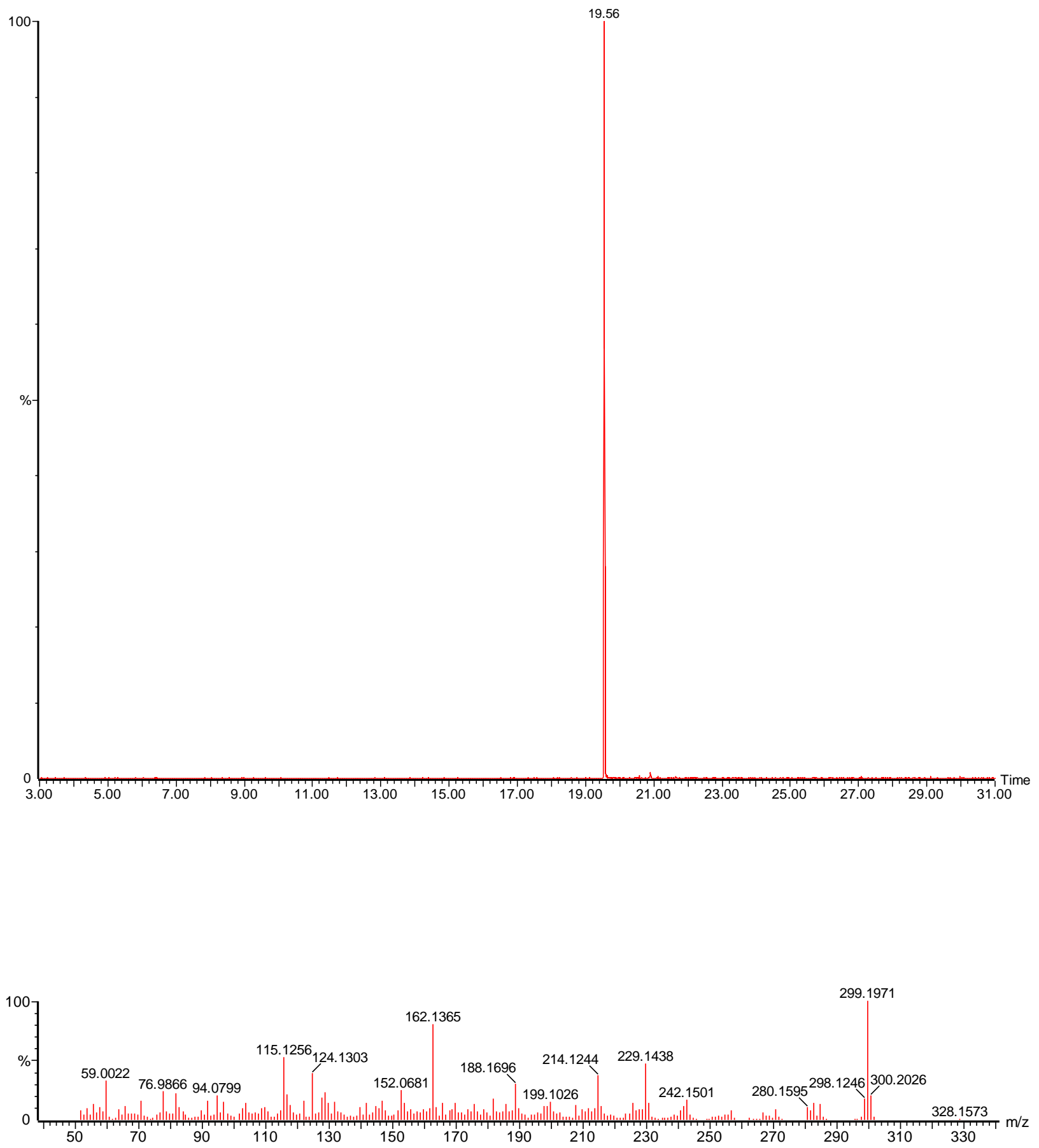


\section{Pentobarbital}
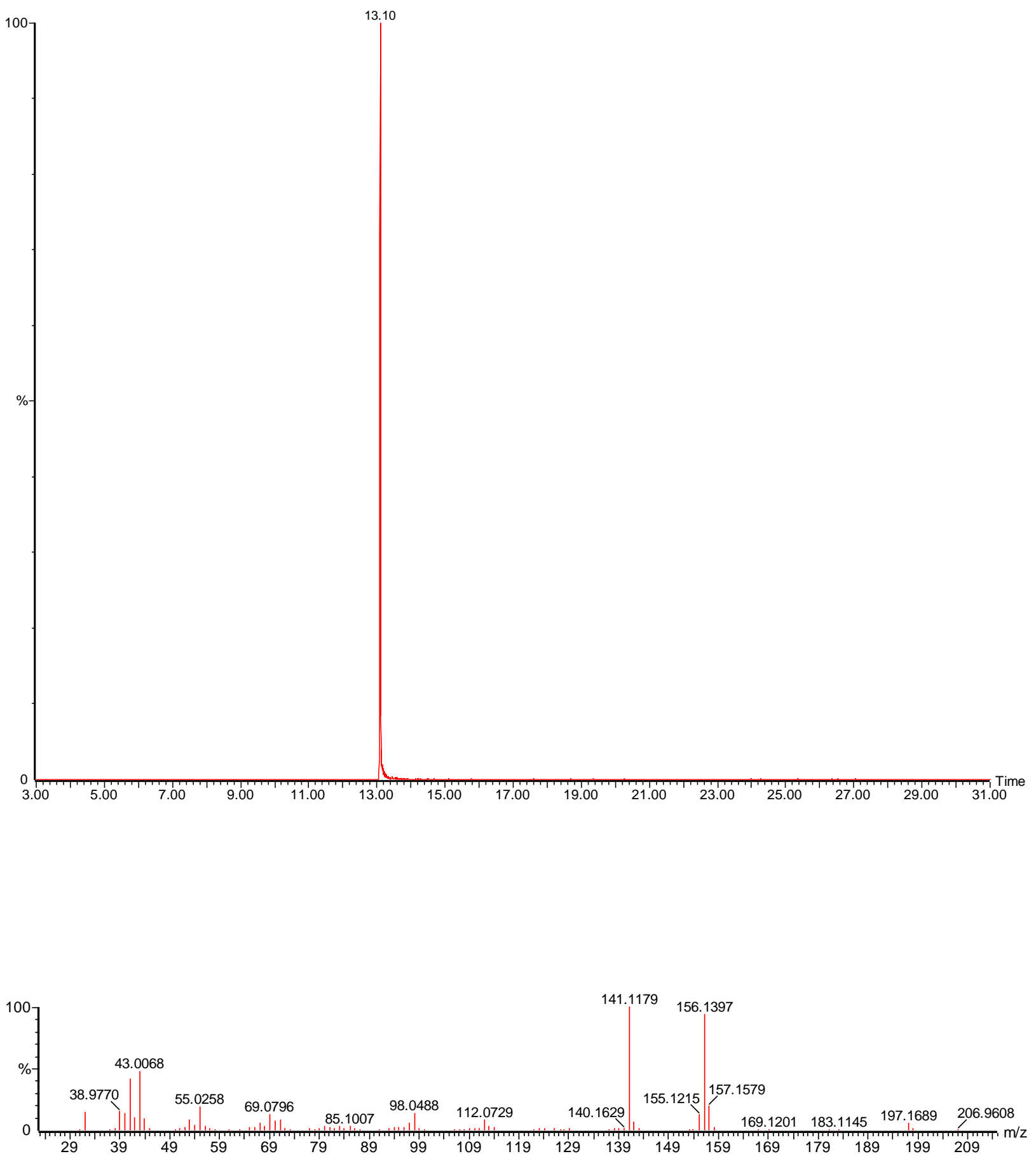


\section{Butabarbital}
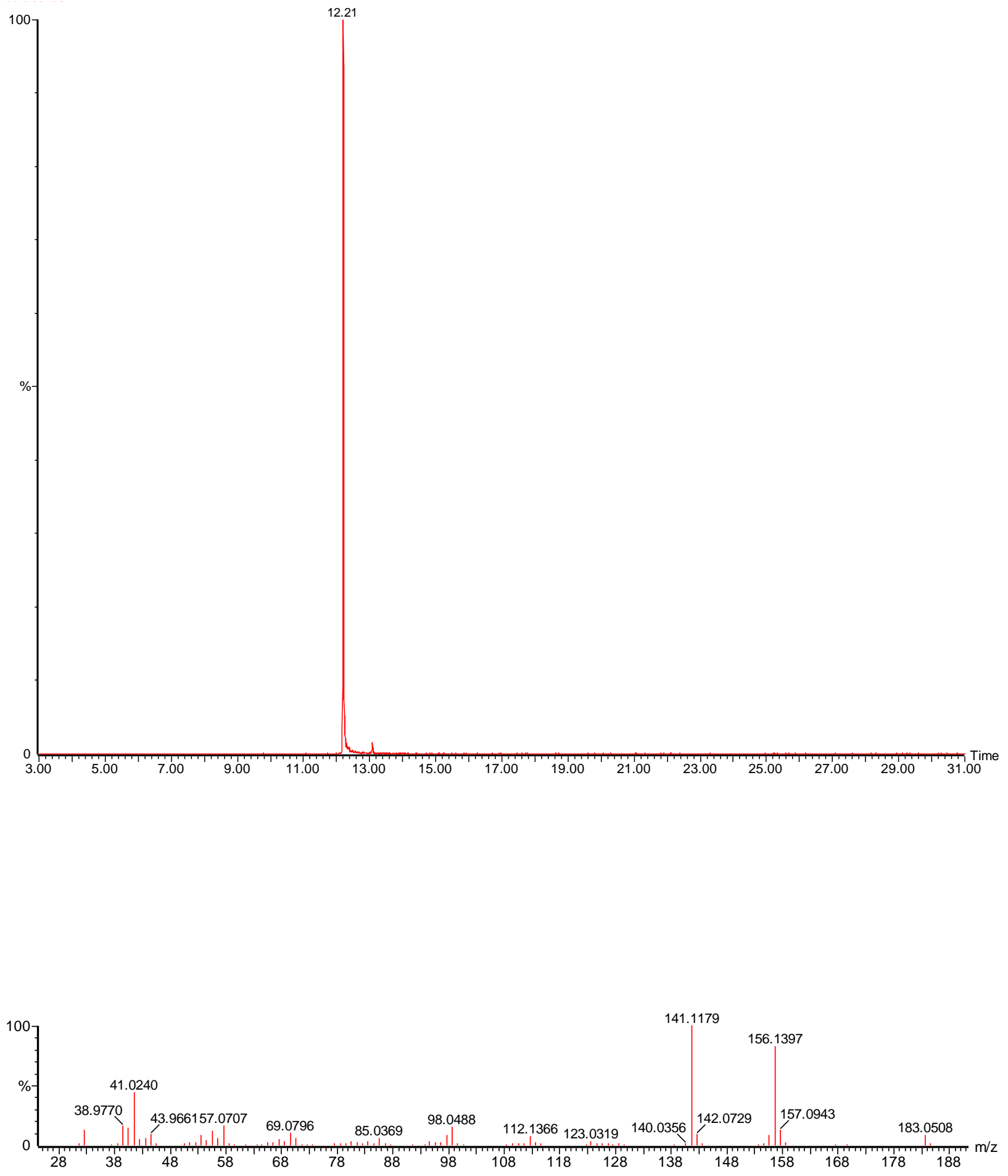


\section{Phenobarbital}
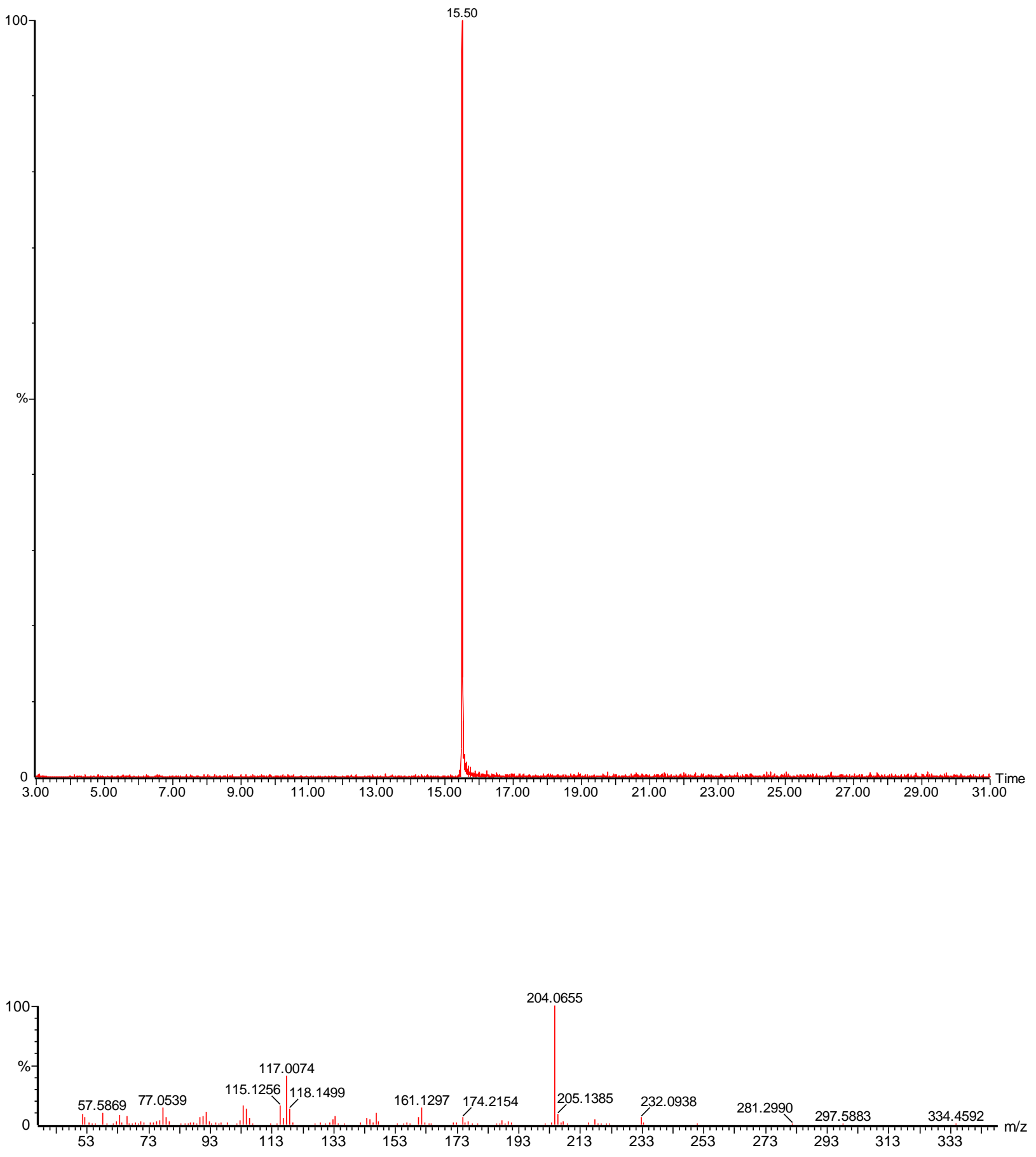